US Army Corps

of Engineers ${ }_{\circledast}$

Engineer Research and

Development Center

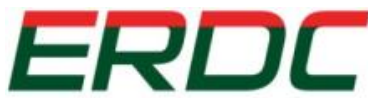

INNOVATIVE SOLUTIONS

for a safer, better world

Strategic Environmental Research and Development Program (SERDP)

\title{
Dissolution of NTO, DNAN, and Insensitive Munitions Formulations and Their Fates in Soils
}

Katerina Dontsova, Susan Taylor, Rose Pesce-Rodriguez,

September 2014

Mark Brusseau, Jennifer Arthur, Noah Mark, Marianne Walsh, James Lever, and Jiri Šimůnek
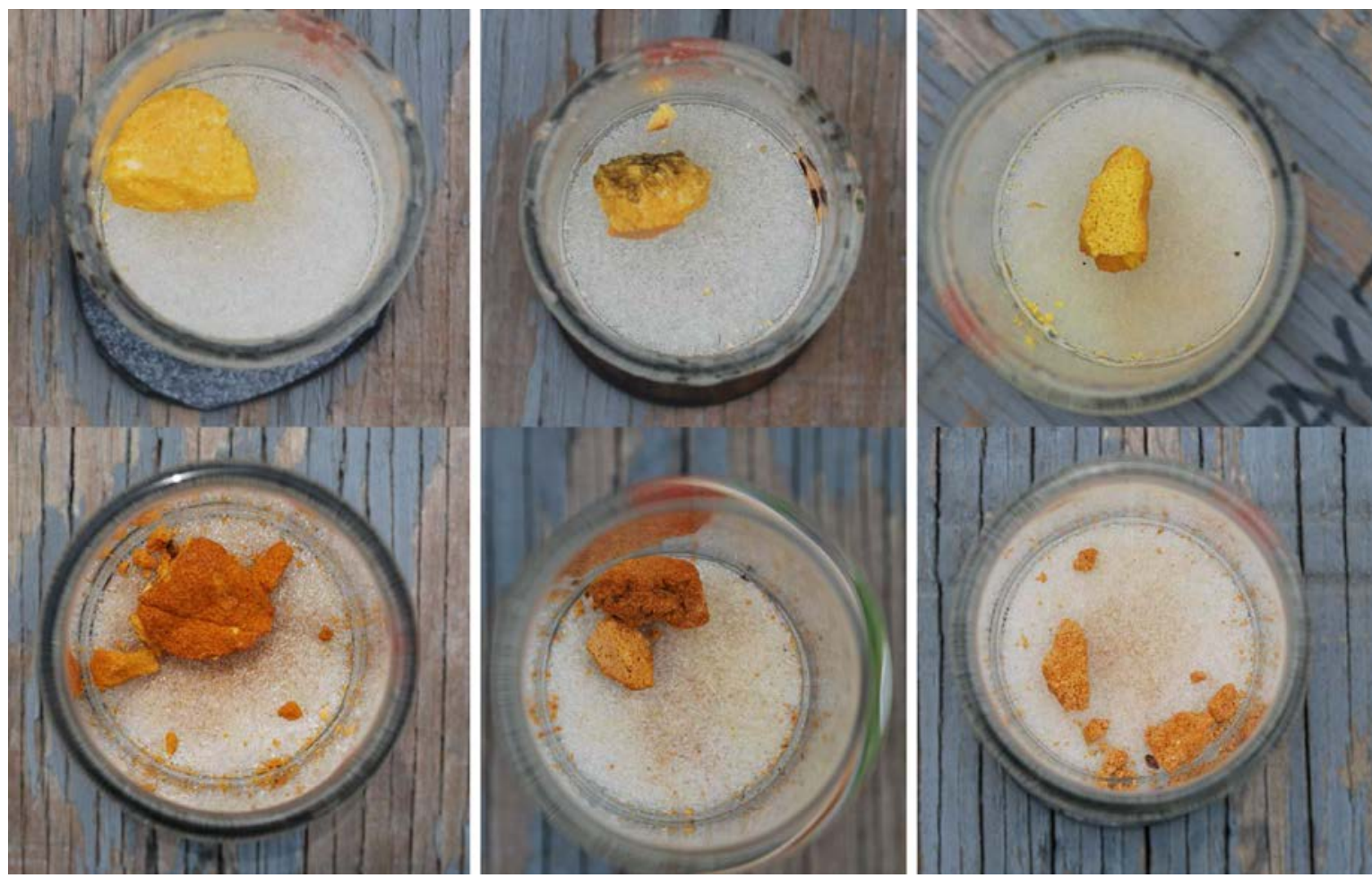

Effects of outdoor weathering and dissolution on three insensitive munitions formulations after 17 days (top) and 1 year (bottom). 
The U.S. Army Engineer Research and Development Center (ERDC) solves the nation's toughest engineering and environmental challenges. ERDC develops innovative solutions in civil and military engineering, geospatial sciences, water resources, and environmental sciences for the Army, the Department of Defense, civilian agencies, and our nation's public good. Find out more at www.erdc.usace.army.mil.

To search for other technical reports published by ERDC, visit the ERDC online library at http://acwc.sdp.sirsi.net/client/default. 


\section{Dissolution of NTO, DNAN and Insensitive Munitions Formulations and Their Fates in Soils}

Katerina Dontsova, Mark Brusseau, Jennifer Arthur, and Noah Mark

University of Arizona

845 N. Park Avenue

Tucson, AZ 85721-0158

Susan Taylor, James Lever, and Marianne Walsh

Cold Regions Research and Engineering Laboratory (CRREL)

U.S. Army Engineer Research and Development Center

72 Lyme Road

Hanover, NH 03755-1290

Rose Pesce-Rodriguez

Army Research Laboratory (ARL), Weapons and Materials Research Directorate (WMRD)

U.S. Army Research Laboratory

Aberdeen Proving Ground, MD 21005-5006

Jiri Šimůnek

University of California, Department of Environmental Sciences

Riverside, CA 925021

Final Report

Approved for public release; distribution is unlimited.

Prepared for Strategic Environmental Research and Development Program (SERDP) Alexandria, VA 22350-3605

Under ER-2220, "Dissolution of NTO, DNAN and Insensitive Munitions Formulations and their Fates in Soils" 


\section{Abstract}

New explosive compounds that are less sensitive to shock and high temperatures are being tested as replacements for TNT and RDX. Two of these explosives, DNAN (2,4-dinitroanisole) and NTO (3-nitro-1,2,4-triazol-5one), have good detonation characteristics and are the main ingredients in a suite of insensitive munitions (IM) explosives that are being, or soon will be, fielded. Both compounds, however, are more soluble than either TNT or RDX; and research has shown that both have some human and environmental toxicity. Data on their fate is needed to determine if DNAN and NTO have the potential to reach groundwater and be transported off base, an outcome that could create future contamination problems on military training ranges and trigger regulatory action. In this study, we measured how quickly DNAN and NTO dissolve in IM formulations and how solutions of these IM explosives interact with different types of soils. Because both dissolution and solution- soil interactions are determined by a suite of parameters, we are using a multifaceted approach to studying these processes. Given a mass of IM compounds scattered on the range, our work will help determine the dissolved IM masses, their subsequent transport and fate, and their likelihood of reaching groundwater.

DISCLAIMER: The contents of this report are not to be used for advertising, publication, or promotional purposes. Citation of trade names does not constitute an official endorsement or approval of the use of such commercial products. All product names and trademarks cited are the property of their respective owners. The findings of this report are not to be construed as an official Department of the Army position unless so designated by other authorized documents. 


\section{Contents}

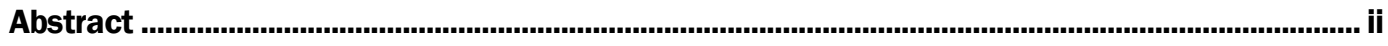

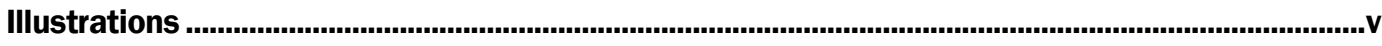

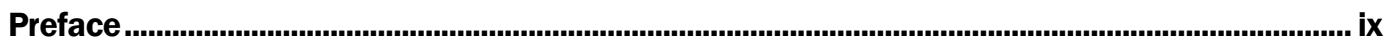

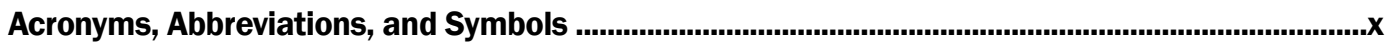

1 Introduction .................................................................................................................................. 1

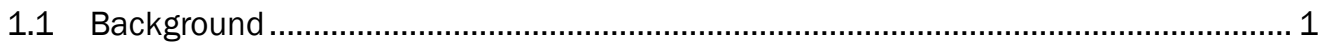

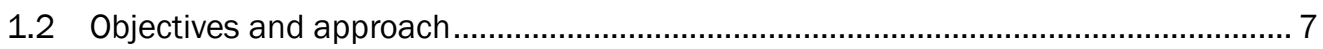

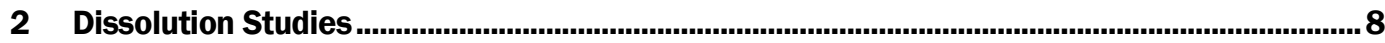

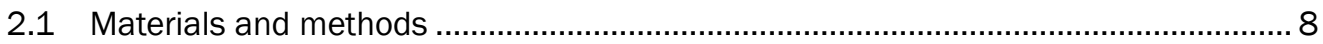

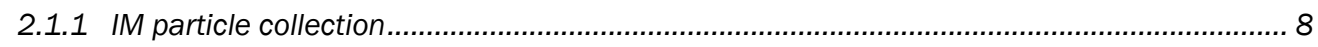

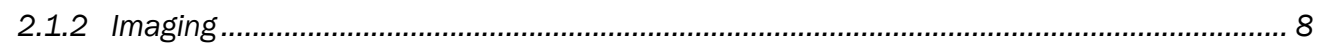

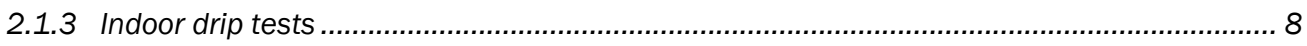

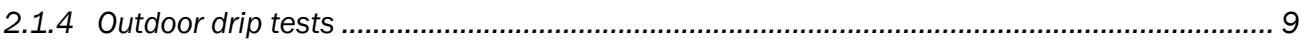

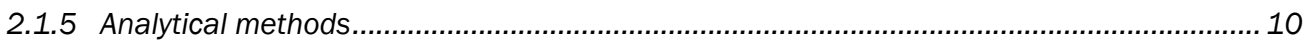

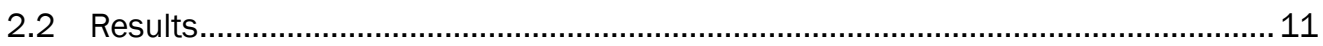

2.2.1 Characterized particles of DNAN, NTO, and detonation residues of IM

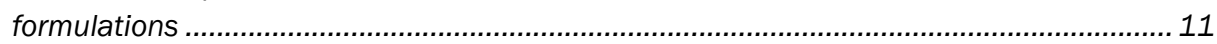

2.2.2 Laboratory tests to measure the dissolution of IM detonation residues .............................15

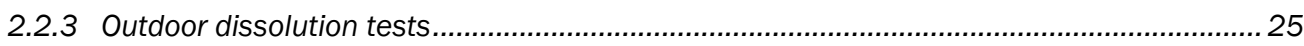

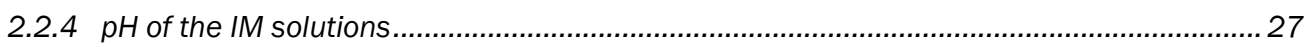

3 Phototransformation studies...........................................................................................29

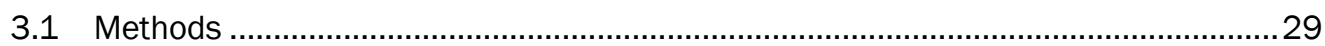

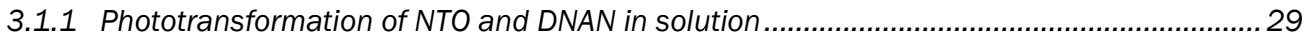

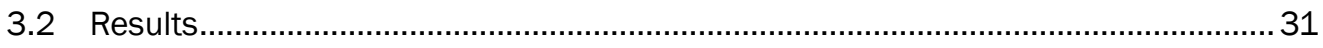

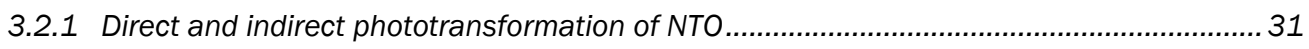

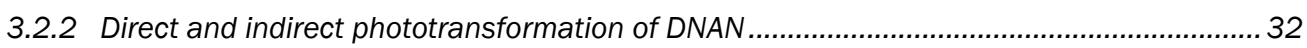

3.2.3 Phototransformation of NTO and DNAN as a function of temperature................................ 34

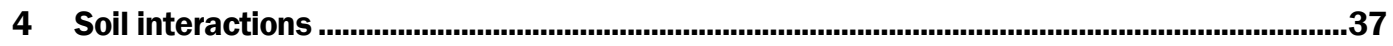

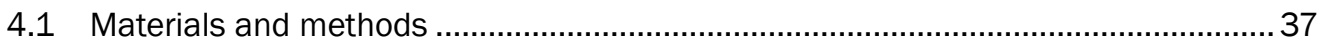

4.1.1 Soil sample collection and characterization ....................................................................... 37

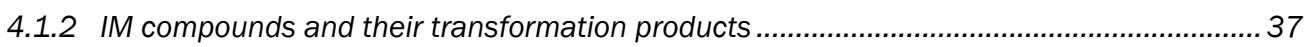

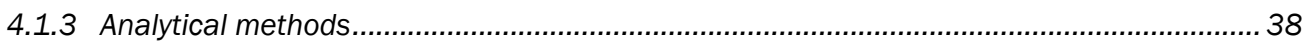

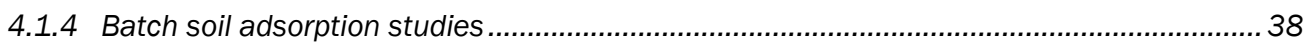

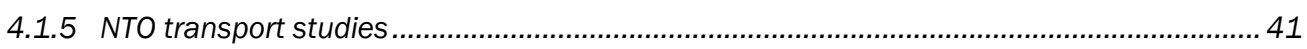

4.1.6 IM formulation dissolution and transport studies ...........................................................42

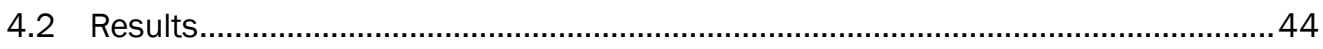


4.2.1 Soil sample collection and characterization .................................................................. 44

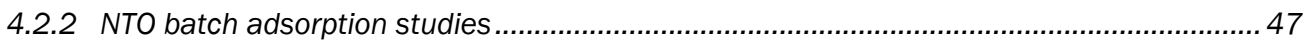

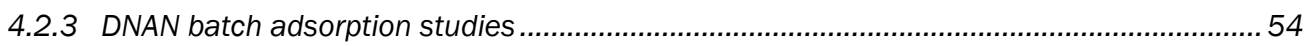

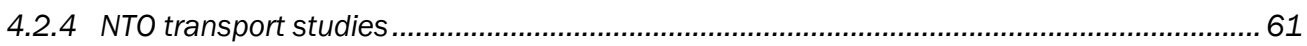

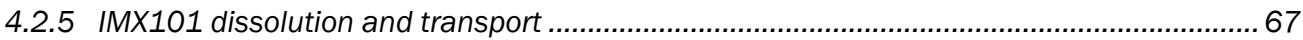

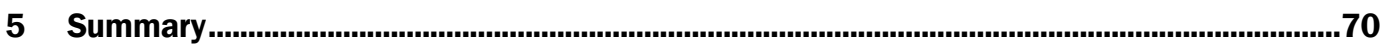

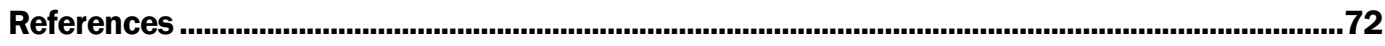

Report Documentation Page 


\section{Illustrations}

\section{Figures}

1 Plots of the solubility of (a) DNAN and (b) NTO as a function of temperature. Data are from studies conducted at the U.S. Army Construction Engineering Research Laboratory (CERL) (Boddu et al. 2008), the Army Research Laboratory (ARL) (R. Pesce-Rodriguez, unpublished), the Defense Science and Technology Organization (Smith and Cliff 1999), and others.

2 Mass loss measured by high precision liquid chromatography (HPLC) versus measured mass loss by electronic balance for TNT and Tritonal (a TNTaluminum explosive) samples (Taylor et al. 2010)

3 Photos of (a) the laboratory drip tests and of (b) the outdoor setup.....................................

4 Optical images of DNAN, NTO, IMX101, IMX104, and PAX21 and the compositions of the three IM formulations we studied.

5 Images of (a) the Comp B surface and of (b) an IMX104 particle. These images show that as the TNT matrix dissolves from Comp B, the less soluble RDX crystals jut from the surface and eventually fall off the particle. For IMX104, on the other hand, the DNAN matrix is less soluble than the crystal constituents and dissolution produces hole-riddled particles.

6 PAX21 particles: (a) detonation residue; (b) AP completely dissolved; (c) DNAN almost completely dissolved; and (d) RDX crystals remaining.

7 Shown are radiographs and reconstructed cross sections of IMX101 ( $a$ and $b$ ), IMX104 (c and d), and PAX21 (e and f). Also shown is the same PAX21 particle after a half-hour submersion in water ( $g$ and $h$ ). The scale bar applies to only the cross sections. The diameter of the post used to mount the samples for the radiographs was $3 \mathrm{~mm}$

8 Images of undetonated, detonated, and wetted IM particles. Note the fractures in the detonated particles and the loss of components in the wetted particles

9 Cumulative mass dissolved in the water dripped on the $\mu$ CT-imaged (a) IMX101, (b) IMX104, and (c) PAX21 particles.

10 Change in the cross section of each formulation with increased water interaction ...........18

11 Measurements made possible by $\mu \mathrm{CT}$ : (a) volume of particles and crystal constituents in IM particles and (b) Density calculated from the mass and $\mu \mathrm{CT}$ volume measurements.

12 Percent of the expected mass dissolved for compounds in IMX101 versus water volume $(\mathrm{mL})$

13 Percent of the expected mass dissolved for compounds in IMX104 versus water

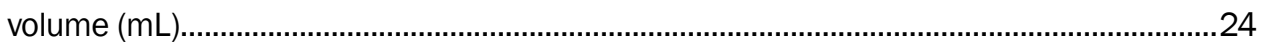

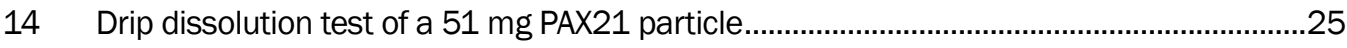

15 Percent mass dissolved of the mass expected for the compounds in 5 chunks of IMX101, 5 chunks of IMX104, and 2 chunks of PAX21 set outside to weather and dissolve 
17 Plot showing the relationship between NTO concentration and $\mathrm{pH}$ for indoor drip samples and outdoor samples. These data look similar to a titration curve.

18 SUNTEST CPS + Xenon Test Instrument used to evaluate phototransformation of NTO and DNAN in solution ...................................................................................................29

19 Ionized and neutral forms of NTO (Smith and Cliff 1999) ...............................................30

20 Phototransformation of NTO as affected by (a) solution pH and (b) the presence of natural organic matter.

21 Phototransformation rate of DNAN in water and in water with humic acid. Error bars are smaller than the symbols

22 Effect of temperature on DNAN and NTO phototransformation rates ..

23 Natural logarithm of the phototransformation rate constant plotted as a function of temperature for DNAN and NTO

24 Measured and predicted values of NTO and DNAN half-lives as a function of temperature

25 Experimental setup for the IM column transport studies

26 Map of the continental Unites States indicating location of soil collection. 46

27 Plot of (a) NTO solution concentrations normalized by input over time in the presence of Plymouth soil and (b) the same concentrations plotted as the natural logarithm of concentrations in order to determine the transformation rate constant of NTO. Error bars equal one standard error of the mean $(n=9$ with 3 independent replicate test tubes and 3 repeat HPLC measurements for each sample).

28 Correlation between measured NTO transformation rate constants $(k)$ and soil OC. $\mathrm{P}=0.02250$

29 Adsorption isotherms calculated for NTO in Catlin, Fort Harrison, and Limestone Hills soils. The red dashed line indicates the linear adsorption isotherm fit to the measured adsorption data (equation is in red) while the solid black line is a fit of the Freundlich isotherm with the equation presented in black

30 Adsorption isotherms calculated for NTO in Sassafras, Plymouth, and Camp Gruber soils. The red dashed line indicates the linear adsorption isotherm fit to the measured adsorption data (equation is in red) while the solid black line is a fit of the Freundlich isotherm with the equation presented in black

31 Adsorption isotherms calculated for NTO in Camp Butner, Florence MR, and Camp Guernsey soils. The red dashed line indicates the linear adsorption isotherm fit to the measured adsorption data (equation is in red) while the solid black line is a fit of the Freundlich isotherm with the equation presented in black

32 Adsorption isotherms calculated for NTO in Arnold AFB and Camp Swift soils. The red dashed line indicates the linear adsorption isotherm fit to the measured adsorption data (equation is in red) while the solid black line is a fit of the Freundlich isotherm with the equation presented in black

33 Measured NTO adsorption coefficients $\left(K_{d}\right)$ plotted against soil pH. P $=0.00011$

34 Plot of (a) normalized DNAN solution concentrations in contact with Camp Guernsey soil and (b) the same concentrations plotted as the natural logarithm whose slope gives the transformation rate, $k$, of NTO.

35 Adsorption isotherms calculated for DNAN in Arnold AFB, Camp Swift, and Sassafras soils. The red dashed line indicates the linear adsorption isotherm fit to the measured adsorption data (equation is in red) while the solid black line is a fit of the Freundlich isotherm with the equation presented in black 
36 Adsorption isotherms calculated for DNAN in Catlin, Florence MR, and Camp Gruber soils. The red dashed line indicates the linear adsorption isotherm fit to the measured adsorption data (equation is in red) while the solid black line is a fit of the Freundlich isotherm with the equation presented in black

37 Adsorption isotherms calculated for DNAN in Camp Guernsey, Camp Butner, and Plymouth soils. The red dashed line indicates the linear adsorption isotherm fit to the measured adsorption data (equation is in red) while the solid black line is a fit of the Freundlich isotherm with the equation presented in black

38 Adsorption isotherms calculated for DNAN in Limestone Hills and Fort Harrison soils. The red dashed line indicates the linear adsorption isotherm fit to the measured adsorption data (equation is in red) while the solid black line is a fit of the Freundlich isotherm with the equation presented in black

39 Correlation between (a) measured DNAN linear $\left(K_{d}\right)$ and (b) Freundlich $\left(K_{f}\right)$ adsorption coefficients and soil percent OC. $P=0.00061$ for $K_{d}$ and 0.00011 for $K_{f}$, both highly significant relationships

40 Breakthrough curves for NTO in Camp Swift soil. The dashed grey vertical line indicates timing of $24 \mathrm{hr}$ flow interruption. The tan vertical line indicates the time when the solution was changed back to a saturating solution to observe the desorption phase of the isotherm.

41 Breakthrough curves for NTO in Camp Guernsey soil. The dashed grey vertical line indicates timing of $24 \mathrm{hr}$ flow interruption. The tan vertical line indicates the time when the solution was changed back to a saturating solution to observe the desorption phase of the isotherm.

42 Breakthrough curves for NTO in Sassafras soil. The dashed grey vertical line indicates timing of $24 \mathrm{hr}$ flow interruption. The tan vertical line indicates the time when the solution was changed back to a saturating solution to observe the desorption phase of the isotherm.

43 Breakthrough curves for NTO in Arnold AFB soil. The dashed grey vertical line indicates timing of $24 \mathrm{hr}$ flow interruption. The tan vertical line indicates the time when the solution was changed back to a saturating solution to observe the desorption phase of the isotherm.

44 Correlation between batch and column NTO linear adsorption coefficients $\left(K_{d}\right)$. $\mathrm{P}=0.00185$ for $K_{d}$, which is highly significant.

45 Breakthrough curves for NTO; NQ; DNAN; and DNAN transformation product, MENA, from IMX101 particle dissolution in Camp Swift soil: (a) undetonated particles and (b) residues of low order detonation. The flow rate is $0.01 \mathrm{~mL}$ $\mathrm{min}^{-1}$. The black vertical line marks the time when the IMX101 particles were removed from the soil surface to observe desorption phase of the breakthrough

46 Breakthrough curves for NTO; NQ; DNAN; and DNAN transformation product, MENA, from undetonated IMX101 particle dissolution in Camp Guernsey soil. Flow rate is $0.01 \mathrm{~mL} \mathrm{~min}^{-1}$. The black vertical line marks the time when the IMX101 particle was removed from the soil surface to observe the desorption phase of the breakthrough

\section{Tables}

1 Selected environmentally relevant chemical and physical properties of DNAN, NTO, TNT, and RDX, including solubility at $25^{\circ} \mathrm{C}$, octanol-water partition coefficient $\left(K_{o w}\right)$, and soil organic carbon adsorption coefficient $\left(K_{o c}\right)$ 
2 IM formulations containing DNAN and NTO in advanced stages of development

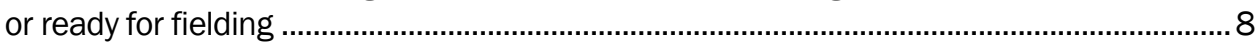

3 Mass balance for IMX101, IMX104, and PAX21 particles used in the $\mu$ CT drip dissolution tests. Also tabulated are the calculated volumes for each particle.*

4 Mass balance for IMX101 and IMX104 drip dissolution tests. The expected mass was calculated based on the initial mass of the particle and the putative compositions of the formulations ..

5 Determined phototransformation rate constants, $k_{\text {photo, }}$, and half-lives, $t \frac{1}{2}$, , for NTO and DNAN measured at $35^{\circ} \mathrm{C}, 765$ Watts $/ \mathrm{m}^{2}$

6 Phototransformation rate constant, $k_{\text {photo, }}$, and half-life, $t_{1} / 2$, of NTO and DNAN tabulated as a function of temperature. Measurements were made at $765 \mathrm{~W} / \mathrm{m}^{2}$ irradiance.

7 Soil:solution ratios tested in NTO and DNAN soil adsorption studies

8 Soils used to study interaction of IMs with the soils. Series names and classifications are according to U.S. Taxonomy and location where collected

9 Measured physical and chemical properties of soils used in adsorption and transport studies with NTO, DNAN, and IM formulations

10 Fate and transport parameters for NTO in studied soils: Freundlich adsorption parameters, $K_{f}$ and $n$; soil adsorption coefficient, $K_{d}$; adsorption coefficient normalized to fraction of organic carbon in soils, Koc; transformation rate constant, $k$; and $R^{2}$ values for the fits

11 Fate and transport parameters for DNAN in studies soils: Freundlich adsorption parameters, $K_{f}$ and $n$; linear adsorption coefficient, $K_{d}$; adsorption coefficient normalized to fraction of organic carbon in soils, Koc; transformation rate constant, $k$; and $R^{2}$ values for the fits.

12 Estimates of fate and transport parameters; linear adsorption coefficients, $K_{d ;}$ and transformation rate constants, $k$, for NTO determined from batch and column experiments 


\section{Preface}

This study was conducted for the Environmental Restoration Program under Strategic Environmental Research and Development Program (SERDP) ER-2220, "Dissolution of NTO, DNAN and Insensitive Munitions Formulations and their Fates in Soils." The technical monitor was Dr. Andrea Leeson, SERDP.

The work was performed by Dr. Susan Taylor and Marianne Walsh (Biogeochemical Sciences Branch, Timothy Cary, Chief) and Dr. J ames Lever (Force Projection and Sustainment Branch, Dr. Edel Cortez, Chief), U.S. Army Engineer Research and Development Center, Cold Regions Research and Engineering Laboratory (ERDC-CRREL); Dr. Katerina Dontsova, Dr. Mark Brusseau, J ennifer Arthur, and Noah Mark, University of Arizona; Dr. Rose Pesce-Rodriguez, Army Research Laboratory (ARL), Weapons and Materials Research Directorate (WMRD); and Dr. Jiri Šimůnek, University of California, Department of Environmental Sciences. At the time of publication, Dr. J ustin Berman was Chief of the Research and Engineering Division. The Deputy Director of ERDC-CRREL was Dr. Lance Hansen, and the Director was Dr. Robert Davis.

We thank Dr. Andrea Leeson, Dr. Anne Andrews, and Dr. J effrey Marqusee for their support. In addition, we would like to acknowledge Anthony Di Stasio and Erika Rivera, U.S. Army Armament Research, Development and Engineering Center (ARDEC), Picatinny Arsenal, for providing NTO, DNAN, IMX101, IMX104, and PAX21 IM formulations; we also appreciate Dr. Michael Walsh and project SERDP ER-2219 for providing detonation residues from tests with IM formulations. We are extremely grateful to Bonnie M. Packer from the Army National Guard Environmental Directorate and Rosa Gwinn, Lisa DeGrazia, Bethany Keller, J essica Milose, Amibeth Sheridan, Laurie Stenberg, and Sarah Gettier from the URS Corporation, Germantown, MD, who collected soil samples at the National Guard sites. We are thankful to Mike Heitmann (CH2M HILL Englewood, CO) for collecting soil at Camp Butner, NC, and at Florence Military Reservation, AZ.

The Commander of ERDC was COL J effrey R. Eckstein, and the Director of ERDC was Dr. J effery P. Holland. 


\section{Acronyms, Abbreviations, and Symbols}

\begin{tabular}{|c|c|}
\hline$\mu \mathrm{CT}$ & Micro-computed Tomography \\
\hline 3-D & Three-Dimensional \\
\hline $\mathrm{AcN}$ & Acetonitrile \\
\hline $\mathrm{AFB}$ & Air Force Base \\
\hline $\mathrm{AP}$ & Ammonium Perchlorate \\
\hline ARDEC & Armament Research, Development and Engineering Center \\
\hline ARL & Army Research Laboratory \\
\hline ATO & 5-amino-1,2,4-triazol-3-one \\
\hline $\mathrm{Br}^{-}$ & Bromide Tracer \\
\hline $\mathrm{C}$ & Contaminant Concentration in Solution \\
\hline $\mathrm{C}_{0}$ & Initial Concentration of Target Analyte in Solution \\
\hline $\mathrm{C} 4$ & $\begin{array}{l}\text { Composition 4, a common variety of the plastic explosive known as } \\
\text { "C4" }\end{array}$ \\
\hline $\mathrm{CaCl}_{2}$ & Calcium Chloride \\
\hline $\mathrm{CE}$ & 95\% Confidence Interval \\
\hline CERL & Construction Engineering Research Laboratory \\
\hline Comp B & $\begin{array}{l}\text { Composition B, a 39:60 mix of TNT and RDX that contains about } \\
1 \% \text { wax }\end{array}$ \\
\hline CRREL & Cold Regions Research and Engineering Laboratory \\
\hline DNAN & 2,4-dinitroanisole \\
\hline DNT & 2,4- or 2,6-dinitrotoluene \\
\hline $\mathrm{E}_{\mathrm{a}}$ & Activation Energy \\
\hline $\mathrm{EC}$ & Electrical Conductivity \\
\hline
\end{tabular}




\begin{tabular}{|c|c|}
\hline $\mathrm{E}_{\mathrm{m}}$ & One-electron standard reduction potential \\
\hline ERDC & U.S. Army Engineer Research and Development Center \\
\hline $\mathrm{H}_{3} \mathrm{PO}_{4}$ & Phosphoric Acid \\
\hline HMX & $\begin{array}{l}\text { High Explosive 1,3,5,7,-octahydro-1,3,5,7-tetranitrotetrazocine } \\
\text { (often a bi-product of RDX production) }\end{array}$ \\
\hline HPLC & High Precision Liquid Chromatography \\
\hline IM & Insensitive Munitions \\
\hline IMX & Insensitive Munitions eXplosive \\
\hline $\mathrm{k}$ & First-Order Transformation Rate Constant \\
\hline $\mathrm{k}_{\text {photo }}$ & First-Order Phototransformation Rate Constant \\
\hline $\mathrm{K}_{\mathrm{d}}$ & Linear Soil Adsorption Coefficient \\
\hline $\mathrm{K}_{\mathrm{f}}$ & Freundlich Soil Adsorption Coefficient \\
\hline KoC & Soil Organic Carbon Adsorption Coefficient \\
\hline $\mathrm{K}_{\mathrm{ow}}$ & Octanol- Water Partition Coefficient \\
\hline MENA & 2-methoxy-5-nitroaniline \\
\hline MR & Military Reservation \\
\hline $\mathrm{n}$ & A parameter in Freundlich adsorption isotherm \\
\hline $\mathrm{NaCl}$ & Sodium Chloride \\
\hline NQ & Nitroguanidine \\
\hline NTO & 3-nitro-1,2,4-triazol-5-one \\
\hline OC & Organic Carbon \\
\hline $\mathrm{OM}$ & Organic Matter \\
\hline $\mathrm{P}$ & Probability \\
\hline PAX & Picatinny Arsenal eXplosive \\
\hline
\end{tabular}




$\begin{array}{ll}\mathrm{pK}_{\mathrm{a}} & \text { Acid Dissociation Constant } \\ \text { PTFE } & \text { Polytetrafluoroethylene } \\ \mathrm{R}^{2} & \text { Coefficient of Determination } \\ \mathrm{RDX} & \text { 1,3,5-hexahydro-1,3,5-trinitro-1,3,5-triazine } \\ \mathrm{S} & \text { Contaminant Concentration in Soil } \\ \text { SERDP } & \text { Strategic Environmental Research and Development Program } \\ \mathrm{SSA} & \text { Specific Surface Area } \\ \mathrm{T} & \text { Temperature } \\ \mathrm{t} & \text { Time } \\ \mathrm{t} 1 / 2 & \text { Half-life } \\ \text { TFA } & \text { Trifluoroacetic Acid } \\ \text { TNT } & \text { 2,4,6-trinitrotoluene } \\ \text { UHPLC } & \text { Ultra High Performance Liquid Chromatography } \\ \text { W.S. EPA } & \text { Whited States Environmental Protection Agency } \\ \end{array}$




\section{Introduction}

\subsection{Background}

Transforming solid high explosives, scattered onto range soils as a result of incomplete detonations, into solutions of explosive components that reach groundwater involves a series of steps. These steps include dissolution, phototransformation (both of the solid and of the aqueous solutions formed by precipitation interacting with the solid), and complex interactions of the dissolved explosives with soil constituents during transport from the surface to the groundwater. If these compounds and their degradation products are innocuous, their transport to groundwater might not matter. Unfortunately, toxicology data for DNAN (2,4-dinitroanisole) and NTO (3-nitro-1,2,4-triazol-5-one) show that while NTO has low mammalian toxicity (London and Smith 1985), DNAN is more toxic to mammals than TNT (2,4,6-trinitrotoluene) (Davies and Provatas 2006), can inhibit seed germination and plant growth, and even is used as a pesticide (Dumitras-Hutanu et al. 2009). Dodard et al. (2013) also showed that it is toxic to bacteria and earthworms. Furthermore, both DNAN and NTO can form toxic transformation products (Le Campion et al. 1999b; Davies and Provatas 2006).

DNAN and NTO are readily soluble in water, and their solubility is considerably higher than those of TNT and RDX (1,3,5-hexahydro-1,3,5-trinitro1,3,5-triazine) (Table 1; Figure 1). When these compounds are part of a formulation, their dissolution will depend not only on their individual solubility and dissolution rates but also on the fraction of each component exposed to water (Lever et al. 2005; Dontsova et al. 2006; Taylor et al. 2009a, 2009b). In Composition B (Comp B-60\% RDX, 39\% TNT, 1\% wax), for example, the more soluble TNT dissolves from the surface, exposing progressively more of the slowly dissolving RDX crystals (Lever et al. 2005). Like Comp B, the insensitive munitions (IM) formulations are produced by melt casting where one or more explosive fillers (such as AP [ammonium perchlorate], RDX, NTO, or NQ [nitroguanidine]) are mixed with a melted energetic binder (TNT or DNAN) (Pelletier et al. 2010; Rutkowski et al. 2010). However, in IMs, the filler, NTO, has a higher solubility than the binder, DNAN. Depending on the amount of each compound in the formulation, an initial loss of NTO could be followed by dissolution of both components; or DNAN may protect NTO from dissolving. 
Solubility, along with particle size and climatic conditions (rainfall and temperature), controls the dissolution rate of solid explosives (Lever et al. 2005; Taylor et al. 2009a, 2009b). Our aim is to parameterize what controls dissolution of IM formulations.

Table 1. Selected environmentally relevant chemical and physical properties of DNAN, NTO, TNT, and RDX, including solubility at $25^{\circ} \mathrm{C}$, octanol-water partition coefficient (Kow), and soil organic carbon adsorption coefficient $\left(K_{o c}\right)$.

\begin{tabular}{|l|c|c|c|c|}
\hline \multicolumn{1}{|c|}{ Property } & DNAN & NTO & TNT & RDX \\
\hline Solubility, mg L-1 & $276.2^{\mathrm{a} 1}$ & $16642.0^{\mathrm{c} 2}$ & $100.5^{\mathrm{a} 3}$ & $59.9^{\mathrm{a}} 3$ \\
\hline Log Kow & $1.7-1.92^{\mathrm{b}}, 1.64^{\mathrm{a}} 4$ & $0.37-1.03^{\mathrm{b} 4}$ & $1.6-1.84^{5}$ & $0.81-0.87^{5}$ \\
\hline Log $K_{o c}$ & $3.11^{\mathrm{a}} 6,2.2^{\mathrm{b} 7}$ & $3.03^{\mathrm{a}} 6,2.1^{\mathrm{b} 7}$ & $3.2^{3}$ & $0.88-2.4^{3}$ \\
\hline
\end{tabular}

a measured; b estimated; $\mathrm{c}$ interpolated from measured values

${ }^{1}$ (Boddu et al. 2008); ${ }^{2}$ (Spear et al. 1989); ${ }^{3}$ (Brannon and Pennington 2002); ${ }^{4}$ (Sokkalingam et al. 2008); 5 (Yoon et al.. 2005); 6 (R. Pesce-Rodriguez, unpublished); ${ }^{7}$ (Chakka et al. 2010).

Little is known about the phototransformation of DNAN and NTO either as solids or in solution. This process is important because sunlight might chemically alter the surface of the IM compound to produce other compounds with different solubilities or toxicities. Taylor et al. (2010) discovered this to be the case for TNT and RDX. Preliminary phototransformation studies of DNAN and NTO solutions made using an accelerated program in an Atlas Ci5000 system (uses a xenon lamp and a 6-month cycle equivalent to 2 years of real-time exposure in southern Florida) indicated a half-life of less than 3 days for DNAN and NTO (R. Pesce-Rodriguez, unpublished data). These data suggest that in the presence of light, DNAN and NTO in solution are more stable than TNT but are less stable than RDX. Rao et al. (2013) observed even faster phototransformation for DNAN, with half-lives ranging between 0.11 and 1.51 days. Hydrolysis studies found that without sunlight, DNAN and NTO are stable in neutral, acidic, and basic solutions (R. Pesce-Rodriguez, unpublished data). Observed products of phototransformation, according to Le Champion et al. (1999a), include nitrites, nitrates, and carbon dioxide. 
Figure 1. Plots of the solubility of (a) DNAN and (b) NTO as a function of temperature. Data are from studies conducted at the U.S. Army Construction Engineering Research Laboratory

(CERL) (Boddu et al. 2008), the Army Research Laboratory (ARL) (R. Pesce-Rodriguez, unpublished), the Defense Science and Technology Organization (Smith and Cliff 1999), and others.
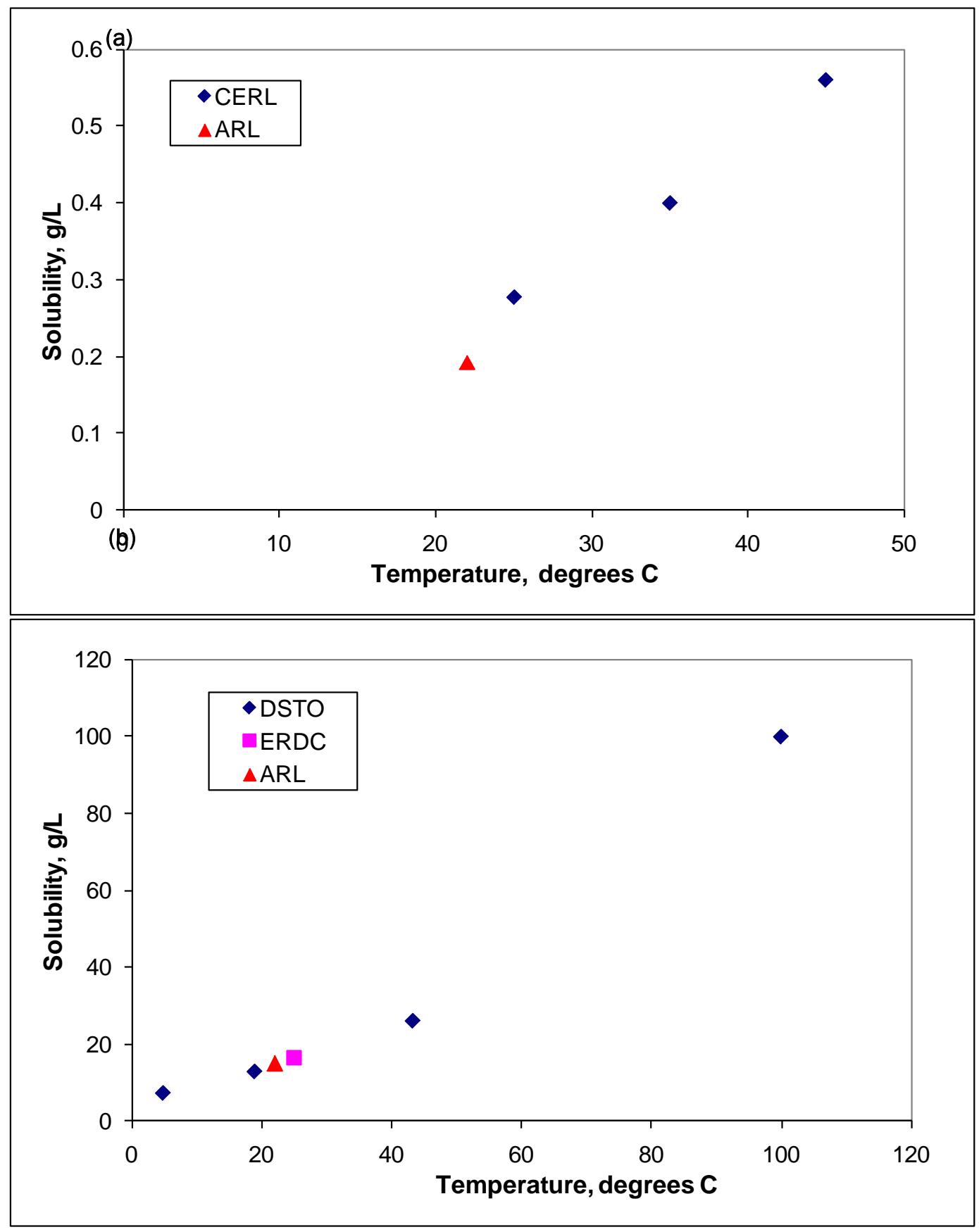

Outdoor dissolution tests of Comp B and TNT showed that phototransformation of the explosive pieces was a major loss pathway (Taylor et al. 2010). One-half to two-thirds of the mass dissolved was unaccounted for, and losses scaled closely with surface area (Figure 2). A 
similar phototransformation process may be occurring on the IM pieces we placed outside to dissolve.

For TNT and Comp B, the dissolved colored surface products were associated with large pre-solvent peaks. Analyses of the water samples showed the presence of 2-amino-4,6-dinitrobenzoic acid at concentrations similar to or larger than the TNT concentrations. As 2-amino-4,6-dinitrobenzoic acid is a known transformation product of TNT, this compound could account for the missing TNT mass. Because 2-amino-4,6-dinitrobenzoic acid is very polar, it is not quantified by Method 8300B (U.S. EPA 2006) and elutes in the pre-solvent peak area of the chromatogram (Taylor et al. 2010).

We proposed identifying and quantifying any phototransformation products occurring on IM chunks, but this portion of our project was not funded. If monies are available, we think that it is important to do this work as these phototransformation products are soluble and mobile; and we do not know what they are.

Figure 2. Mass loss measured by high precision liquid chromatography (HPLC) versus measured mass loss by electronic balance for TNT and Tritonal (a TNT-aluminum explosive) samples (Taylor et al. 2010).

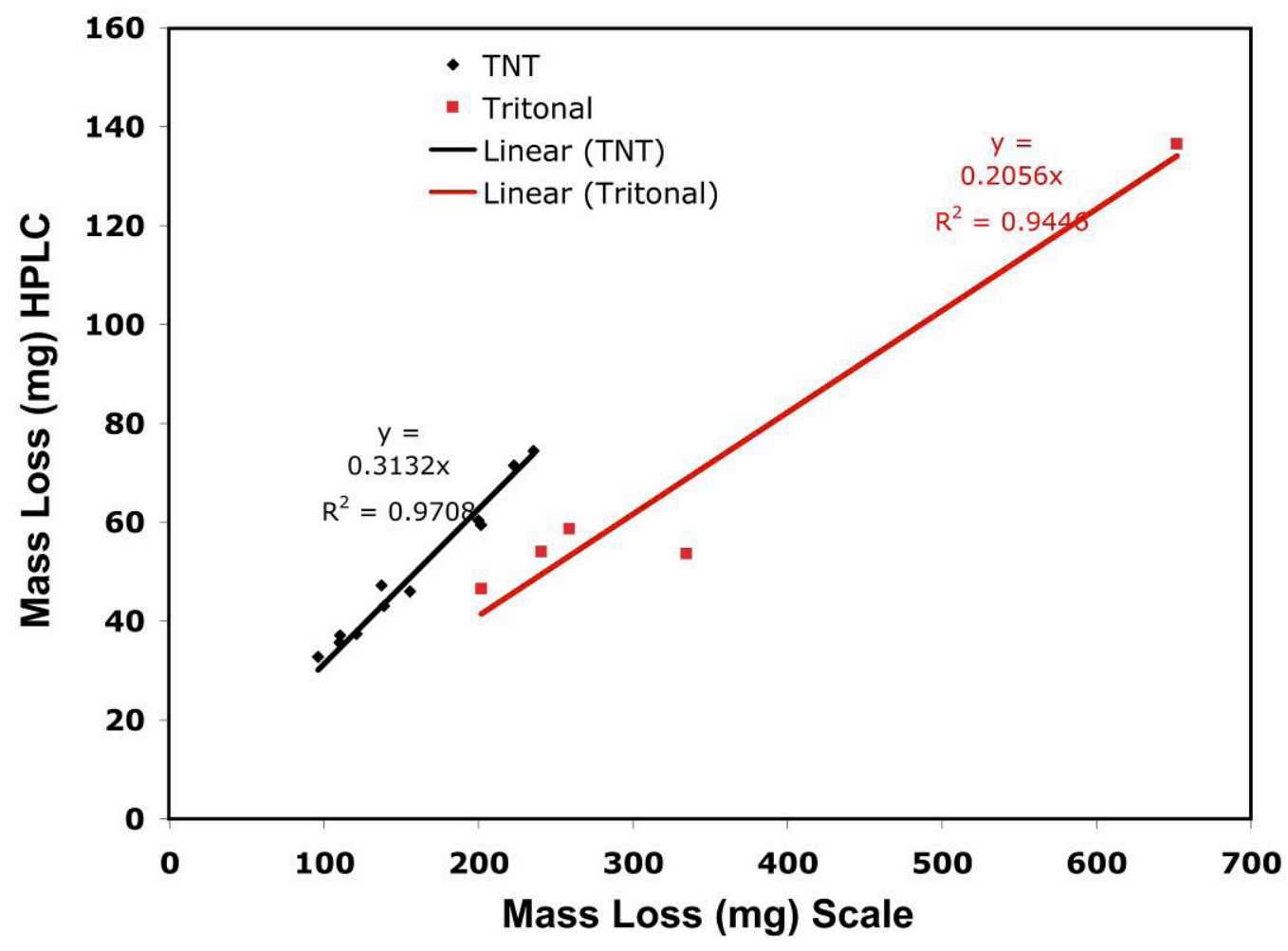


Once in solution, DNAN, NTO, and their transformation products will experience reactive transport through the soil. En route to ground water, they can undergo irreversible and reversible adsorption by different mineral and organic phases in the soil, transformation, volatilization, and biouptake. The importance of each of these processes for the fate of a compound can be evaluated using the soil adsorption coefficient $\left(K_{d}\right), K_{O C}, K_{o w}$, Henry's Law constant, the one-electron standard reduction potential, and the first-order transformation rate constant (k), among others. Table 1 provides values for some of these parameters for DNAN and NTO and for common explosives TNT and RDX.

The affinity of the energetics to be sorbed onto soil surfaces is characterized by the soil adsorption, or partitioning, coefficient, $\mathrm{K}_{\mathrm{d}}$. In particular, organic matter (OM), phyllosilicate clays, and iron and aluminum oxides and hydroxides adsorb organic compounds due to their high surface areas (Dontsova et al. 2009a). As values of $K_{d}$ increase, the chemical resides mostly on soil surfaces and little is transported downward in the moving pore water. As most of the adsorption of organic contaminants in soils can be attributed to soil organic carbon, $K_{d}$ values are often normalized to soil organic carbon content, and the resulting parameter is called the soil organic carbon adsorption coefficient $\left(\mathrm{K}_{\mathrm{OC}}\right)$. This parameter is estimated for the compound and then used to calculate adsorption to other soils, depending on their carbon content. There is little information about soil adsorption coefficients for the IM compounds. Recent work reports $K_{d}$ of $2.7 \mathrm{~cm}^{3} \mathrm{~g}^{-1}$ for DNAN and less than $0.1 \mathrm{~cm}^{3} \mathrm{~g}^{-1}$ for NTO in soil $(2.08 \%$ organic carbon [OC]), indicating that natural attenuation may be an important mechanism for DNAN remediation (Hawari et al. 2011, 2012). However, more work is needed to measure how chemical and biological transformation would affect the measured KoC values (as in the standard ASTM E1195-01 method [ASTM International 2008], adsorption is determined by the difference between initial and equilibrium concentrations of a compound in solution) and to determine the mechanisms involved. For example, RDX tends to partition to OC (Tucker et al. 2002; Dontsova et al. 2009a) while TNT exhibits very complex sorption behaviors, which include adsorption in clay interlayer surfaces (Haderlein et al. 1996) and irreversible adsorption to OM following transformation (Thorn and Kennedy 2002).

Unlike most other explosives, which are polar but non-ionic compounds, NTO is an acid with an acid dissociation constant ( $\mathrm{pK}_{\mathrm{a}}$ ) of 3.7-3.76 
(Chipen et al. 1966; Le Campion et al. 1997) and will be negatively charged at environmentally relevant pHs (Smith and Cliff 1999). As both organic and mineral soil surfaces tend to have a net negative charge, we would predict low affinity of NTO for soils and greater mobility in the environment. However, negatively charged organics can be adsorbed in soils through cation bridging.

The octanol- water partition coefficient is a measure of the tendency of the chemical to bio-accumulate (Boddu et al. 2008) and an indicator of its affinity for soil OM. Relatively low reported $\mathrm{K}_{\text {ow }}$ values (Table 1) indicate that these compounds would not be strongly adsorbed by OM in soils through non-polar interactions. However, they can still experience specific sorption, as has been shown for TNT and the DNTs (2,4- and 2,6dinitrotoluene) (Thorn et al. 2001; Thorn et al. 2008). Based on the $\mathrm{K}_{\text {ow }}$ values, one would expect NTO to be more mobile than DNAN and to exhibit similar environmental behaviors to RDX.

Relatively low volatility, as measured by Henry's constants (logH of -3.25 to -4.40 for DNAN and - 11.38 for NTO) (Sokkalingam et al. 2008), indicates that transport in a gas phase will not be important for the total mass flux of IM compounds in soils. One-electron standard reduction potential $\left(E_{m}\right)$ is a measure of how easily nitro groups in these compounds reduce to amino groups (Uchimiya et al. 2010), a common first step in transformation of many explosive compounds. Boparai et al. (2010) observed faster reduction for explosives having more positive one-electron reduction potentials. Measured electron standard reduction potential for DNAN (-0.40 volt) was similar to 2,4-DNT, which is readily reduced in soil environments. Note that transformation products of the compound can have different environmental and toxicity properties than the original contaminant.

Microbial degradation studies of NTO in soils show nitroreduction followed by the ring cleavage of the primary amine, 5-amino-1,2,4-triazol-3one (ATO) (Le Campion et al. 1999b). At optimal conditions, the nitroreduction of NTO was complete in $24 \mathrm{hr}$, while the degradation of ATO required 2 weeks of incubation. The end products of the biodegradation were carbon dioxide (40\%), urea, and a polar compound assumed to be hydroxyurea. 
In summary, there is insufficient information about soil adsorption for the IM compounds. Both the extent of sorption and the mechanisms involved are not yet known. $\mathrm{K}_{\mathrm{ow}}$ has been estimated and measured (Sokkalingam et al. 2008), but the Koc values that are currently available were computationally derived (Chakka et al. 2010) from $K_{\text {ow, }}$ which can result in unreliable values, particularly for polar compounds; and soil adsorption was measured for only one soil (Hawari et al. 2011, 2012), making it difficult to generalize how IM compounds will behave in other soils. To address these knowledge gaps, we will measure DNAN and NTO adsorption coefficients $\left(\mathrm{K}_{\mathrm{d}}\right)$ for multiple soils having a range of properties and will normalize the values to their organic carbon content to determine $\mathrm{Koc}_{\mathrm{o}}$. If these parameters are known, the fate of the chemical in the soil system can be modeled. Because soils are an important mechanism for natural remediation of organic and inorganic contaminants, values for $\mathrm{K}_{\mathrm{d}}, \mathrm{k}$, and $\mathrm{K}_{\mathrm{OC}}$ are critical for understanding the fate and transport of the IM compounds.

\subsection{Objectives and approach}

We seek to measure how quickly DNAN and NTO dissolve in IM formulations and how solutions of these IM explosives interact with different types of soils. Because both dissolution and solution- soil interactions are determined by a suite of parameters, we are using a multifaceted approach to study of these processes. Given a mass of IM compounds scattered on the range, our work will help determine the dissolved IM masses, their subsequent transport and fate, and their likelihood of reaching groundwater.

To measure the dissolution, phototransformation, and soil adsorption properties of DNAN, NTO, and IM formulations that contain them (IMX101, IMX104, and PAX21), we

1. used light and electron microscopy to characterize particles of DNAN, NTO, and detonation residues of IM formulations that contain them;

2. measured the dissolution of detonation residues of IM formulations in a controlled laboratory setting and in the field where they were exposed to rainfall and sunlight;

3. measured the phototransformation of DNAN and NTO in solution and from solid IM formulations set outside to degrade and dissolve;

4. determined adsorption behavior of IM compounds in a range of soils; and

5. measured the transport behavior of DNAN and NTO in representative soils, which helped us correlate their transport and fate behavior to soil properties. 


\section{Dissolution Studies}

\subsection{Materials and methods}

\subsubsection{IM particle collection}

All particles came from partially detonated rounds and were collected in the field. We obtained detonation residues of IMX101 from tests conducted on $105 \mathrm{~mm}$ howitzer rounds by National Technical Systems and then collected PAX21 and IMX104 residues after low-order detonations of 60 and $81 \mathrm{~mm}$ mortars, respectively (Walsh et al. 2013, 2014).

Table 2. IM formulations containing DNAN and NTO in advanced stages of development or ready for fielding.

\begin{tabular}{|l|l|l|l|}
\hline \multicolumn{1}{|c|}{ IM } & Replaces & \multicolumn{1}{c|}{ Components } & \multicolumn{1}{c|}{ Used In } \\
\hline IMX101 & TNT & $\begin{array}{l}43.5 \% \text { DNAN, 36.8\% NQ, } \\
19.7 \% \text { NTO }\end{array}$ & $\begin{array}{l}\text { Qualified by the U.S. Army as the main fill } \\
\text { in the 155 mm M795 Artillery Projectile }\end{array}$ \\
\hline IMX104 & Comp B & $\begin{array}{l}53 \% \text { NTO, 31.7\% DNAN, } \\
15.3 \% \text { RDX }\end{array}$ & $\begin{array}{l}\text { Used by the U.S. Army for 60, 81, and 120 } \\
\text { mm mortars and sub-munitions }\end{array}$ \\
\hline PAX21 & Comp B & $\begin{array}{l}34 \% \text { DNAN, 30\% AP, } \\
30.75 \% \text { RDX }\end{array}$ & $\begin{array}{l}\text { Qualified and fielded in the U.S. Army 60 } \\
\text { mm mortar system }\end{array}$ \\
\hline
\end{tabular}

Some data from Fung et al. (2010), Wilson (2007), Pelletier et al. (2010), and Coppola (2007).

\subsubsection{Imaging}

We used a SkyScan $1172 \mathrm{X}$-ray microtomograph to image the IM formulations. We ran the instrument at a $40 \mathrm{kV}$ by using a $250 \mu \mathrm{A}$ source current. A 1.3 megapixel X-ray camera took a sequence of shadow transmission images (radiographs) of each IM particle as it rotated on the stage. From these radiographs, cross sectional images perpendicular to the z-axis of the particle were reconstructed using a modified Feldkamp cone-beam algorithm, creating a complete three-dimensional (3-D) representation of the internal microstructure of the particle. We quantified the areas of the matrix and of the crystal components in each section using Image J (Rasband 2012). We calculated the volumes by multiplying these area measurements by the spacing between cross sections ( 4 to $7 \mu \mathrm{m}$ ). This technique required no sample preparation.

\subsubsection{Indoor drip tests}

We used well-controlled laboratory drip experiments to obtain dissolution data on particles of detonated IMX101, IMX104, and PAX21. Our experi- 
mental design mimicked field conditions on training ranges, where rainfall dissolves spatially isolated particles and chunks of explosives scattered on the soil surface (Taylor et al. 2009a). We placed a massed piece of the explosive on a glass frit, dripped water on the particle at a given rate, and analyzed the effluent using HPLC (Figure 3a). No soil was involved in the tests, allowing us to determine the dissolution as a function of the particle mass and the water volume interacting with the particle. Figure 3 a shows the syringe pump used in these tests.

Figure 3. Photos of (a) the laboratory drip tests and of (b) the outdoor setup.
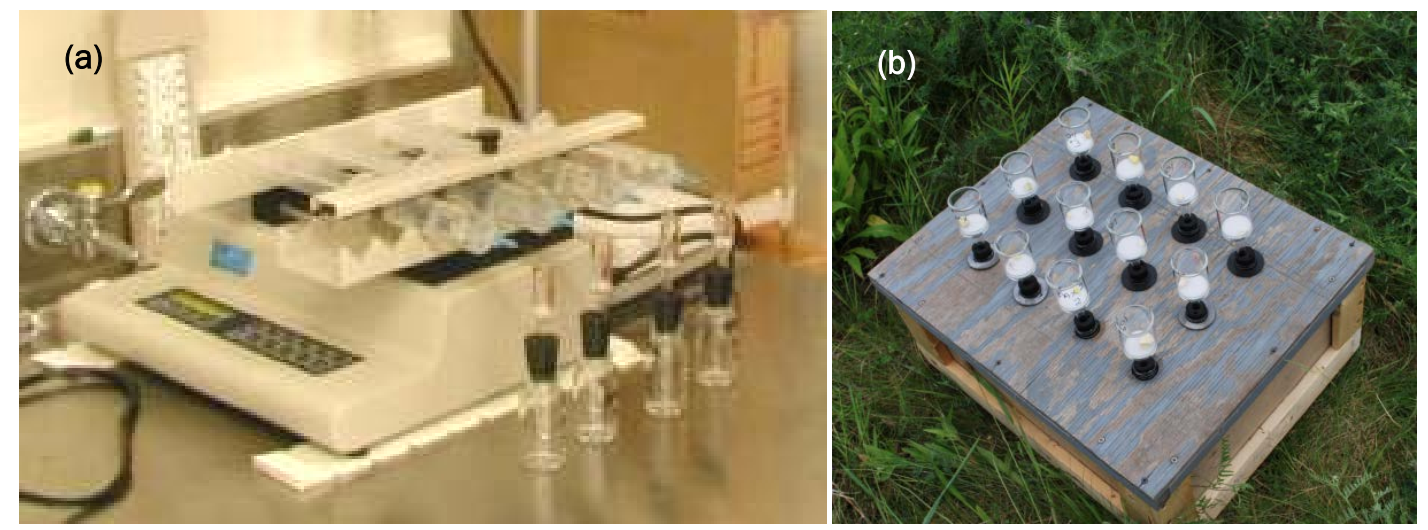

\subsubsection{Outdoor drip tests}

We also conducted dissolution tests using centimeter-size chunks of high explosives that we placed outside to weather under natural conditions at the Cold Regions Research and Engineering Laboratory (CRREL) facility in Hanover, NH (Figure 3b). These tests closely simulated the dissolution of isolated high explosive pieces on range soils.

In J une 2013, we placed 5 chunks of IMX101, 5 chunks of IMX104 and 2 chunks of PAX21 outside in $4 \mathrm{~cm}$ diameter Buchner funnels (Figure $3 \mathrm{~b}$ ). The funnels were attached to $1 \mathrm{~L}$ bottles with a \#4 rubber stopper that was fitted with two holes-one for the funnel stem and the other for air exchange. The bottles fitted snuggly into an insulated wooden box. The box kept the bottles from tipping over or being damaged and also helped to keep them cool in summer and warm in winter.

Rainwater or snowmelt that interacted with the high explosives was collected in the bottles. Periodically, we exchanged the bottles for clean ones and measured the volume and high-explosive concentration in the water. During the winter months, we placed the bottles in plastic bags to save the 
sample even if the bottle should break from ice formation. During each collection, we photographed the pieces of high explosives in situ to document changes in their appearance and size. This setup allowed the explosives to be exposed to conditions similar to those experienced by explosives on a range - where rain, snow, sun, and freeze- thaw cycles weather them -while allowing us to collect and analyze the dissolved high explosives and to monitor changes in their appearance. We set up our experiment in an enclosed, locked site next to a dedicated rain gauge and near the CRREL weather station, which records rainfall totals, temperature, and solar irradiance. We downloaded the rain data monthly.

\subsubsection{Analytical methods}

We analyzed by HPLC all solutions from the drip and the outdoor tests and, using an ion selective probe, analyzed solutions from PAX21 particles for perchlorate. We used two HPLC methods: Method 8330 (U.S. EPA 2006) to quantify DNAN, RDX, and HMX (a manufacturing impurity of RDX) and a second, described below, to quantify NTO and NQ. For both methods, we ran a 1 or 10 ppm standard every ten samples and ran blanks before and after each standard run to prevent carryover.

We used the U.S. Environmental Protection Agency's SW-846 Method 8330 (U.S. EPA 2006) to quantify DNAN, RDX, HMX, their cocontaminants, and breakdown products. We added DNAN to the commercially available 8330 standard (Restek) and used this solution for calibration. We used a Waters NovaPak C8 column $(3.9 \times 150 \mathrm{~mm})$ eluted at 1.4 $\mathrm{mL} \mathrm{min}^{-1}\left(28^{\circ} \mathrm{C}\right)$ with $85: 15$ water:isopropanol mix and detected by UV at $295 \mathrm{~nm}$. We added $1 \mathrm{~mL}$ of the effluent to $2 \mathrm{~mL}$ of deionized water and to 1 $\mathrm{mL}$ of acetonitrile and then filtered it through a $0.45 \mu \mathrm{m}$ Millipore syringe filter.

We prepared the samples for NTO and NQ by adding $1 \mathrm{~mL}$ of the effluent to $3 \mathrm{~mL}$ of acetonitrile containing $0.1 \%$ trifluoroacetic acid (TFA) and then filtered it through a $0.45 \mu \mathrm{m}$ Millipore syringe filter. The standard contained $1 \mathrm{~mL}$ of a $1 \mathrm{mg} \mathrm{L}^{-1}$ solution of NTO in acetonitrile (AcN), $1 \mathrm{~mL}$ of a 1 $\mathrm{mg} \mathrm{L}^{-1}$ solution of NQ dissolved in water, and $2 \mathrm{~mL}$ of AcN containing $0.1 \%$ TFA. We used a Thermo Scientific Hypercarb column $(4.6 \times 150 \mathrm{~mm})$ eluted at $1.4 \mathrm{~mL} \mathrm{~min}^{-1}\left(28^{\circ} \mathrm{C}\right)$ with $95: 5$ methanol:water mix and detected by UV at $300 \mathrm{~nm}$. 
We quantified the AP using a Cole Parmer ion-selective electrode. To generate a standard line from which we could determine the AP concentration of the samples, we prepared and analyzed a series of concentrations from a perchlorate standard. Using a Mettler Toledo SevenEasy meter and a pH probe, we measured the $\mathrm{pH}$ of the water samples; and we completed a three-point calibration by using $\mathrm{pH} 4,7$, and 10 solutions.

\subsection{Results}

\subsubsection{Characterized particles of DNAN, NTO, and detonation residues of IM formulations}

Figure 4 shows optical images of DNAN, NTO, and the IM formulations and the compositions of the formulations. DNAN is a cream-colored particle composed of many small crystals. It abrades easily as evidenced by the powdered DNAN on its surface. NTO is a translucent crystal that occurs as about $500 \mu \mathrm{m}$, equidimensional grains, most of which look twinned and are highly reflective in visible light. IMX101 and 104 are white crystalline substances with visible NTO crystals embedded in the DNAN matrix. PAX21 has a yellow DNAN matrix caused by the addition of less than 1 wt.\% methyl nitroaniline with clearly visible 200-400 $\mu \mathrm{m}$ AP crystals.

IMX101, IMX104, and PAX21 are made by melt casting, a process where crystalline constituents (e.g., AP, NTO, NQ, and RDX) are added to a molten matrix (DNAN) and then cooled. Melt casting is also used to produce conventional high explosives, such as TNT and Comp B. In the case of Comp B, $100 \mu \mathrm{m}$ crystals of RDX are added to molten TNT. For IMX101, $360 \mu \mathrm{m}$ NTO and $300 \mu \mathrm{m}$ NQ crystals are added to molten DNAN; for IMX104, approximately $300 \mu \mathrm{m}$ NTO crystals and $4 \mu \mathrm{m}$ RDX crystals are added to DNAN; and for PAX21, 200- $400 \mu \mathrm{m}$ AP crystals and RDX crystals of two different sizes are mixed into DNAN (Pelletier et al. 2010; Rutkowski et al. 2010). 
Figure 4. Optical images of DNAN, NTO, IMX101, IMX104, and PAX21 and the compositions of the three IM formulations we studied.

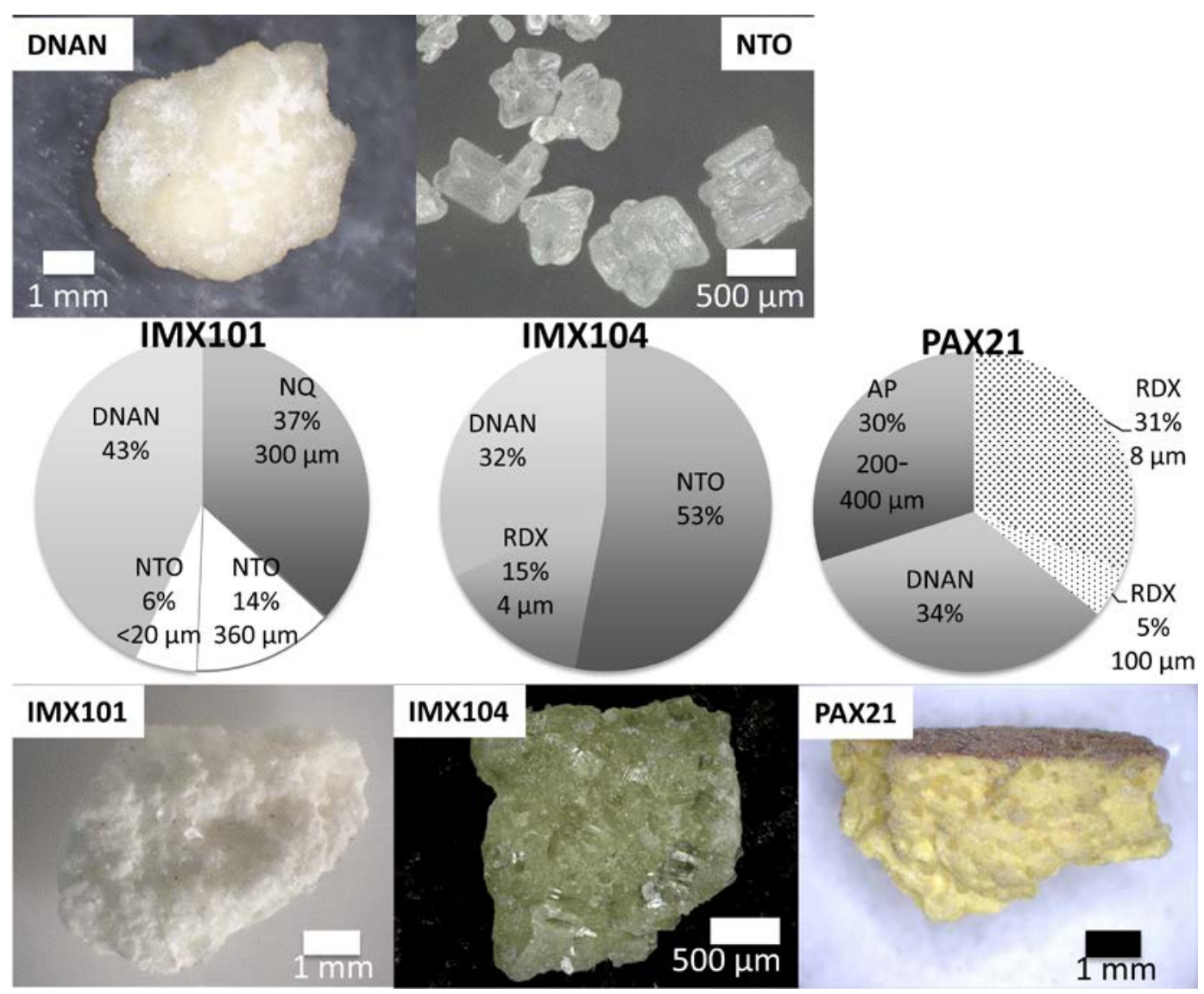

The IM formulations differ from Comp B in several important ways. First, most of their crystal constituents are much more soluble than their DNAN matrices, which is the opposite of Comp B where the RDX is less soluble than the TNT matrix. We surmised (correctly) that as NTO, NQ, and AP dissolve, they would leave holes in the DNAN matrix, thereby increasing DNAN's surface area and its dissolution rate (Figure 5). Second, because the solubility of NTO, NQ, AP and DNAN differ from each other by orders of magnitude, we expected these compounds to dissolve at different rates based on their solubility and on the fraction of their surface exposed to water. The 3-D relationship of the constituents and their relative crystal sizes in IM formulations is, therefore, important for understanding and modeling the rate at which IM particles dissolve. Figure 6 illustrates an example of the effect differences in solubility can have on particle structure. We found that the AP in PAX21 particles quickly dissolved, leaving a DNANRDX matrix. As the DNAN dissolved, the small, approximately $10 \mu \mathrm{m} R D$ crystals remained. 
Figure 5. Images of (a) the Comp B surface and of (b) an IMX104 particle. These images show that as the TNT matrix dissolves from Comp B, the less soluble RDX crystals jut from the surface and eventually fall off the particle. For IMX104, on the other hand, the DNAN matrix is less soluble than the crystal constituents and dissolution produces hole-riddled particles.

\section{Comp B}

IMX104-3

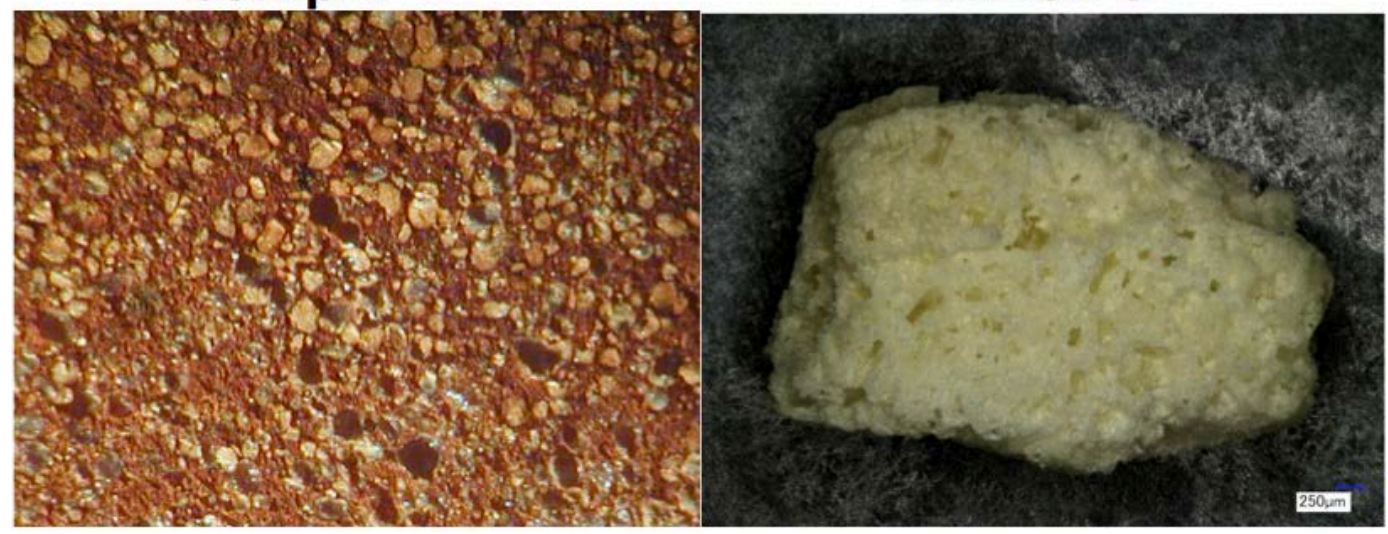

Figure 6. PAX21 particles: (a) detonation residue; (b) AP completely dissolved; (c) DNAN almost completely dissolved; and (d) RDX crystals remaining.

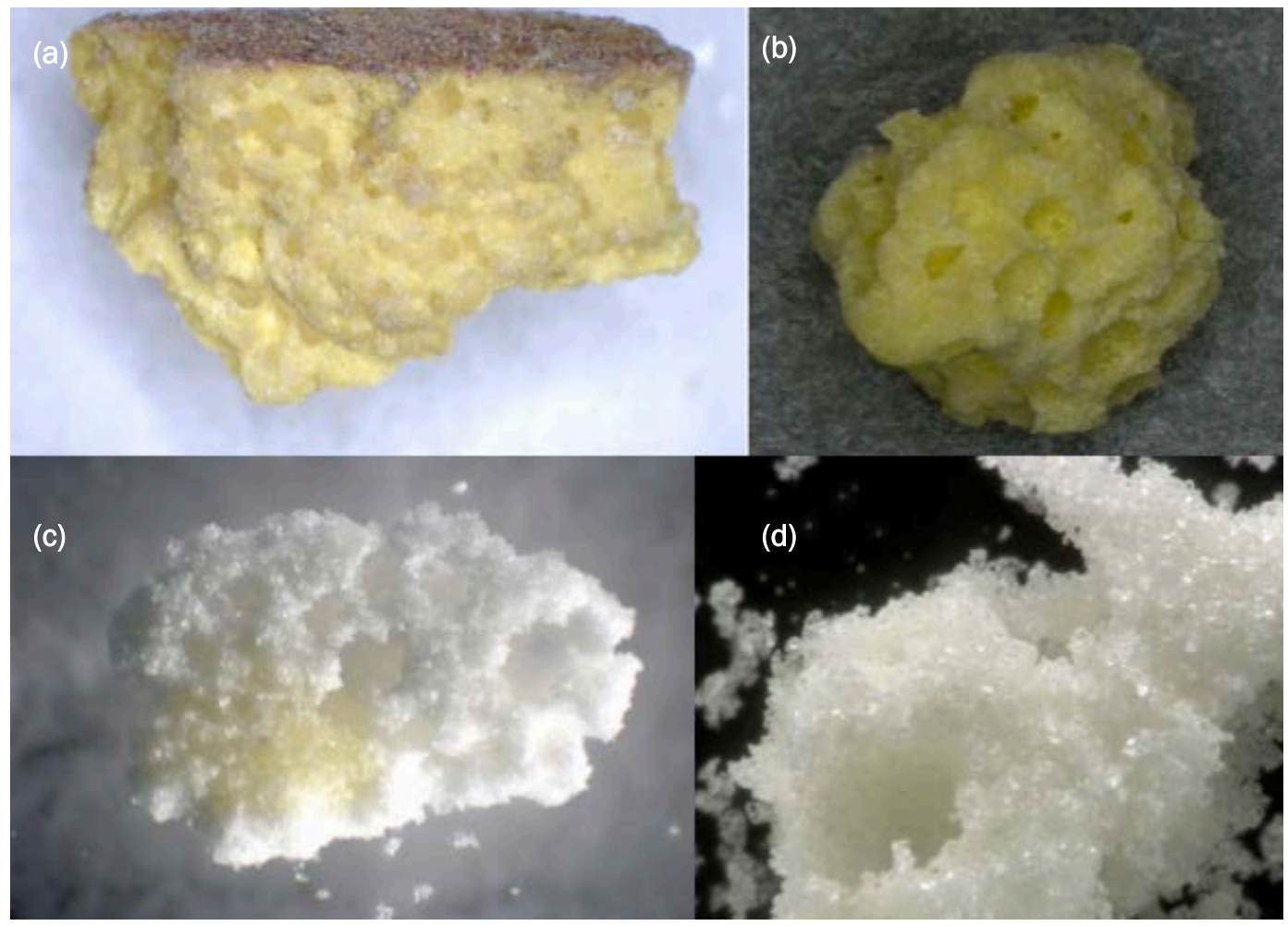

To image the 3-D distribution of the various components in IMX101, IMX104, and PAX21, we used micro-computed tomography ( $\mu \mathrm{CT}$ ). Figure 7 shows a radiograph (a, c, e, and $g$ ) and a reconstructed cross section (b, $\mathrm{d}$, f, and h) for IMX101, IMX104, and PAX21. The same PAX21 particle is shown twice, before and after a half hour submersion in water. The round- 
ed AP crystals are clearly visible in the PAX21 radiographs because AP has a higher X-ray attenuation that DNAN and RDX. This is not the case for IMX101 (Figure 7a) and IMX104 (Figure 7c) as the X-ray attenuations for DNAN, NTO, RDX, and NQ are all similar.

In contrast, the reconstructed cross sections of the IM formulation do show the constituent crystals. IMX101 has square shaped NQ crystals and larger, brighter NTO crystals in a darker DNAN matrix (Figure 7b). Cracks in the DNAN matrix are also visible. Reconstructed cross sections of IMX104 (Figure 7d) also show the NTO crystals and fractures in the particle.

Figure 7. Shown are radiographs and reconstructed cross sections of IMX101 ( $a$ and $b$ ), IMX104 (c and d), and PAX21 (e and f). Also shown is the same PAX21 particle after a halfhour submersion in water ( $g$ and $h$ ). The scale bar applies to only the cross sections. The diameter of the post used to mount the samples for the radiographs was $3 \mathrm{~mm}$.

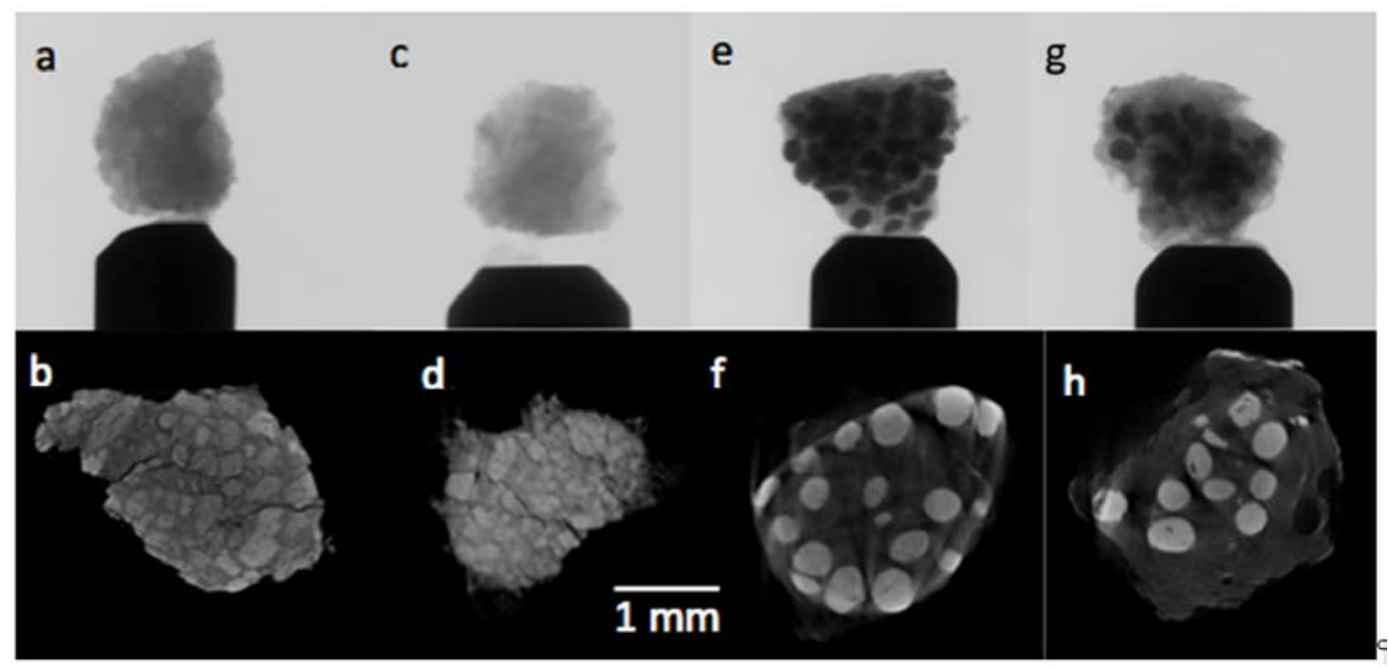

The AP crystals in reconstructed cross sections of PAX21 are white, large, rounded objects in a grey matrix (Figure 7f). The smaller bright phases (hard to see) are $100 \mu \mathrm{m}$ RDX crystals. This particle was placed in water for $30 \mathrm{~min}$ and then reimaged (Figure $7 \mathrm{~g}$ and $\mathrm{h}$ ). Using the computed cross sections taken pre- and post- submersion, we calculated the volume of the particle and of the AP crystals within it. Initially the PAX21 particle contained 110 AP crystals that occupied $5 \mathrm{~mm}^{3}$, or $24 \%$, of the $21 \mathrm{~mm}^{3}$ particle volume. After a half hour in water, $63 \mathrm{AP}$ crystals remained; and these occupied $2 \mathrm{~mm}^{3}$, or $12 \%$, of the post-submersion particle volume $\left(16 \mathrm{~mm}^{3}\right)$. A decrease in the percent $\mathrm{AP}$ volume after submersion confirms that the $\mathrm{AP}$ dissolved preferentially. The AP crystals within $0.5 \mathrm{~mm}$ from the periphery dissolved, and those crystals $0.6 \mathrm{~mm}$ from the edge partially dissolved. 
The sequence of cross sections showed that AP crystals are about $50 \mu \mathrm{m}$ apart and that only a few actually touch.

To determine if the fractures we saw in the $\mu \mathrm{CT}$ cross sections formed during detonation, we imaged and compared four manufactured and four detonated particles of IMX101, IMX104 and PAX21 (Figure 8). We saw internal cracks in the detonated particles and none in the $\mu$ CT cross sections of the manufactured pieces, indicating that detonation fractured the particles. The fractures were generally in the DNAN and followed the perimeters of the AP, NTO, or NQ crystals. A few of the fractures extended through the entire particle and could be followed in many successive cross sections.

Figure 8. Images of undetonated, detonated, and wetted IM particles. Note the fractures in the detonated particles and the loss of components in the wetted particles.

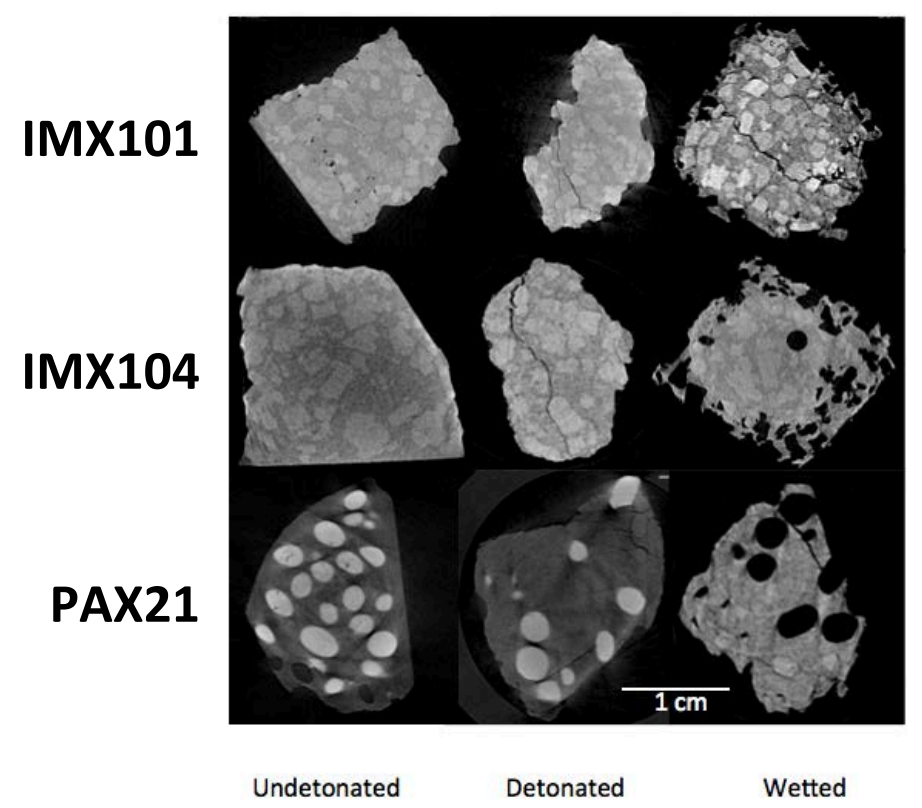

\subsubsection{Laboratory tests to measure the dissolution of IM detonation residues}

We conducted two drip dissolution tests, one that dripped a total of about $20 \mathrm{~mL}$ of water on samples that were imaged using $\mu \mathrm{CT}$ and the second that dissolved the particles completely. We weighed all IM samples at the beginning of the tests and massed the $\mu \mathrm{CT}$ samples at the end using a Mettler A230 balance $(1.0 \pm 0.1 \mathrm{mg})$. 
The first tests involved imaging individual IM particles, dripping water on them, reimaging them, and repeating this procedure numerous times. We placed each particle in a scored plastic pipette tip. The plastic tip did not interfere with the $\mu C T$ scans, it held the particle in place and the scoring allowed us to place the samples in the scanner in the same orientation each time. Although the pipette tip stabilized the particle, some of the particles did move as they dissolved. The water dripped on the particle flowed out the tip bottom to the sample vial.

For the second tests, we placed each particle on a glass frit within a Buchner funnel, dripped water on the particle at $0.5 \mathrm{~mL} \mathrm{hr}^{-1}$ using a syringe pump, and analyzed the effluent using HPLC. Four particles of IMX101 and IMX104 were run continuously until the particle dissolved (month time scales). These tests are similar to those run on high explosives (Lever et al. 2005; Taylor et al 2009b). We did not run similar tests on PAX21 because its use was discontinued when Walsh et al. (2013) found that AP was not consumed during high order detonations.

For both of these tests, we massed and analyzed the Milli-Q water interacting with the IM particles. One major difference between the two dissolution tests was that particles placed in the pipette tips were always struck by the drops whereas those in the glass frit were only struck some of the time. No soil was present in either type of test.

\subsubsection{Dissolution of IM compounds tracked by $\mu C T$.}

Figure 9 shows the cumulative mass dissolved in the water dripped on the $\mathrm{CCT}$-imaged IMX101, IMX104, and PAX21 particles. For IMX101, more NTO and NQ dissolved than DNAN; and more NTO dissolved from each particle than NQ despite there being more NQ in the particle. For IMX104, the dissolved masses of the three components are different enough to be well separated on the plot with NTO > DNAN > RDX. For PAX21, AP dissolved quickly and completely, followed by DNAN and RDX. The compounds dissolved in accordance with their solubility. 
Figure 9. Cumulative mass dissolved in the water dripped on the $\mu$ CT-imaged (a) IMX101, (b) IMX104, and (c) PAX21 particles.

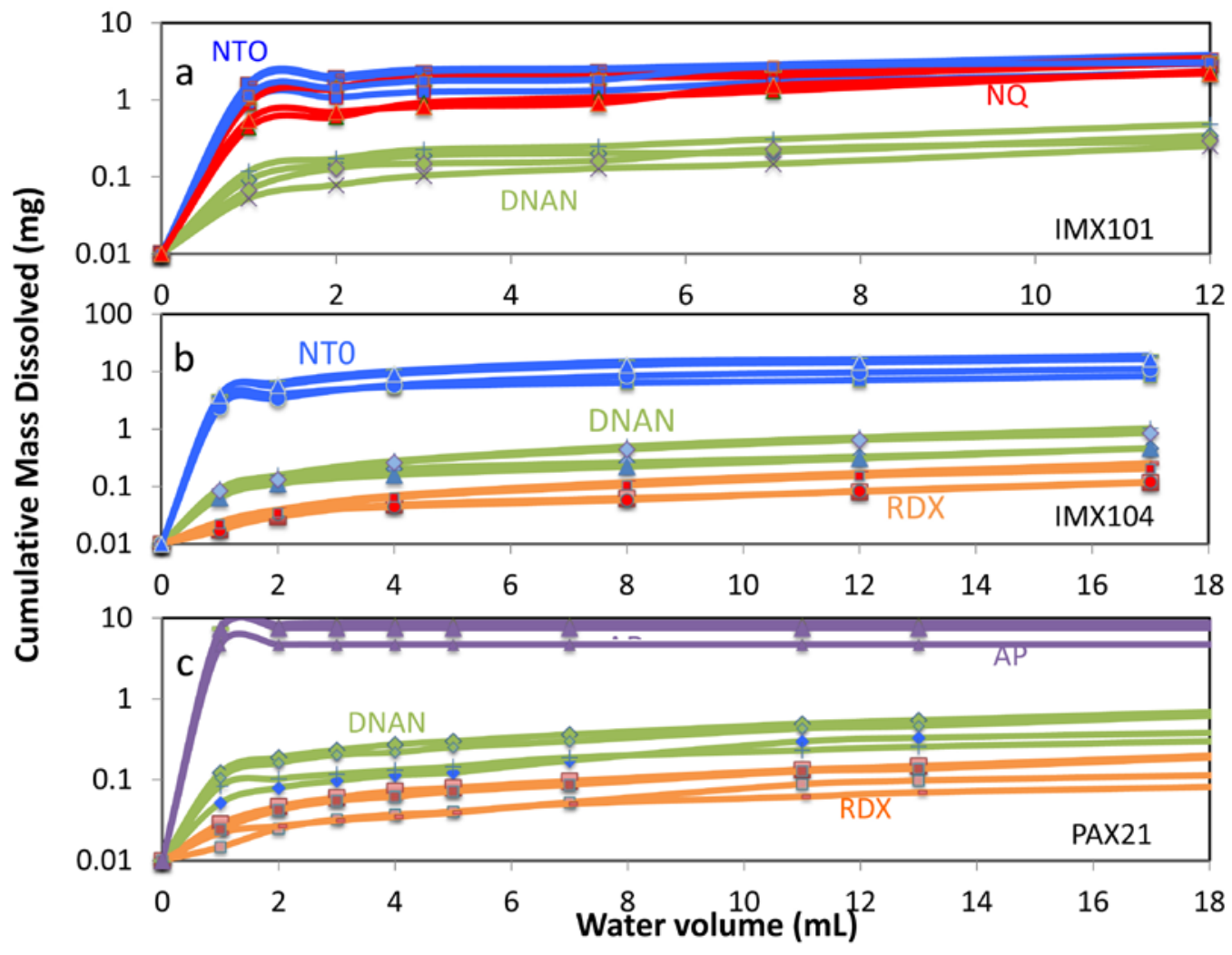

The $\mu \mathrm{CT}$ reconstructed cross sections show the physical changes undergone during dissolution of each IM formulation. Figure 10 shows a cross section of each formulation and how these changed as a function of water interaction. For IMX101, as the NTO and NQ crystals dissolved, they left thin bridges of DNAN matrix that can break and dissolve. For IMX104, the dissolution of NTO is more noticeable as NTO composes over $50 \%$ of the formulation. Here again, the DNAN matrix maintains the outline of the former crystals. Note that both IMX101 and 104 have well defined cracks and yet dissolution appears to proceed from the outside inward and not preferentially along the fractures. For PAX21, the bright AP crystals are dissolved after contact with only a small volume of water $(2 \mathrm{~mL})$. Although fractures are present in PAX21, these not as well developed as in the other two formulations; and it is not clear how the water accessed the AP. After the AP dissolved, the RDX crystals could be seen. They are the small grains that are slightly brighter than the DNAN matrix. Movies made from the $\mu \mathrm{CT}$ sections are available in the supplementary materials of Taylor et al. (2013, 2015). 
Figure 10. Change in the cross section of each formulation with increased water interaction.

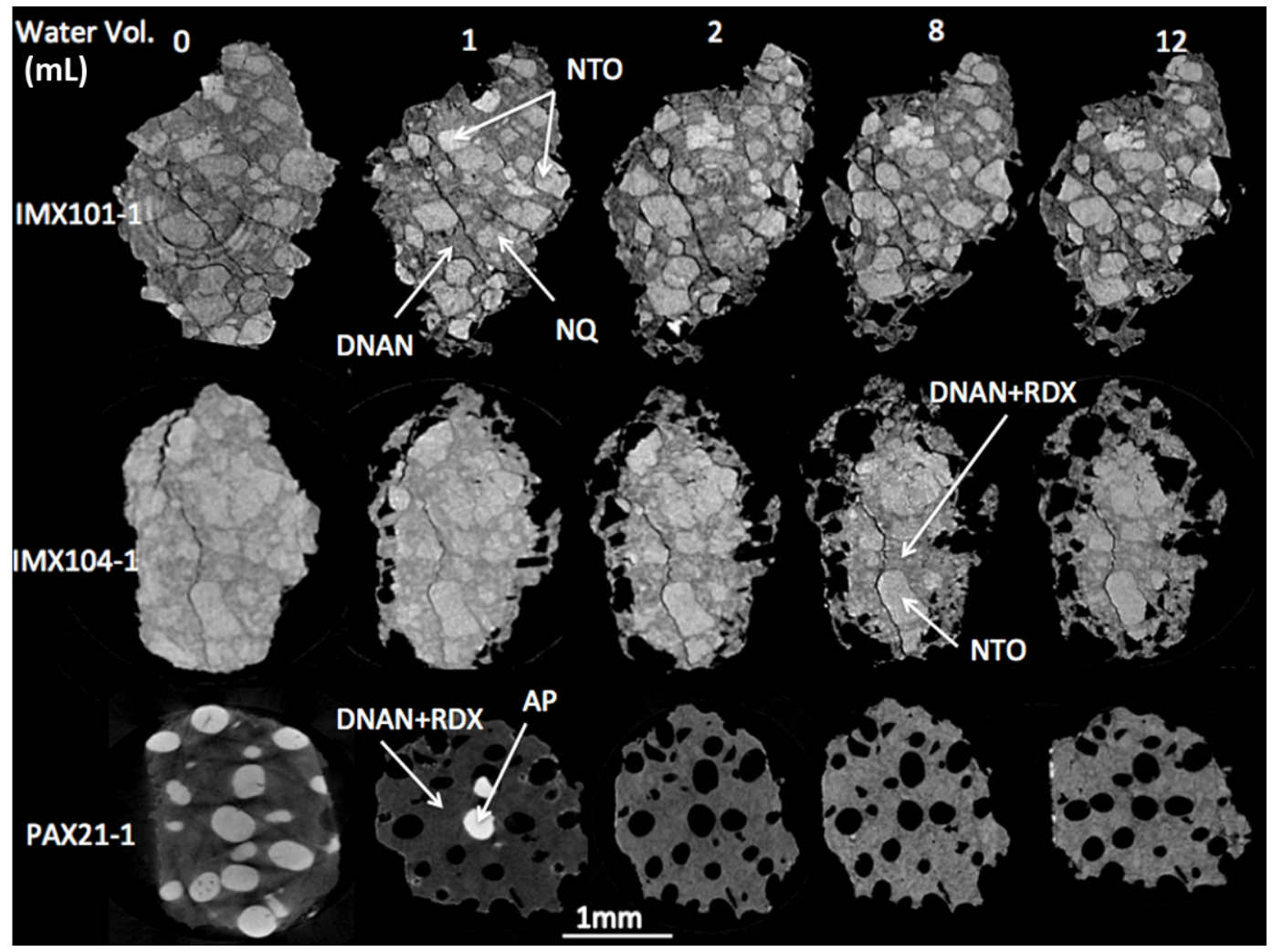

From the $\mu \mathrm{CT}$ cross-section images, we calculated the area of the particle and of the crystals in each section. We then estimated the volume of the whole particle and of its crystal components by multiplying each crosssectional area by the spacing between sections (Table 3).

Figure 11a plots changes in particle and crystal volumes versus water volume for the four IMX101 particles. Note the large change in crystal volume caused by the first $\mathrm{mL}$ of water. Over the course of the test, the ratio of matrix to crystal changed from 2 to 3 , in keeping with the fact that NTO and NQ are more soluble than DNAN.

We estimated the density of the formulations by plotting the initial volume versus mass of each particle and fitting a line to the data (Figure 11b). This procedure yielded initial densities of $1.69 \mathrm{~g} \mathrm{~cm}^{-3}$ for IMX101, $1.67 \mathrm{~g} \mathrm{~cm}^{-3}$ for IMX104, and $1.56 \mathrm{~g} \mathrm{~cm}^{-3}$ for PAX21. Because the density of the formulation should change during dissolution if dense or less dense phases preferentially dissolve, we plotted the final masses and volumes and obtained densities of $1.55 \mathrm{~g} \mathrm{~cm}^{-3}$ for IMX101, $1.67 \mathrm{~g} \mathrm{~cm}^{-3}$ for IMX104, and $1.32 \mathrm{~g} \mathrm{~cm}^{-}$ 3 for PAX21. 
Finally, to check for mass closure, we compared the dissolved masses measured by HPLC with the difference between the initial and ending masses measured by analytical balance (Table 3). For IMX101 and 104, we had good mass balance with relative errors generally less than $5 \%$. The errors were larger for PAX21 but below $10 \%$.

Table 3. Mass balance for IMX101, IMX104, and PAX21 particles used in the $\mu \mathrm{CT}$ drip dissolution tests. Also tabulated are the calculated volumes for each particle.*

\begin{tabular}{|c|c|c|c|c|c|c|c|c|}
\hline & \multicolumn{3}{|c|}{ Mass (mg) Scale } & \multirow{2}{*}{$\begin{array}{c}\text { Mass (mg) } \\
\text { HPLC }\end{array}$} & \multirow{2}{*}{$\begin{array}{c}\text { Relative Error } \\
(\%)\end{array}$} & \multicolumn{3}{|c|}{ Volume $\left(\mathrm{mm}^{3}\right)$} \\
\hline & $t=0$ & $t=$ End & Difference & & & $t=0$ & $t=$ End & Difference \\
\hline \multicolumn{9}{|l|}{ IMX101 } \\
\hline$\# 1$ & 18.97 & 12.51 & 6.46 & 6.30 & 2.48 & 12.24 & 8.23 & 4.0 \\
\hline$\# 2$ & 18.65 & 13.71 & 4.94 & 4.89 & 1.01 & 11.53 & 8.58 & 2.9 \\
\hline \#3 & 24.03 & 15.88 & 8.15 & 7.82 & 4.05 & 14.10 & 10.09 & 4.0 \\
\hline \#4 & 20.37 & 14.50 & 5.87 & 5.54 & 5.62 & 12.50 & 9.61 & 2.9 \\
\hline A & 35.11 & & & & & 19.91 & & \\
\hline$B$ & 16.58 & & & & & 9.48 & & \\
\hline C & 3.57 & & & & & 1.97 & & \\
\hline D & 36.81 & & & & & 22.50 & & \\
\hline$\rho\left(\mathrm{mg} / \mathrm{mm}^{3}\right)$ & & & & & & 1.67 & 1.55 & \\
\hline \multicolumn{9}{|l|}{ IMX104 } \\
\hline \#1 & 18.18 & 8.86 & 9.32 & 8.93 & 4.17 & 10.99 & 5.47 & 5.5 \\
\hline \#2 & 22.37 & 10.53 & 11.84 & 11.69 & 1.30 & 13.33 & 6.03 & 7.3 \\
\hline \#3 & 52.57 & 33.3 & 19.27 & 20.03 & 3.95 & 31.08 & 19.52 & 11.6 \\
\hline \#4 & 37.32 & 19.81 & 17.51 & 17.63 & 0.67 & 22.87 & 11.94 & 10.9 \\
\hline$\rho\left(\mathrm{mg} / \mathrm{mm}^{3}\right)$ & & & & & & 1.67 & 1.67 & \\
\hline \multicolumn{9}{|l|}{ PAX21 } \\
\hline$\# 1$ & 33.02 & 22.29 & 10.73 & 9.70 & 9.60 & 20.25 & 17.64 & 2.6 \\
\hline$\# 2$ & 16 & 10.38 & 5.62 & 5.25 & 6.58 & 10.14 & 7.74 & 2.4 \\
\hline \#3 & 15.77 & 10.95 & 4.82 & 4.52 & 6.22 & 10 & 7.99 & 2.0 \\
\hline$\# 4$ & 30.75 & 22.24 & 8.51 & 8.40 & 1.29 & 20.21 & 16.24 & 3.97 \\
\hline B & 7.95 & & & & & 4.98 & & \\
\hline$C$ & 82.08 & & & & & 52.6 & & \\
\hline$\rho\left(\mathrm{mg} / \mathrm{mm}^{3}\right)$ & & & & & & 1.51 & 1.32 & \\
\hline
\end{tabular}


Figure 11. Measurements made possible by $\mu \mathrm{CT}$ : (a) volume of particles and crystal constituents in IM particles and (b) Density calculated from the mass and $\mu \mathrm{CT}$ volume measurements.
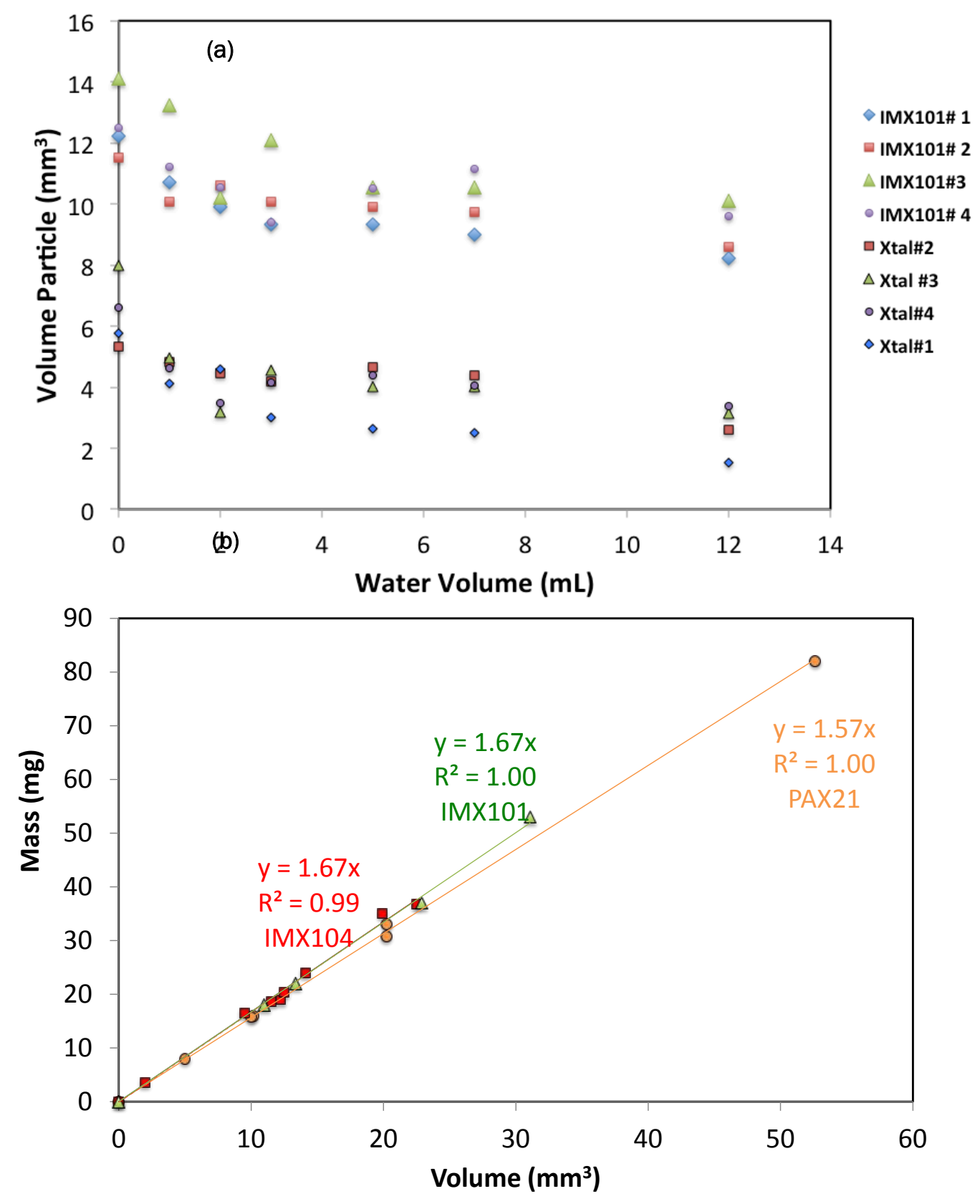

\subsubsection{Dissolution drip tests}

We used well-controlled laboratory drip experiments to measure mass dissolved as a function of water volume for detonated particles of IMX101 and IMX104. These particles were dissolved completely. 
Figure 12 shows results for the drip tests on four IMX101 particles (mass of $\mathrm{A}>\mathrm{B}>\mathrm{C}>\mathrm{D}$ ). To show the compositional variation among particles, we plotted the ratio of each compound measured to its initial mass (estimated based on the mass of the particle and the putative bulk composition) versus the cumulative water volume. The smallest particle $\mathrm{D}$, for example, has more NQ and less NTO and DNAN than IMX101 bulk composition, whereas particle C has more NTO and less NQ than the bulk. The sum of the dissolved components was, nevertheless, close to the mass of the original particle (Table 4).

Figure 12. Percent of the expected mass dissolved for compounds in IMX101 versus water volume $(\mathrm{mL})$.
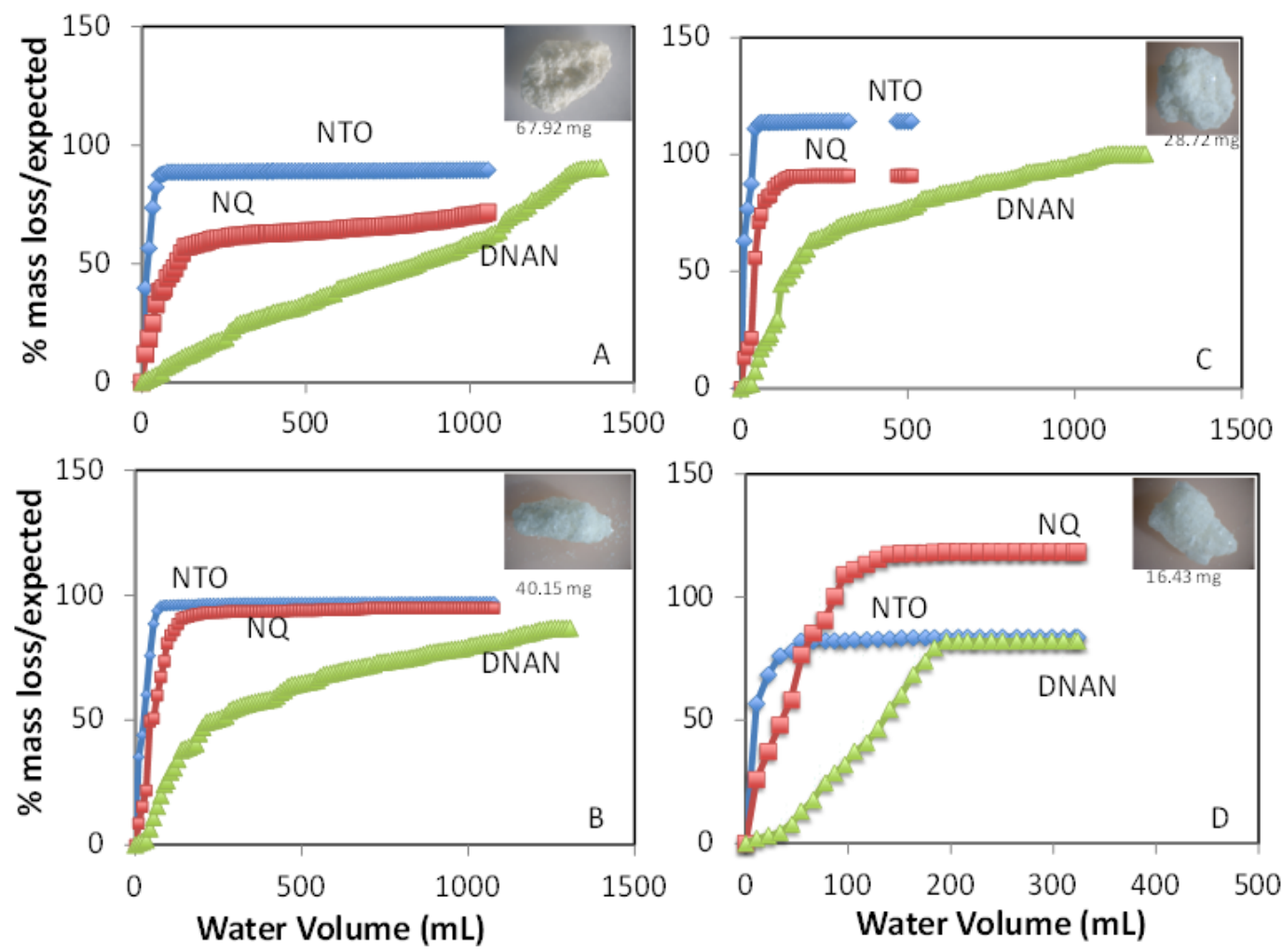
Table 4. Mass balance for IMX101 and IMX104 drip dissolution tests. The expected mass was calculated based on the initial mass of the particle and the putative compositions of the formulations.

\begin{tabular}{|c|c|c|c|c|c|c|c|c|}
\hline \multicolumn{5}{|c|}{ IMX101 } & \multicolumn{4}{|c|}{ IMX104 } \\
\hline & $\begin{array}{l}\text { NTO } \\
\text { (mg) }\end{array}$ & $\begin{array}{c}\mathrm{NQ} \\
(\mathrm{mg})\end{array}$ & $\begin{array}{l}\text { DNAN } \\
\text { (mg) }\end{array}$ & $\begin{array}{l}\text { Total } \\
\text { (mg) }\end{array}$ & $\begin{array}{l}\text { NTO } \\
\text { (mg) }\end{array}$ & $\begin{array}{c}\text { DNAN } \\
\text { (mg) }\end{array}$ & $\begin{array}{l}\text { RDX } \\
\text { (mg) }\end{array}$ & $\begin{array}{l}\text { Total } \\
\text { (mg) }\end{array}$ \\
\hline \multicolumn{5}{|l|}{$A$} & \multicolumn{4}{|l|}{1} \\
\hline expected & 13.58 & 25.13 & 29.21 & 67.92 & 5.16 & 3.12 & 1.46 & 9.74 \\
\hline measured & 12.26 & 20.69 & 26.8 & 59.76 & 5.55 & 2.95 & 1.67 & 10.17 \\
\hline$\%$ error & & & & 12 & & & & 4 \\
\hline \multicolumn{5}{|l|}{$B$} & \multicolumn{4}{|l|}{2} \\
\hline expected & 8.03 & 14.86 & 17.26 & 40.15 & 15.07 & 9.10 & 4.27 & 28.44 \\
\hline measured & 7.79 & 13.94 & 14.90 & 36.63 & 15.50 & 8.32 & 4.42 & 28.24 \\
\hline$\%$ error & & & & 9 & & & & 1 \\
\hline \multicolumn{5}{|l|}{ C } & \multicolumn{4}{|l|}{3} \\
\hline expected & 5.74 & 10.63 & 12.35 & 28.72 & 13.84 & 8.36 & 3.92 & 26.11 \\
\hline measured & 6.56 & 9.65 & 12.39 & 28.60 & 14.18 & 7.84 & 4.07 & 26.09 \\
\hline$\%$ error & & & & 0 & & & & 0 \\
\hline \multicolumn{5}{|l|}{ D } & \multicolumn{4}{|l|}{4} \\
\hline expected & 3.29 & 6.08 & 7.06 & 16.43 & 13.95 & 8.42 & 3.95 & 26.32 \\
\hline measured & 2.75 & 7.20 & 5.80 & 15.75 & 13.73 & 7.93 & 4.05 & 27.18 \\
\hline$\%$ error & & & & 4 & & & & 3 \\
\hline
\end{tabular}

These plots show that NTO is the first phase dissolved, followed by NQ, and that DNAN is last component dissolved, consistent with the results described above and with the solubility of each component (Table 1). The shape of the mass loss curves is also informative. NTO is lost quickly as evident by a steep rise in mass loss followed by a flat plateau. NQ has a less steep initial rise but also reaches a plateau, although for the largest particle, A, this occurs near the end of the test. The DNAN mass loss curves are quasi linear for $\mathrm{A}$ and $\mathrm{D}$ and are described by two lines of different slope for particles B and C. For particles B, C, and D, the steepest section of the DNAN mass loss curve occurs before NQ has completely dissolved. The dissolution of DNAN tracks that of NQ in this region, suggesting that NQ is controlling its dissolution. After NQ is completely dissolved, DNAN dissolves at a slower rate. We think that NTO dissolves independently and that the dissolution of NTO and NQ leave holes in the particle that increase DNAN's surface area and its dissolution. As soon as NQ has dissolved, the DNAN dissolves independently. 
The mass balances for these tests are good for particles $C$ and $D$ and are not as good for the two larger particles, A and B (Table 4). We suspect particle $B$ was lost close to the end of the test because the funnel holding particle B overflowed after $1249 \mathrm{~mL}$ of effluent had been collected (day 115) after which the concentration for DNAN was below detection. Although only a small volume of water overflowed, we suspect the particle was floating on the surface (these IM particles can trap air in their porous structures) and was lost along with the water. For particle A, however, the funnel did not overflow; and we can see that the concentration of DNAN in the water samples decreased gradually until it was below the detection limit. In this case, it is possible that a small piece broke from the particle between weighing the sample and placing it in the frit or that the particle contained small pieces of metal from the detonation that added to its mass but not to its dissolved mass.

Figure 13 shows results for the drip tests on four IMX104 particles (mass of $2>4>3>1$ ). Here again, NTO dissolved rapidly and independently of the DNAN-RDX matrix. The percent mass loss curves for the DNANRDX matrix are fairly linear with a larger percentage of DNAN dissolving early in the test and a larger fraction of RDX and HMX dissolving later in the test. Here we think that the RDX crystals are controlling the late stage dissolution of the particle and that the RDX needs to either dissolve or fall off to allow the underlying DNAN to dissolve. This process is similar to what occurs in Comp B (Lever et al. 2005).

Table 4 shows the mass balances for IMX104 tests. In all cases, the relative error between the initial mass of the particle and the mass recovered from the water samples was below $5 \%$. These laboratory drip tests indicate that the formulations are not being significantly photo- or bio-transformed because most of the mass (100 $\pm 5 \%)$ is recovered. If these IM formulations were transforming into new compounds the mass balance would be lower.

Although PAX21 is no longer being manufactured and used in munitions, for completeness, we dripped water on one PAX21 particle. Figure 14 shows the mass loss as a function of water volume. The $\mu \mathrm{CT}$ data do not show interconnections between the AP crystals and the surface, yet the data show that all of the AP dissolved in the first $12 \mathrm{~mL}$ of water. This finding indicates that water was able to access the interior of this $51 \mathrm{mg}$ particle. The data clearly show that the AP in PAX21 dissolves quickly and independently of the DNAN-RDX matrix. The PAX21 matrix has more RDX, 
with a 2:1 RDX:DNAN ratio, than does the IMX104 matrix, with a 1:1 RDX:DNAN ratio. Consequently, PAX21 particles may take longer to dissolve than comparable IMX104 particles.

Figure 13. Percent of the expected mass dissolved for compounds in IMX104 versus water volume $(\mathrm{mL})$.
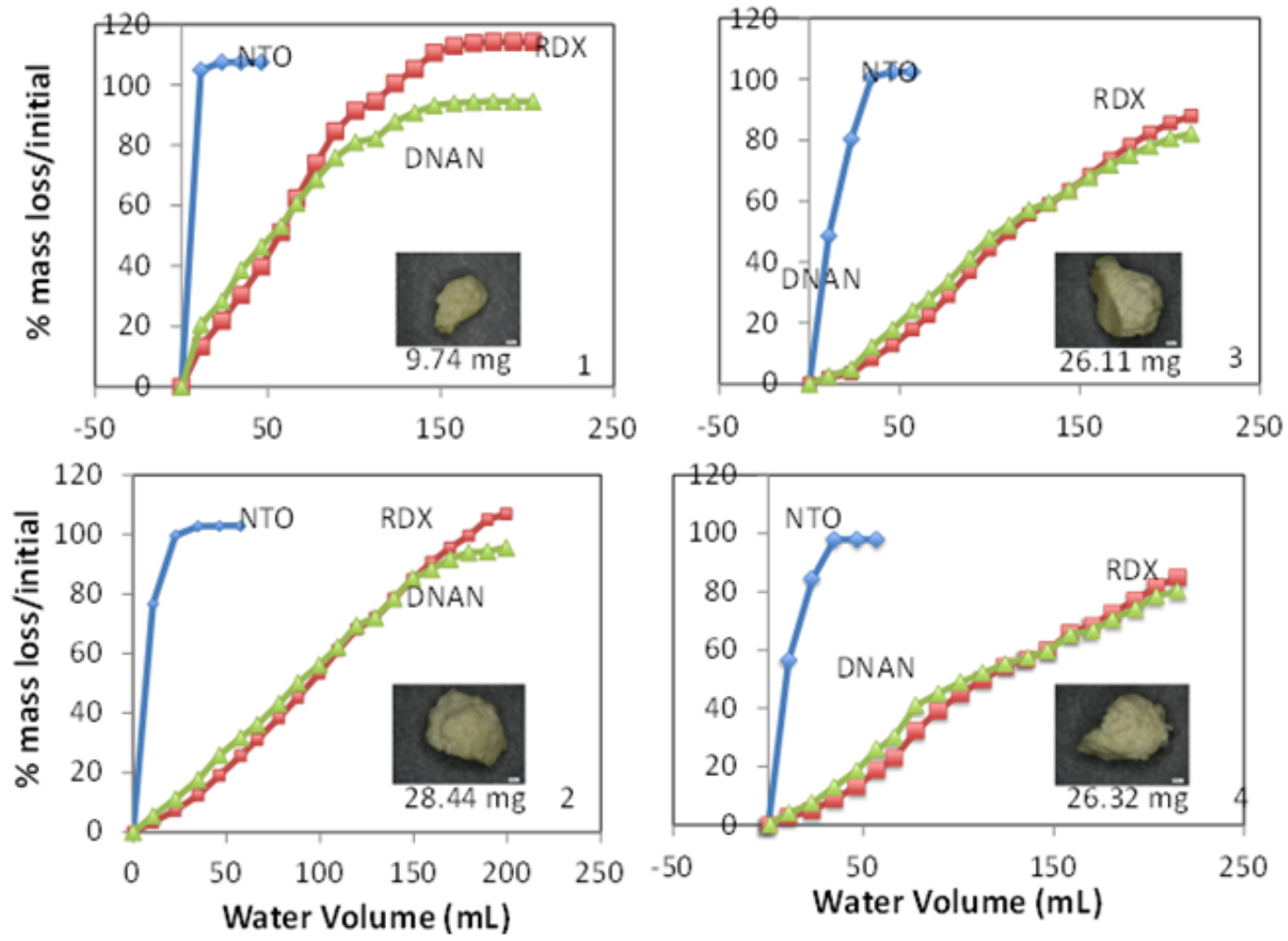
Figure 14. Drip dissolution test of a $51 \mathrm{mg}$ PAX21 particle.

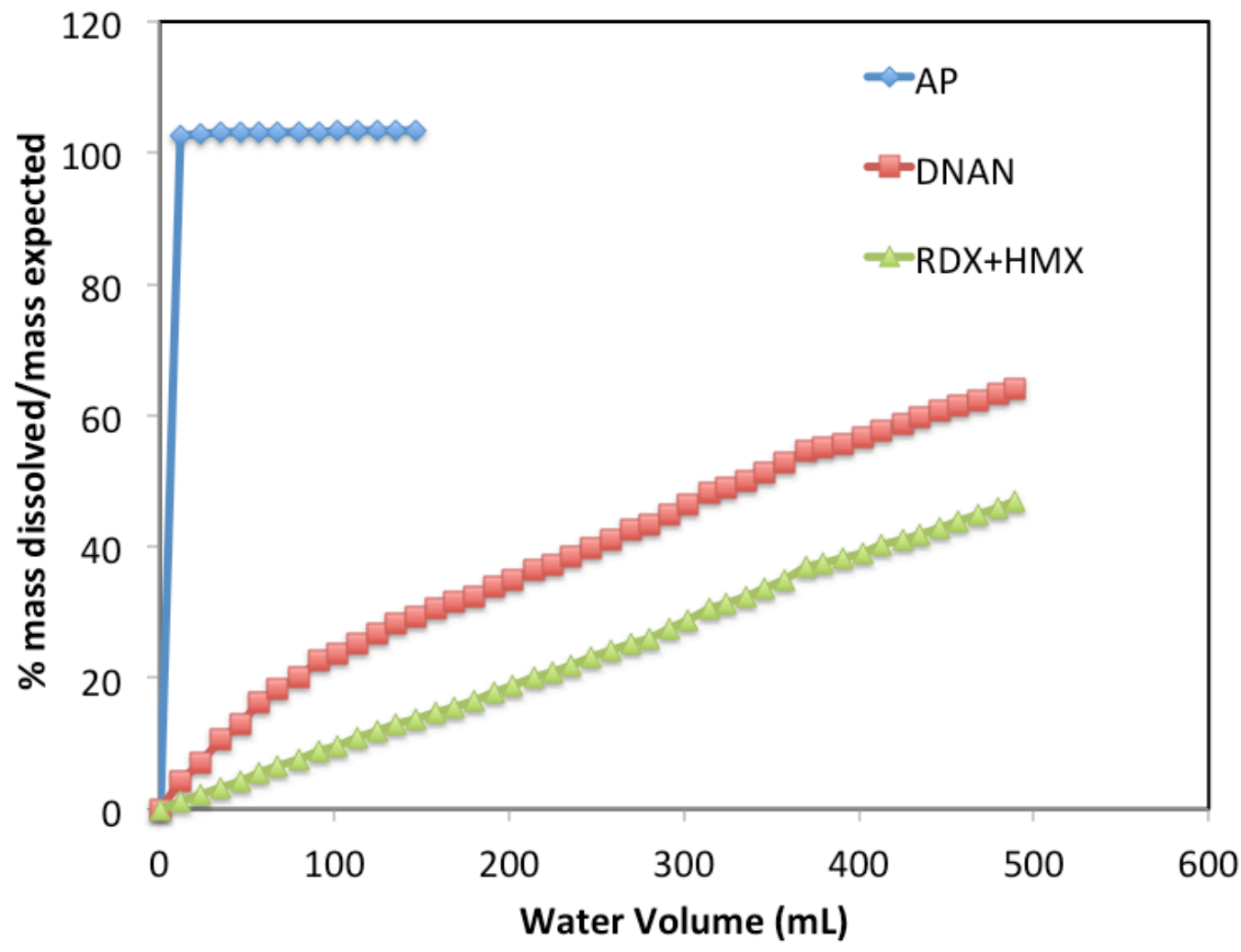

\subsubsection{Outdoor dissolution tests}

We set up the outdoor dissolution tests on $10 \mathrm{~J}$ une 2013, and they are still ongoing. We have collected water samples on eight separate occasions and analyzed six sets of water samples (Figure 15). A sum of the dissolved compound masses shows that the IMX101 chunks have lost between $21 \%$ to $65 \%$ of their masses, the IMX104 chunks between $33 \%$ and $80 \%$, and the PAX21 between $45 \%$ and $71 \%$ of their masses. We also find that these formulations are much more friable than chunks of high energetics. In less than 6 months, 8 of the 12 IM chunks have split; and all of the chunks have shed millimeter-sized particles (Figure 16a, b, and c). The average volume of rainfall interacting with these chunks was $739 \pm 82 \mathrm{~mL}$. For comparison, of the 12 chunks of TNT, 12 chunks of Comp B, 5 chunks of tritonal, and 5 chunks of $\mathrm{C} 4$ (Composition 4, a common variety of the plastic explosive known as " $\mathrm{C}$ ") placed outdoors to weather and dissolve, 4 of the chunks split, 14 spalled millimeter-sized particles, and 9 developed cracks (Taylor et al. 2010) in three years. 
Figure 15. Percent mass dissolved of the mass expected for the compounds in 5 chunks of IMX101, 5 chunks of IMX104, and 2 chunks of PAX21 set outside to weather and dissolve.
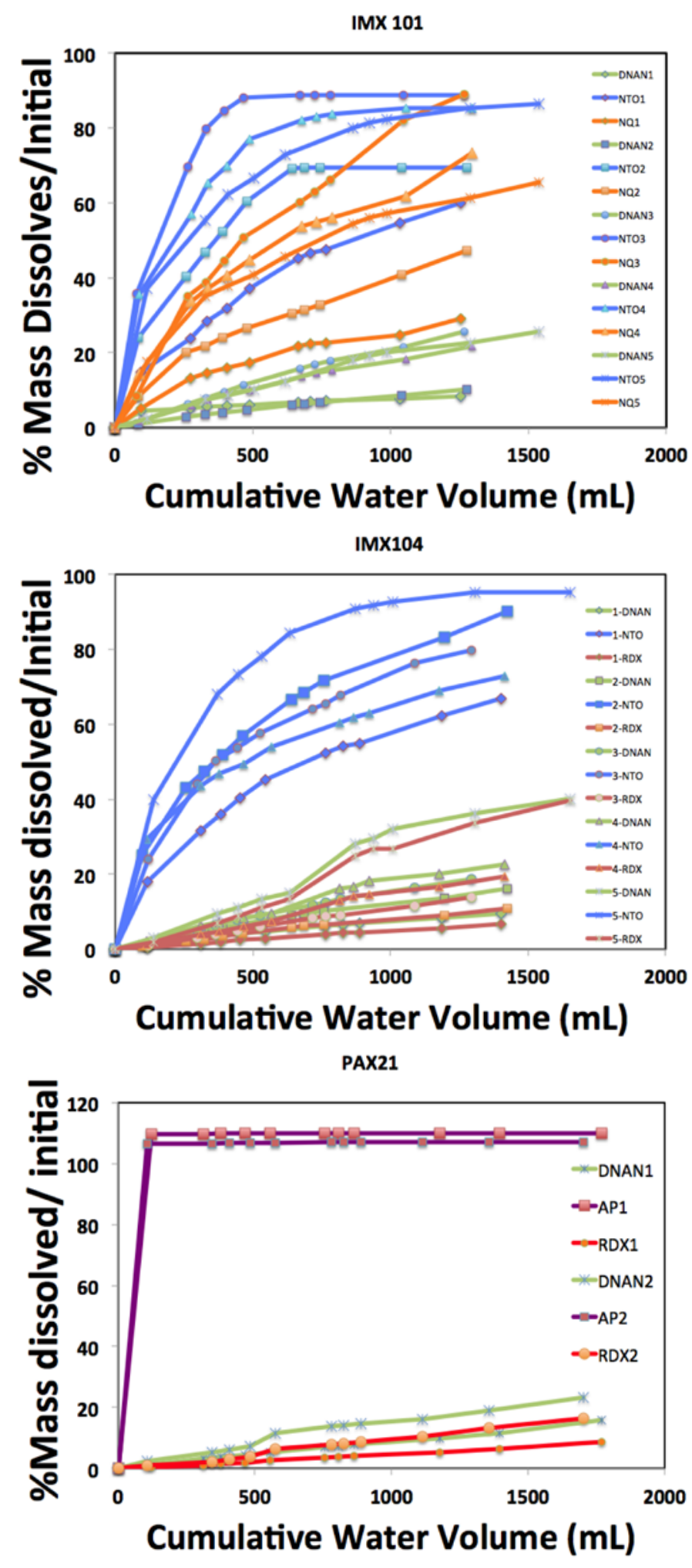
Figure 16. Sequential images of IMX101 particle \#1 and IMX104 particle \#3.

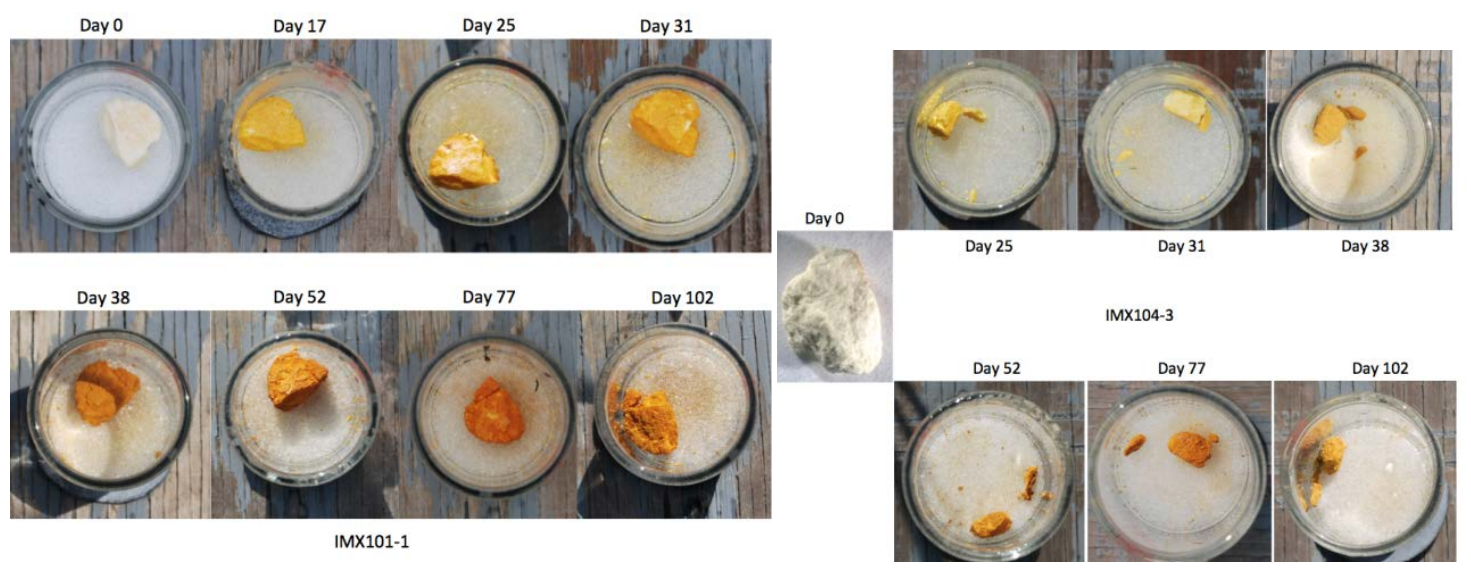

Our tests suggest that phototransformation of their surfaces is occurring because (1) the IM pieces changed color from cream or white to orange or brick red, (2) an unknown peak develops in the HPLC where polar substances elute, (3) this peak is largest in samples collected during the summer months when sun irradiance is highest, and (4) indoor (no sunlight) tests on IM explosives showed good mass balance (100 $\pm 5 \%)$ and no unaccounted for HPLC peaks. Although we have not yet calculated a mass balance for these samples (this will be done at the end of the test) we expect that it will be low because of the transformation of the IM compounds into unknown products that we are not analyzing for.

\subsection{4 pH of the IM solutions}

NTO is known to be acidic in solution (Chipen et al. 1966; Le Campion et al. 1997), and we measured the $\mathrm{pH}$ of the both the drip and the outdoor water samples to quantify how this changed during dissolution of the particle. For the IMX101 drip samples, we measured $\mathrm{pH}$ values initially in the low 3 range that increased to neutral as the NTO concentration decreased to $10 \mathrm{mg} \mathrm{L}^{-1}$ (Figure 17). The $\mathrm{pH}$ of the IMX104 samples showed a similar trend with the difference being that, because IMX104 contains proportionally more NTO than does IMX101, the concentrations were higher and the $\mathrm{pH}$ levels lower for the first few IMX104 samples. Figure 13 shows that there is a good correlation between NTO concentration and $\mathrm{pH}$ values between 2 and 5 for all samples. At NTO concentrations below $20 \mathrm{mg} \mathrm{L}^{-1}$, the $\mathrm{pH}$ can vary between 4 and 6; and at NTO concentrations below $10 \mathrm{mg} \mathrm{L}^{-1}$, the $\mathrm{pH}$ of the solution is close to neutral. The color of the solution was also found to correlate with the NTO concentration - the most yellow colored solutions had the highest NTO concentrations and the lowest $\mathrm{pH}$ values. 
Figure 17. Plot showing the relationship between NTO concentration and $\mathrm{pH}$ for indoor drip samples and outdoor samples. These data look similar to a titration curve.

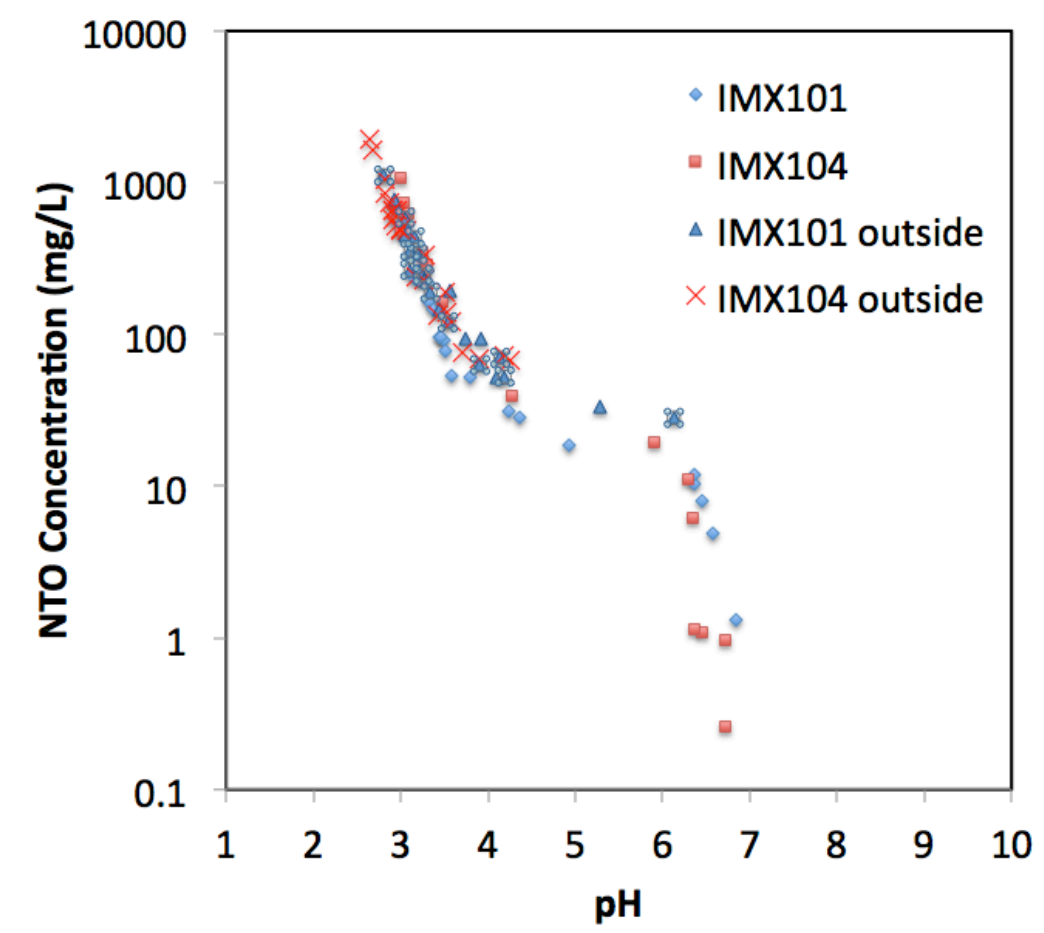




\section{Phototransformation studies}

\subsection{Methods}

\subsubsection{Phototransformation of NTO and DNAN in solution}

To evaluate direct phototransformation rates in aqueous solutions of NTO and DNAN, we followed ASTM Method E 896-92 (Standard Test Method for Conducting Aqueous Direct Photolysis Tests) (ASTM International 2005). We exposed solutions of NTO and DNAN to the simulated sunlight and quantified the concentration changes using an HPLC with UV/Vis detector. Specifically, we dissolved a sample in deionized water, adjusted to $\mathrm{pH} 7$ using a phosphate buffer, to achieve a $1 \mathrm{mg} \mathrm{L}^{-1}$ concentration and placed it in quartz NMR $5 \mathrm{~mm}$ tubes. We conducted phototransformation measurements by using a solar simulator, SUNTEST CPS+Xenon Test Instrument by Atlas Material Testing Technology (Chicago, IL) (Figure 18). The Atlas Suntest CPS+ uses an air-cooled xenon lamp to simulate UV and visible solar radiation in the $300-800 \mathrm{~nm}$ range. Irradiance (the radiative flux, or the amount of light incident on a unit area of a surface) of 765 $\mathrm{W} / \mathrm{m}^{2}$ was used for a period of 200-500 min with samples collected and analyzed over time.

Figure 18. SUNTEST CPS+ Xenon Test Instrument used to evaluate phototransformation of NTO and DNAN in solution.

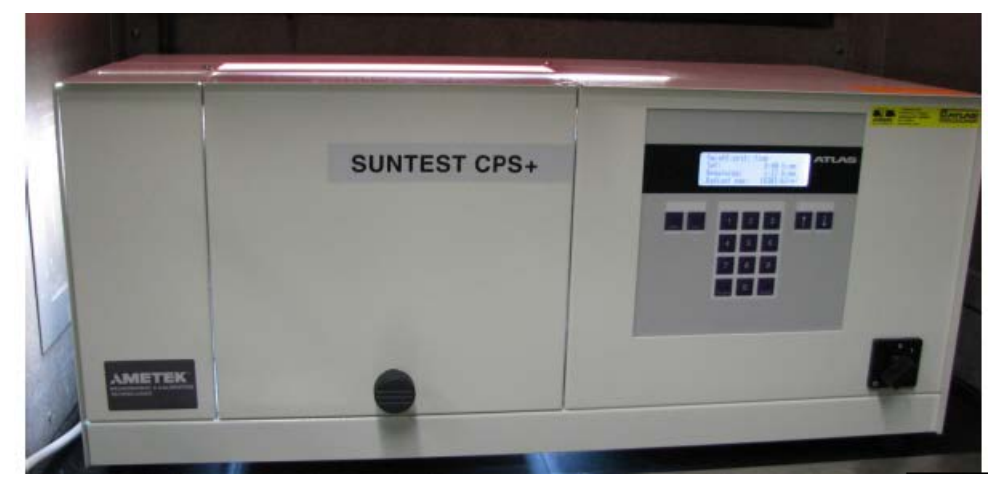

We measured concentrations of DNAN and NTO in solution before and after irradiation by using an Agilent 1200 series HPLC with C18 column at $30^{\circ} \mathrm{C}$, a $10 \mu \mathrm{L}$ manual injection loop, and acetonitrile/ water (60/40) mobile phase. A UV/Vis detector measured DNAN at $300 \mathrm{~nm}$ (using $360 \mathrm{~nm}$ as a reference) and NTO at $375 \mathrm{~nm}$ (using $390 \mathrm{~nm}$ as a reference). No analysis of phototransformation products was done during 
phototransformation rate studies. Dark samples of DNAN and NTO were monitored as the control. We found both compounds to be stable in the dark over the time of the experiment.

According to ASTM 896 (ASTM International 2005), phototransformation of ionizable materials can exhibit marked $\mathrm{pH}$ effects that are attributable to changes in speciation. Therefore, if different species are present in the $\mathrm{pH}$ 5- 9 range, testing should be conducted at $2-3 \mathrm{pH}$ values throughout the pH 5- 9 range. NTO is an ionizable compound (Figure 19) with a $\mathrm{pK}_{\mathrm{a}}$ of 3.67 (Kofman et al. 1980). A pH of $1 \mathrm{ppm}$ NTO solution in deionized water equals to a $\mathrm{pH}$ of 4.98 . Therefore, in this study, direct phototransformation of NTO was measured at pH 1.5, 4.2, 7.0, and 8.8. We achieved these $\mathrm{pH}$ values by using phosphate, acetate, phosphate, and borate buffers, respectively.

Figure 19. Ionized and neutral forms of NTO (Smith and Cliff 1999).
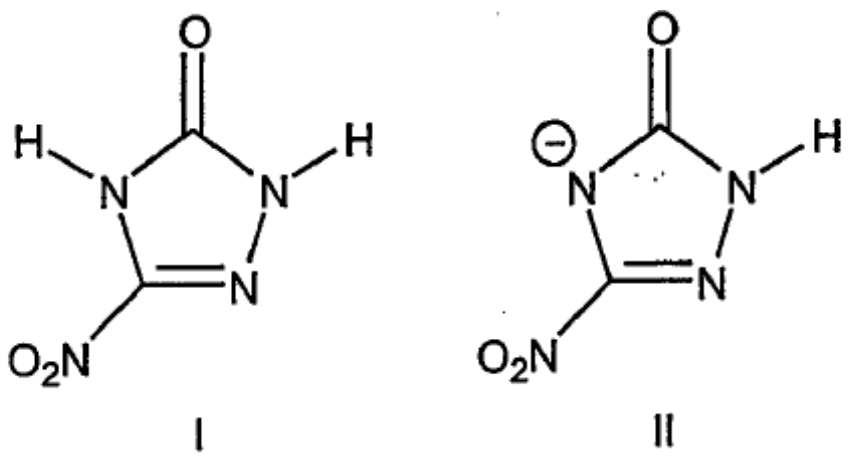

In addition to direct phototransformation, we measured the indirect phototransformation of neat DNAN and NTO in solution. To determine the contribution of dissolved natural organic matter present in solution to the transformation of IM compounds under light, we used U.S. EPA method OPPTS 835.5270, "Indirect Photolysis Screening Test" (U.S. EPA 1998). Humic acid (from Sigma Aldrich, St. Louis, MO) in 60 ppm dissolved OC concentration served as natural organic matter.

We took initial phototransformation rate measurements (including those at varying $\mathrm{pH}$ for $\mathrm{NTO}$ ) at $35^{\circ} \mathrm{C}$ because it is the lowest temperature that the solar simulator can maintain. This temperature is within the recommended standard range of test temperatures for phototransformation according to ASTM E896-92 (ASTM International 2005) but is higher than can be expected in some environments. Consequently, to describe temperature dependence of phototransformation rates, we performed direct 
phototransformation measurements at $\mathrm{pH} 7$ for four different temperatures between $70^{\circ} \mathrm{C}$ and $35^{\circ} \mathrm{C}$. Control solutions set in the dark indicated that both compounds were stable across the temperature range used. Once we obtained rates at different temperatures, we used the following equation to relate measured reaction rates to the activation energy of the reaction:

$$
k_{\text {photo }}=A e^{-\frac{E_{a}}{R T}}
$$

where reaction rate, $\mathrm{k}_{\text {photo, }}$ is a function of temperature, $\mathrm{T}$, and activation energy of the reaction, $\mathrm{E}_{\mathrm{a}}$.

Calculated activation energy of the phototransformation of each compound allows us to determine the rate reaction at any temperature. The HYDRUS model is able to incorporate information about the energy of activation $\left(E_{a}\right)$ for the phototransformation reactions for DNAN and NTO into predictions of IM fate in the environment. We did all measurements in triplicate and observed them to be highly reproducible with error generally smaller than the size of the symbols on the plot.

\subsection{Results}

\subsubsection{Direct and indirect phototransformation of NTO}

Figure 20 presents NTO phototransformation as a function of time. Measured NTO concentrations were highly reproducible with standard deviation of the mean less than $1 \%$. All phototransformation reactions exhibited strong first-order kinetics (Figure 20). The transformation rate constants were estimated by fitting regression lines to plots of the logarithm of NTO concentration as a function of time. The regression lines have high correlation coefficients $\left(\mathrm{R}^{2}=0.99\right)$, indicating a good fit and high confidence in the estimated rate constants.

The $\mathrm{pH}$ of the solution strongly affected the phototransformation of NTO, with rates being highest at extreme $\mathrm{pH}$ values and lowest at neutral $\mathrm{pH}$ (Figure 20a; Table 5). The rate constant at $\mathrm{pH} 7$ was estimated to be equal to $0.0014 \mathrm{~min}^{-1}$ or $0.084 \mathrm{hr}^{-1}$, resulting in a half-life of $495 \mathrm{~min}$ or $8.3 \mathrm{hr}$. Measured NTO phototransformation rate constants were higher than constants measured previously for RDX (Bordeleau et al. 2013), which NTO is replacing in explosive formulations. 
Figure 20. Phototransformation of NTO as affected by (a) solution pH and (b) the presence of natural organic matter.
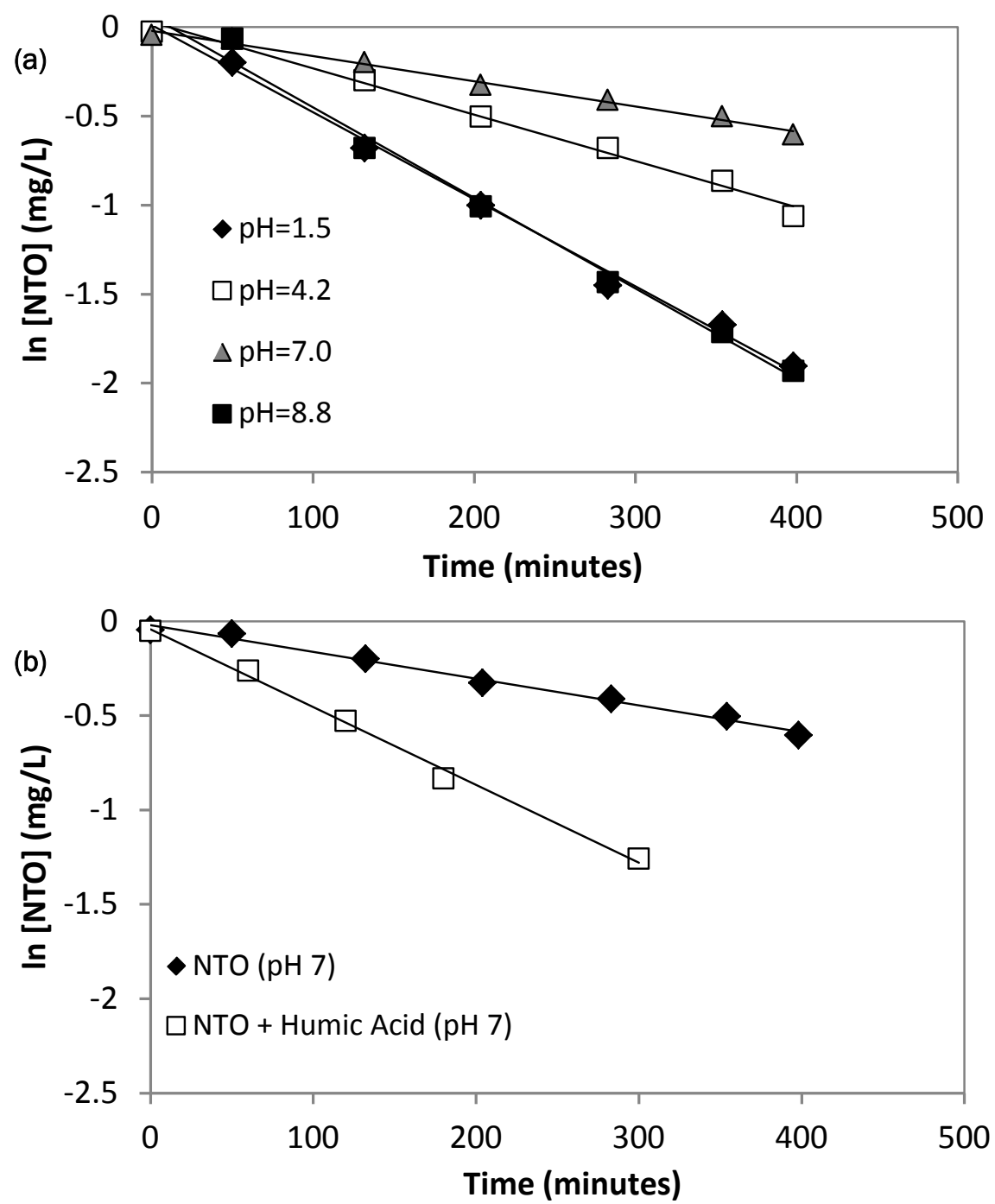

NTO phototransformation was strongly affected by the presence of natural organic matter (humic acids) with the phototransformation rate being almost three times higher in the presence of organic matter compared to rates measured when no organic matter was present (Figure 20b, Table 5).

\subsubsection{Direct and indirect phototransformation of DNAN}

DNAN also experienced first-order kinetics of phototransformation (Figure 21). At pH 7, the phototransformation rate constant was 0.0021 min $^{-1}$, resulting in a half-life of $330 \mathrm{~min}$. For comparison, the half-life of TNT in pure water is $700 \mathrm{~min}$ and $200 \mathrm{~min}$ in fresh water. The shorter half-life of fresh water may be due to the presence of natural organic matter (O'Sullivan et al. 2011; Prak and O'Sullivan 2012). Unlike TNT and 
NTO, which have been shown to be affected by organic matter in solution, DNAN's phototransformation was not influenced by the presence of organic matter, with a phototransformation rate of $0.0020 \mathrm{~min}^{-1}$ and a half life of $346 \mathrm{~min}$, values that were not significantly different from ones measured for DNAN in water without organics present.

Figure 21. Phototransformation rate of DNAN in water and in water with humic acid. Error bars are smaller than the symbols.

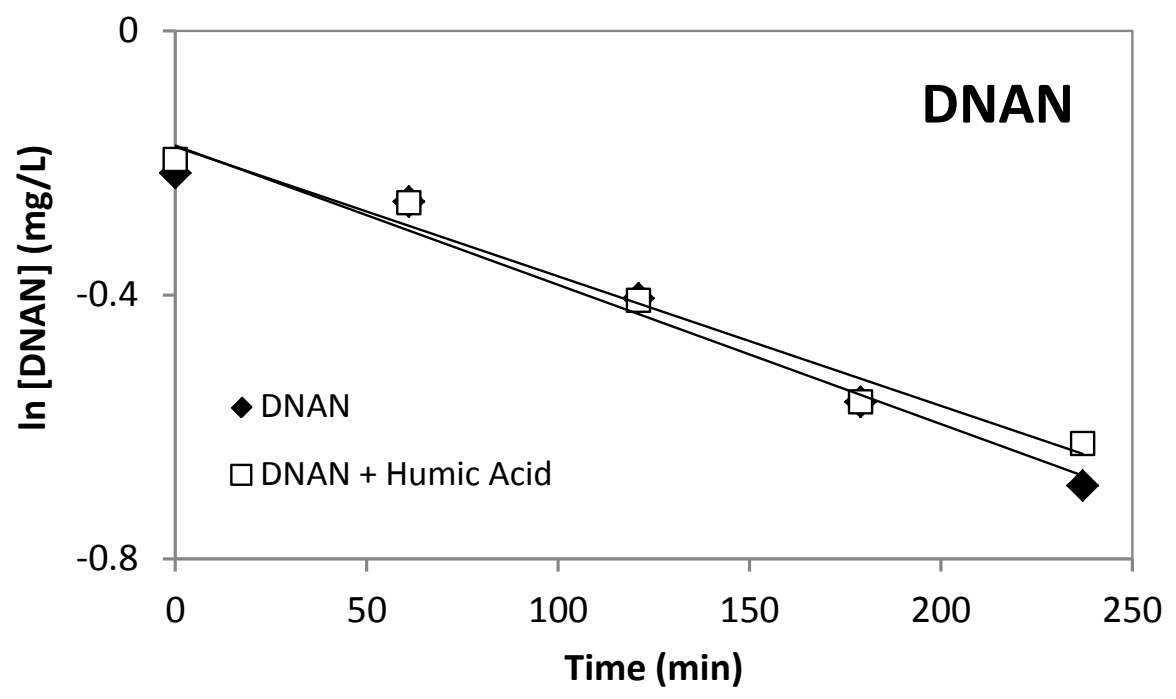

Measured DNAN phototransformation rate constants were similar to ones determined in Rao et al. (2013), which reported an average half-life for DNAN of 0.70 days with values ranging between 0.11 and 1.51 days. The range in values depended on the wavelength and the intensity of the light source, the initial DNAN concentration, and the temperature. For conditions similar to ones used in our study, Rao et al. (2013) estimated a 0.25 day half life.

Table 5. Determined phototransformation rate constants, $k_{\text {photo, }}$ and half-lives, $t_{3 / 2}$, for NTO and DNAN measured at $35^{\circ} \mathrm{C}, 765 \mathrm{Watts} / \mathrm{m}^{2}$.

\begin{tabular}{|l|c|c|c|c|c|c|c|c|c|c|}
\hline & \multicolumn{9}{|c|}{ NTO } & \multicolumn{7}{c|}{ DNAN } \\
\hline \multicolumn{1}{|c|}{$\mathrm{pH}$} & $\begin{array}{c}k_{\text {photo }} \\
\left(\mathrm{min}^{-1}\right)\end{array}$ & $\begin{array}{c}k_{\text {photo }} \\
\left(\mathrm{h}^{-1}\right)\end{array}$ & $\begin{array}{c}t_{3 / 2} \\
(\mathrm{~min})\end{array}$ & $\begin{array}{c}t_{3 / 2} \\
(\mathrm{~h})\end{array}$ & $\begin{array}{c}t_{3 / 2} \\
(\text { day })\end{array}$ & $\begin{array}{c}k_{\text {photo }} \\
\left(\mathrm{min}^{-1}\right)\end{array}$ & $\begin{array}{c}k_{\text {photo }} \\
\left(\mathrm{h}^{-1}\right)\end{array}$ & $\begin{array}{c}t_{3 / 2} \\
(\mathrm{~min})\end{array}$ & $\begin{array}{c}t_{3 / 2} \\
(\mathrm{~h})\end{array}$ & $\begin{array}{c}t_{3 / 2} \\
(\text { day })\end{array}$ \\
\hline Direct \\
\hline 1.5 & 0.0049 & 0.294 & 139 & 2.3 & 0.10 & & & & & \\
\hline 4.2 & 0.0026 & 0.156 & 267 & 4.5 & 0.19 & & & & & \\
\hline 7.0 & 0.0014 & 0.084 & 495 & 8.3 & 0.34 & 0.0021 & 0.126 & 330 & 5.5 & 0.23 \\
\hline 8.8 & 0.0051 & 0.306 & 138 & 2.3 & 0.10 & & & & & \\
\hline Indirect & & & & & & \\
\hline 7.0 & 0.0041 & 0.246 & 168 & 2.8 & 0.12 & 0.0020 & 0.120 & 346 & 5.8 & 0.24 \\
\hline
\end{tabular}




\subsubsection{Phototransformation of NTO and DNAN as a function of temperature}

In addition, we performed a set of experiments to determine the effect of temperature on phototransformation rate constants of NTO and DNAN and to calculate the $\mathrm{E}_{\mathrm{a}}$ of phototransformation reactions for these compounds. We found that phototransformation of NTO was relatively insensitive to temperature (Figures 22, 23, and 24; Table 6), with $\mathrm{t}_{1 / 2}$ equal to 0.30 days at $35^{\circ} \mathrm{C}$ and 0.27 days at $70^{\circ} \mathrm{C}$, resulting in an $\mathrm{E}_{\mathrm{a}}$ of $2.65 \mathrm{~kJ}$ $\mathrm{mol}^{-1}$ and a predicted half-life of 0.31 days at $27^{\circ} \mathrm{C}$. Low $\mathrm{E}_{\mathrm{a}}$ for NTO suggests catalysis by decomposition products.

Figure 22. Effect of temperature on DNAN and NTO phototransformation rates.
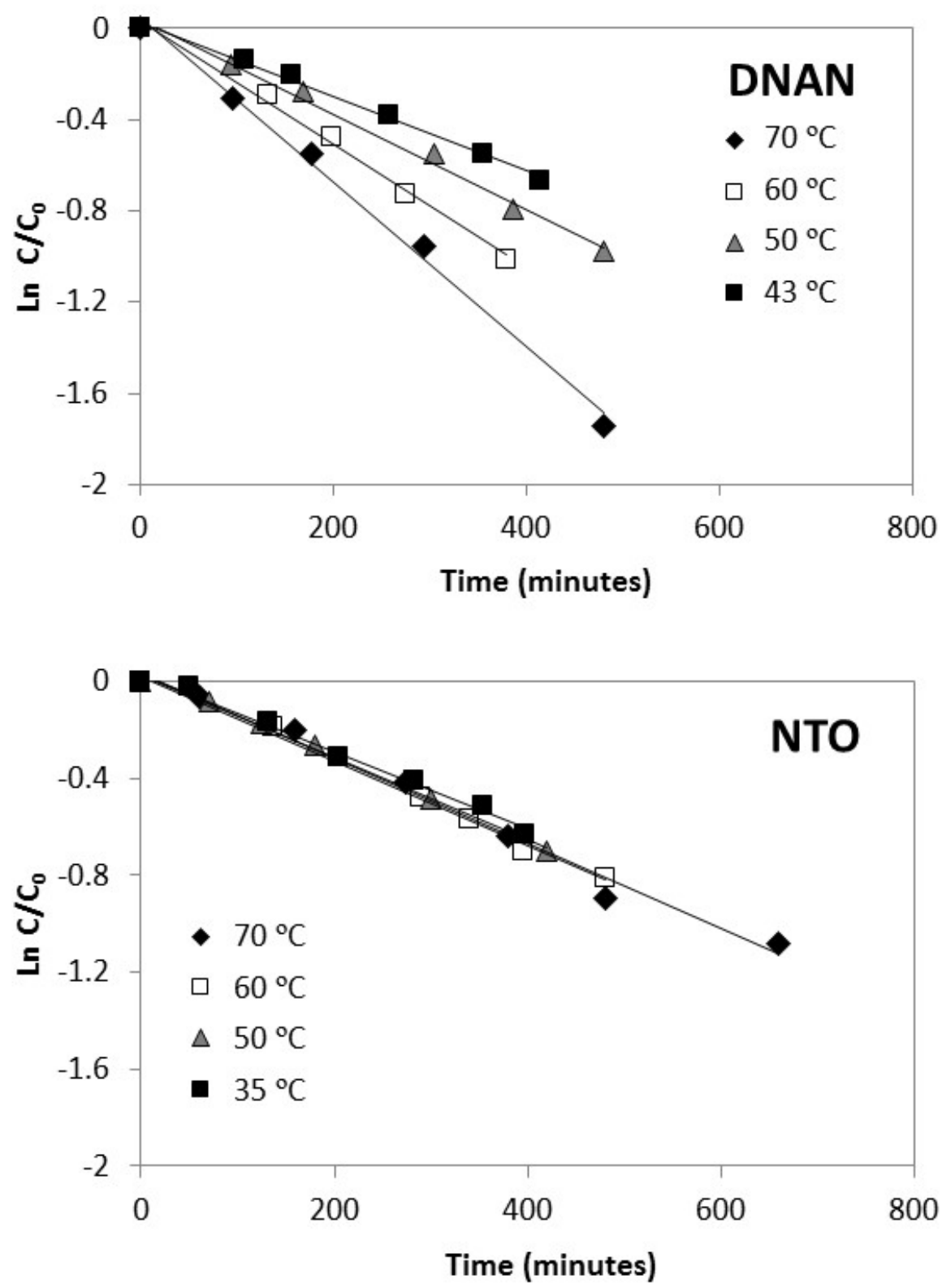
Figure 23. Natural logarithm of the phototransformation rate constant plotted as a function of temperature for DNAN and NTO.
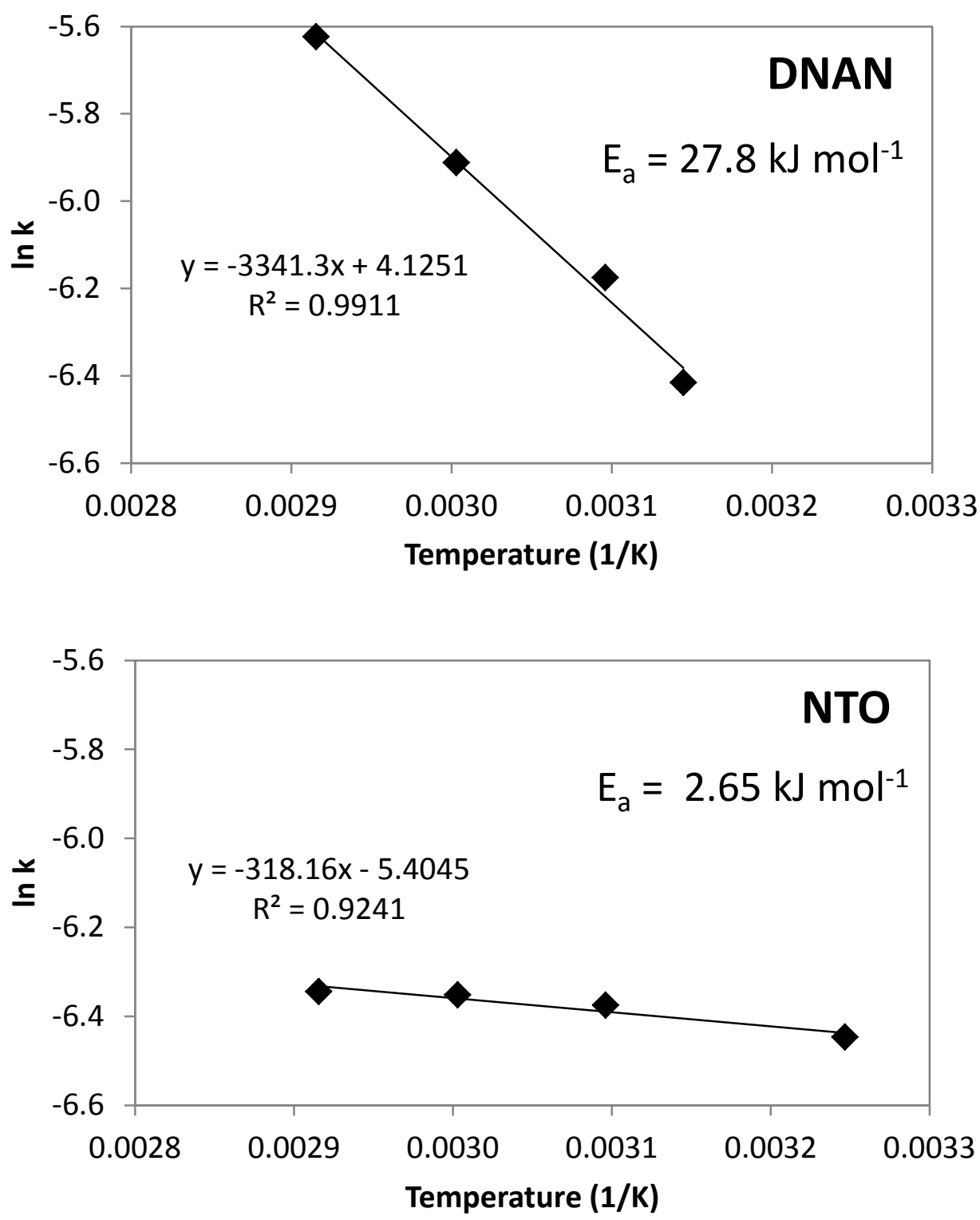

Phototransformation of DNAN was temperature dependent (Figure 22, 23, 24; Table 6) with $E_{a}=27.8 \mathrm{~kJ} \mathrm{~mol}^{-1}$; DNAN had a measured $\mathrm{t}_{1 / 2}$ of 0.13 days at $70^{\circ} \mathrm{C}, 0.23$ days at $35^{\circ} \mathrm{C}$, and a predicted half-life of 0.50 days at $27^{\circ} \mathrm{C}$. Determined activation energies for DNAN were lower than determined by Rao et al. (2013), which reported an $\mathrm{E}_{\mathrm{a}}$ of $53 \mathrm{~kJ}$ mol-1. Knowing the activation energies of the phototransformation reactions allows us to calculate the transformation rates for different environments. 
Figure 24. Measured and predicted values of NTO and DNAN half-lives as a function of temperature.

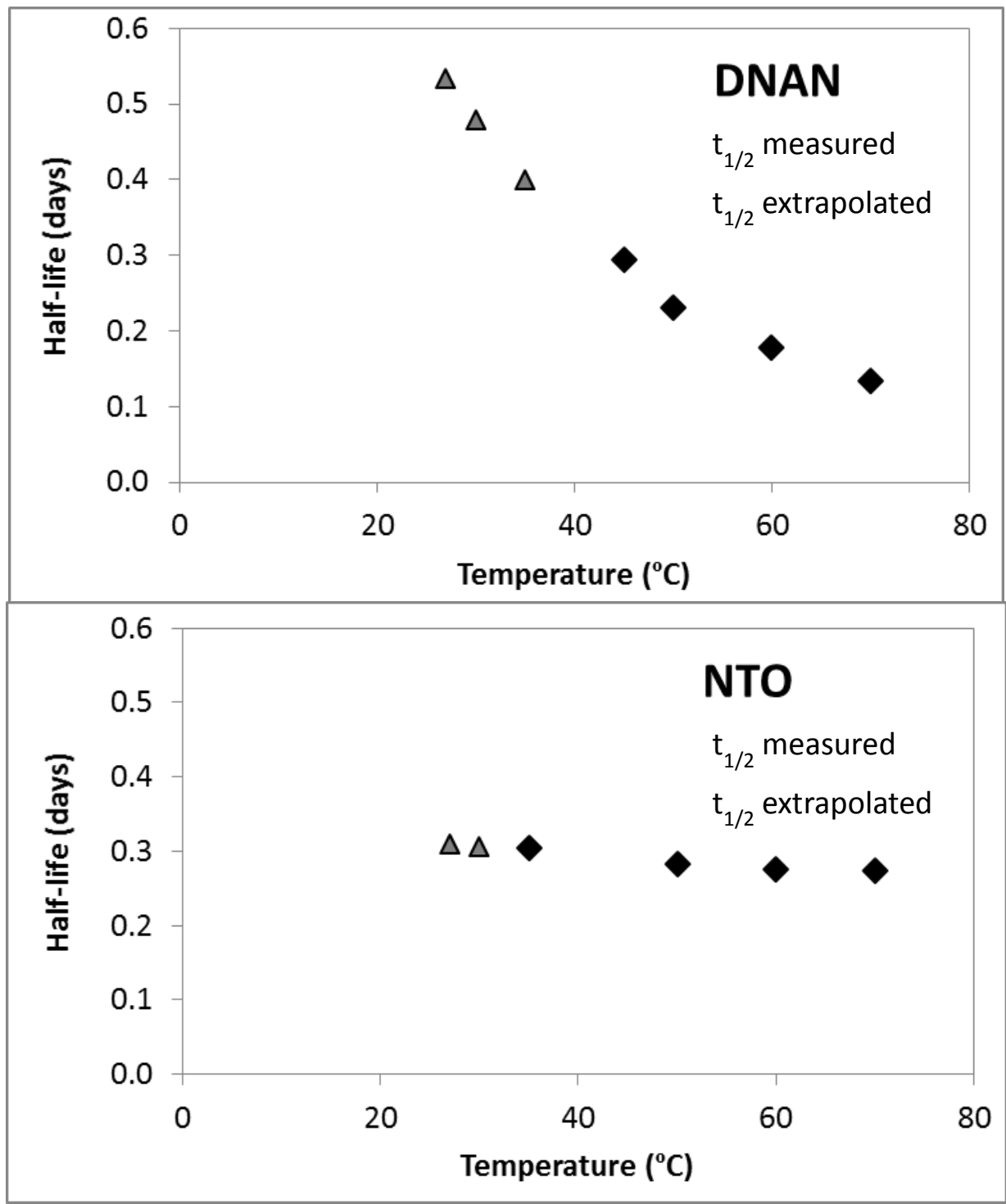

Table 6. Phototransformation rate constant, $k_{\text {photo, }}$ and half-life, $t_{32}$, of NTO and DNAN tabulated as a function of temperature. Measurements were made at $765 \mathrm{~W} / \mathrm{m}^{2}$ irradiance.

\begin{tabular}{|l|c|c|c|c|c|c|c|c|}
\hline & \multicolumn{5}{|c|}{ NTO } & \multicolumn{5}{c|}{ DNAN } \\
\hline Temperature & $\begin{array}{c}k_{\text {photo }} \\
\left(\mathrm{min}^{-1}\right)\end{array}$ & $\begin{array}{c}t_{1 / 2} \\
(\mathrm{~min})\end{array}$ & $\begin{array}{c}t_{1 / 2} \\
(\mathrm{~h})\end{array}$ & $\begin{array}{c}t_{1 / 2} \\
(\text { day })\end{array}$ & $\begin{array}{c}k_{\text {photo }} \\
\left(\mathrm{min}^{-1}\right)\end{array}$ & $\begin{array}{c}t_{1 / 2} \\
(\mathrm{~min})\end{array}$ & $\begin{array}{c}t_{1 / 2} \\
(\mathrm{~h})\end{array}$ & $\begin{array}{c}t_{1 / 2} \\
(\mathrm{day})\end{array}$ \\
\hline $27^{\circ} \mathrm{C}{ }^{*}$ & 0.00155 & 446 & 7.4 & 0.31 & 0.00091 & 763 & 12.7 & 0.53 \\
\hline $35^{\circ} \mathrm{C}$ & 0.00159 & 437 & 7.3 & 0.30 & 0.00120 & 576 & 9.6 & 0.40 \\
\hline $43^{\circ} \mathrm{C}$ & & & & & 0.00164 & 424 & 7.1 & 0.29 \\
\hline $50^{\circ} \mathrm{C}$ & 0.00170 & 407 & 6.8 & 0.28 & 0.00208 & 333 & 5.6 & 0.23 \\
\hline $60^{\circ} \mathrm{C}$ & 0.00174 & 398 & 6.6 & 0.28 & 0.00271 & 256 & 4.3 & 0.18 \\
\hline $70^{\circ} \mathrm{C}$ & 0.00176 & 395 & 6.6 & 0.27 & 0.00361 & 192 & 3.2 & 0.13 \\
\hline
\end{tabular}

*Extrapolated. 


\section{Soil interactions}

\subsection{Materials and methods}

\subsubsection{Soil sample collection and characterization}

To quantify IM- soil interactions, we used soils having a wide range of properties and that were collected on military installations located across the United States. The U.S. Army National Guard Environmental Directorate provided uncontaminated soil samples from a number of different locations. In addition, we used soils that were previously collected studied for their interaction with explosive and propellant constituents (Dontsova et al. 2006, 2007, 2009a, 2009b; Taylor et al. 2012). Using the same soils allows us to compare our results for IMs to those obtained for other energetics. We used only surface soils (top $30 \mathrm{~cm}$ ).

We air dried, sieved ( $<2 \mathrm{~mm}$ ), and characterized the collected soils prior to the experiments. We analyzed OC by dry combustion according to the U.S. EPA's standard operating procedure for sediments (U.S. EPA 2010). To ensure a complete removal of inorganic carbon, we treated a known mass of soil with $5 \%$ phosphoric acid $\left(\mathrm{H}_{3} \mathrm{PO}_{4}\right)$. Following acid pretreatment, we used a Shimadzu SSM-5000A Solid Sample Combustion Unit to analyze OC. A Beckman Coulter LS 13320 Laser Diffraction Particle Size Analyzer (by Beckman Coulter, Inc., Fullerton, CA) analyzed particle sizes. A Beckman Coulter SA 3100 measured specific surface area (SSA) by $\mathrm{N}_{2}$ adsorption, applying Brunauer-Emmett-Teller theory (Brunauer et al. 1938). We used a VWR sympHony SB70P Benchtop Digital $\mathrm{pH}$ and EC (electrical conductivity) Meter to test a 1:1 soil:solution mixture.

\subsubsection{IM compounds and their transformation products}

For the experiments, we obtained the technical grade NTO and DNAN from the U.S. Army Armament Research, Development and Engineering Center (ARDEC), Picatinny Arsenal. Calibration standards for NTO were prepared using the same NTO, while DNAN standards were prepared using DNAN (CAS: 119-27-7, 98\% purity) purchased from Alfa-Aesar (Ward Hill, MA). Known daughter compounds of DNAN (Perreault et al. 2011; Olivares et al. 2013), 2-methoxy-5-nitroaniline (MENA) (CAS: 99-59-2, 98\% purity) and 2,4-Diaminoanisole (CAS: 615-05-4 analytical standard), 
that we used to calibrate HPLC were obtained from Sigma-Aldrich (St. Louis, MO).

\subsubsection{Analytical methods}

We quantified NTO concentration by using a Dionex Ultimate 3000 HPLC equipped with a diode array detector (from ThermoFisher, MA). We adapted the running method from Le Campion et al. (1999a). The mobile phase, made from AcN with 0.1\% TFA and deionized water (75:25), was run isocractically at $1 \mathrm{~mL} \mathrm{~min}^{-1}$. Oven temperature was set at $32^{\circ} \mathrm{C}$. NTO and its transformation products were separated using a Thermo Scientific Hypercarb Column. We detected NTO at approximately $2.5 \mathrm{~min}$ by using a $315 \mathrm{~nm}$ wavelength. The UV detector was set at $220 \mathrm{~nm}$ to monitor for presence of ATO (Le Campion et al. 1998), but we did not detect ATO in any analyzed samples.

The parameters used for analyzing DNAN samples were the following: a mobile phase ratio of 43:57 methanol and water and flow rate of $1 \mathrm{~mL}$ $\mathrm{min}^{-1}$. The column used for analysis was the Thermo Scientific Acclaim reversed phase C-18 column. The wavelengths used were $300 \mathrm{~nm}$ for DNAN, $254 \mathrm{~nm}$ for MENA, and $210 \mathrm{~nm}$ for 2,4-diaminoanisole (Olivares et al. 2013).

\subsubsection{Batch soil adsorption studies}

To determine the fate of IM compounds in soils and the effect of soil heterogeneity on soil interactions with IM compounds, we conducted a series of kinetic and equilibrium batch soil sorption experiments (Roy et al. 1992). In these experiments, we mixed soils with solutions of NTO or DNAN and allowed them to equilibrate for a predetermined amount of time. We then separated the soil from the solution and analyzed the supernatant to determine the amount of NTO or DNAN remaining in solution. We conducted preliminary experiments to determine the optimal soil:solution ratios of NTO and DNAN (Table 7) and the effect of background salt solution on adsorption of these compounds. For follow-on studies, we selected tests that resulted in $70 \%-90 \%$ of the original NTO or DNAN amount remaining in the solution. The experiments used deionized water or $0.005 \mathrm{~mol} \mathrm{~L}^{-1} \mathrm{CaCl}_{2}$ solution to establish the effect of background salt solution on adsorption. 
To create a $1 \mathrm{mg} \mathrm{L}^{-1}$ input solution, we added $10 \mathrm{~mL}$ of a $100 \mathrm{mg} \mathrm{L}^{-1}$ stock solution of NTO to $990 \mathrm{~mL}$ of deionized water or of $0.005 \mathrm{~mol} \mathrm{~L}^{-1} \mathrm{CaCl}_{2}$ solution. We diluted DNAN further to $0.5 \mathrm{mg} \mathrm{L}^{-1}$. Soils were weighed into clean, labeled $30 \mathrm{~mL}$ borosilicate glass centrifuge tubes according to Table 7. We then pipetted $20 \mathrm{~mL}$ of $1 \mathrm{mg} \mathrm{L}^{-1} \mathrm{NTO}$ (or $0.5 \mathrm{mg} \mathrm{L}^{-1} \mathrm{DNAN}$ ) in water or $0.005 \mathrm{M} \mathrm{CaCl}_{2}$ solution into each centrifuge tube. An aliquot of the input solution was collected and stored to determine the initial concentration of the solute later. We conducted duplicate or triplicate tests, depending on observed variability, and prepared blanks by putting the input solution (prepared either in water or $0.005 \mathrm{M} \mathrm{CaCl}_{2}$ ) into a centrifuge tube holding no adsorbent. A reciprocating shaker agitated blanks and samples continuously at $110 \mathrm{rpm}$ for $24 \pm 0.5 \mathrm{hr}$. Experiments were conducted under constant conditions, away from light and at room temperature $\left(22 \pm 3^{\circ} \mathrm{C}\right)$.

After $24 \mathrm{hr}$ of agitation, we recorded any changes observed in the adsorbent or solutions and centrifuged the tubes for $20 \mathrm{~min}$ at $4700 \mathrm{rpm}$ (4816 relative centrifugal force). A minimum of $3 \mathrm{~mL}$ of supernatant was filtered through 0.45 $\mu \mathrm{m}$ Millex-HV PVDF filter (from EMD Millipore, Darmstadt, Germany) and placed in labeled $4 \mathrm{~mL}$ amber vials. For experiments with NTO, we pipetted $0.1 \mathrm{~mL}$ of this sample into labeled autosampler vials for analysis and diluted them to $1 \mathrm{~mL}$ with 75:25 AcN:deionized water mixture. We analyzed the supernatant solution for target compounds, NTO and NTO transformation product, ATO, by HPLC, and analyzed solutions containing DNAN by using HPLC directly after filtering without additional dilution. Section 4.1.3 of this report describes HPLC methods for NTO, DNAN, and their transformation products. The $\mathrm{pH}$ and EC of each sample was determined on an aliquot using a VWR SympHony SB70P Benchtop Digital pH and EC Meter (VWR, Radnor, PA). We wrapped all prepared solutions in aluminum foil (to prevent phototransformation) and sealed them with parafilm, storing samples and stock solutions in a refrigerator at approximately $4^{\circ} \mathrm{C}$.

We observed no significant difference in NTO or DNAN soil interaction from samples prepared with or without a background solution of 0.005 $\mathrm{mol} \mathrm{L}^{-1} \mathrm{CaCl}_{2}$. All remaining tests used a $0.005 \mathrm{~mol} \mathrm{~L}^{-1} \mathrm{CaCl}_{2}$ background solution. We selected the soil:solution ratio indicating between $10 \%$ and $30 \%$ adsorption and used it in kinetic adsorption and transformation studies. 
Table 7. Soil:solution ratios tested in NTO and DNAN soil adsorption studies.

\begin{tabular}{|l|l|l|}
\hline \multicolumn{1}{|c|}{$\begin{array}{c}\text { Soil:Solution Ratio } \\
\left(\mathrm{g} \mathrm{mL}^{-1}\right)\end{array}$} & \multicolumn{1}{|c|}{$\begin{array}{c}\text { Soil Weight } \\
(\mathrm{g})\end{array}$} & \multicolumn{1}{c|}{$\begin{array}{c}\text { Volume of Solution Containing Solute } \\
(\mathrm{mL})\end{array}$} \\
\hline $1: 4$ & 5 & 20 \\
\hline $1: 10$ & 2 & 20 \\
\hline $1: 20$ & 1 & 20 \\
\hline $1: 40$ & 0.5 & 20 \\
\hline $1: 60$ & 0.33 & 20 \\
\hline
\end{tabular}

The optimal equilibrium soil:solution ratio for NTO in all soils was 1:4 while for DNAN 1:60 ratio worked best for majority of the soils. To observe changes in NTO and DNAN soil adsorption and transformation with time, we conducted a kinetic adsorption study. Here we prepared in triplicate treatment samples and blanks without soil and equilibrated them for 1, 4, 8, 24, 48, 72, 96, and $120 \mathrm{hr}$; we then centrifuged, filtered, and stored them as previously described. We tested aliquots for $\mathrm{pH}$ and $\mathrm{EC}$ and determined the time interval for attaining chemical equilibrium for each soil type. Equilibrium time was defined as the minimum time required to reach a rate of change in solute concentration of less than 5\% in $24 \mathrm{hr}$ (Roy et al. 1992). NTO and DNAN transformation rate constants, $k$, were determined from the change in solute concentration over time, dC/ dt. For first-order rate, the equation takes the following form

$$
-d C / d t=k C \text {. }
$$

The integrated form of the first-order rate law is

$$
\ln C=-k t+\ln C_{0}
$$

where $\mathrm{C}_{0}$ is initial concentration of target analyte in solution. The slope of this plot was used to determine $\mathrm{k}$.

After determining the equilibrium time, we constructed soil adsorption isotherms. Isotherms comprised eight input concentrations: 0.78, 1.56, $3.13,6.25,12.5,25$, 50, and $100 \mathrm{mg} \mathrm{L}^{-1} \mathrm{NTO}$ or $2.03,4.06$, 8.13, 16.25, 32.5, 65, and $130 \mathrm{mg} \mathrm{L}^{-1}$ DNAN in $0.005 \mathrm{~mol} \mathrm{~L}^{-1} \mathrm{CaCl}_{2}$.

We determined adsorption coefficients from measured solution concentrations and calculated sediment concentrations using linear and Freundlich 
adsorption isotherms. The following equation describes the linear isotherm:

$$
S=K_{d} C
$$

where $\mathrm{S}$ is the contaminant concentration in soil $\left(\mathrm{mg} \mathrm{kg}^{-1}\right), \mathrm{K}_{\mathrm{d}}$ is the adsorption coefficient $\left(\mathrm{mL} \mathrm{g}^{-1}\right)$, and $\mathrm{C}$ is the contaminant concentration in solution $\left(\mathrm{mg} \mathrm{L}^{-1}\right)$. The Freundlich isotherm is described by

$$
S=K_{f} C^{n}
$$

where $\mathrm{K}_{\mathrm{f}}$ is the Freundlich adsorption coefficient and $\mathrm{n}$ is an empirical parameter that indicates the affinity of the compound for the adsorbent. We used the regression analysis function in Windows Excel 2010 to determine linear distribution coefficients $\left(\mathrm{K}_{\mathrm{d}}\right)$, confidence intervals for the estimate, $\mathrm{R}^{2}$, and probabilities of parameters being significantly different from zero. The Freundlich equation was used in linearized form to facilitate application of linear regression tools to determine adsorption parameters. Among the soils, we compared parameters from the equation (linear of Freundlich) that best described the data (largest $\mathrm{R}^{2}$ ) for the majority of the soils to elucidate the effect of soils on adsorption. We related determined parameters to measured soil properties, such as their particle size distribution, organic carbon content, and $\mathrm{pH}$, to determine the effect of geochemical heterogeneity on adsorption of IM compounds and to help predict the environmental fate of these compounds in soils.

\subsubsection{NTO transport studies}

To measure the transport of NTO in soils, we conducted saturated column transport studies under steady-state and transient conditions (Dontsova et al. 2006, 2009b). Solutions of NTO were used as the source. We selected some of the soils used in the batch studies to use in column transport experiments. In the column effluent, we determined concentrations of NTO (presence of ATO was monitored but not observed) and a conservative tracer and used these to construct breakthrough curves.

We conducted saturated flow column experiments by using Supelco (Bellefonte, PA) glass tubes ( $7 \mathrm{~cm}$ in length with a $1.18 \mathrm{~cm}$ internal diameter) with PTFE (polytetrafluoroethylene) caps. Small columns minimize the amount of source material needed and also reduce hazardous waste produced in the study. We packed 11- $12 \mathrm{~g}$ of soil homogenously over a bottom 
layer of silanized glass wool that prevented migration of particles. A layer of glass wool overlay the packed soil and acted to evenly distribute incoming flow. To avoid air entrapment, we saturated columns from the bottom up with a $0.005 \mathrm{~mol} \mathrm{~L}^{-1} \mathrm{CaCl}_{2}$ solution. After saturation, we determined pore volume as the volume of solution required to saturate the packed column. We attached Tygon microbore tubing, running from a Cole-Parmer (Vernon Hills, IL) Master flex peristaltic pump, to the top of each column to supply NTO or DNAN solution in $\mathrm{CaBr}_{2}$ background at a given flux. We used a $0.02 \mathrm{~mL} \mathrm{~min}^{-1}$ flow rate or $1.1 \mathrm{~cm} \mathrm{hr}^{-1}$ flux. We continuously collected outflow (effluent) into $4 \mathrm{~mL}$ amber vials by using a Teledyne ISCO (Lincoln, NE) Foxy 200 Fraction collector with a 200 vial capacity. A maximum of ten columns could run simultaneously. After about 6 to 16 pore volumes, depending on the soil, inflow was switched to $0.005 \mathrm{~mol} \mathrm{~L}^{-1}$ $\mathrm{CaCl}_{2}$ solution; and the flow continued for another 6- 16 pore volumes to evaluate NTO desorption from the soil. We analyzed a conservative tracer (bromide, Br) by using Ion Chromatography (Dionex ICS 5000 with diode array) to distinguish between non-equilibrium attributed to physical versus chemical processes and to determine dispersion and diffusion in the columns.

As the same soils were used in batch adsorption and transformation studies, we were able to compare determined parameters, allowing us to verify the processes responsible for NTO attenuation. In addition, as soils from some of these sites have been used in previous explosive and propellants studies (Dontsova et al. 2006, 2007 , 2008, 2009a, 2009b; Taylor et al. 2012), we were able to compare the transport of IM compounds to those of other energetics.

We performed flow-interruption tests, wherein flow was stopped for $24 \mathrm{hr}$, for some experiments to quantify mass- transfer processes, such as diffusion and sorption (Brusseau et al. 1989, 1997). Stopping the flow allows the system to equilibrate. This is reflected in decreases in concentration if degradation or kinetic sorption is taking place.

\subsubsection{IM formulation dissolution and transport studies}

To study dissolution and transport of IMs, we used undetonated particles of IMX101, IM formulation containing DNAN and NTO, and detonation residues as the source (Dontsova et al., 2006, 2009b). Camp Guernsey and Camp Swift soils were used in column transport and IM solid dissolution experiments. For the experiments employing the formulations, we placed 
explosives particles on the surface of soil saturated with $0.005 \mathrm{~mol} \mathrm{~L}^{-1}$ $\mathrm{CaCl}_{2}$ and started the flow of the $0.005 \mathrm{~mol} \mathrm{~L}^{-1} \mathrm{CaBr}_{2}$ solution (with $\mathrm{Br}$ serving as the conservative tracer). Once steady-state outflow was reached, we measured the desorption of explosives from the soil by removing the explosives particles and continuing the flow of the $0.005 \mathrm{M} \mathrm{CaCl}_{2}$ solution until we no longer detected the target analytes in the outflow.

We collected and analyzed outflow from the columns, the water that interacted with the explosives and then with soil, by using a Dionex UHPLC (ultra high performance liquid chromatography) with Diode Array Detector to evaluate dissolution, degradation, and adsorption of IM compounds and their transformation products. The $\mathrm{Br}$ tracer was used to distinguish between physical and chemical non-equilibrium processes and to determine dispersion and diffusion in the columns. We measured concentration of the $\mathrm{Br}$ tracer by using ion chromatography (Dionex ICS 5000 ion chromatograph with conductivity detector, ThermoFisher, CA).

Collecting effluent directly into the vials (Figure 25) prevented contact between the target solute and the plastic tubing, eliminating possible adsorption pathways that can have significant impact on measured solution concentrations.

We determined concentrations of NTO, DNAN, their transformation products, and NQ, which is also present in IMX101, in the column effluent and used these to construct breakthrough curves.

We used a $0.01 \mathrm{~mL} \mathrm{~min}^{-1}$ flow rate $\left(0.55 \mathrm{~cm} \mathrm{hr}^{-1}\right.$ flux $)$ to match drip studies. No flow interruption was done during the IMX101 dissolution experiments. 
Figure 25. Experimental setup for the IM column transport studies.

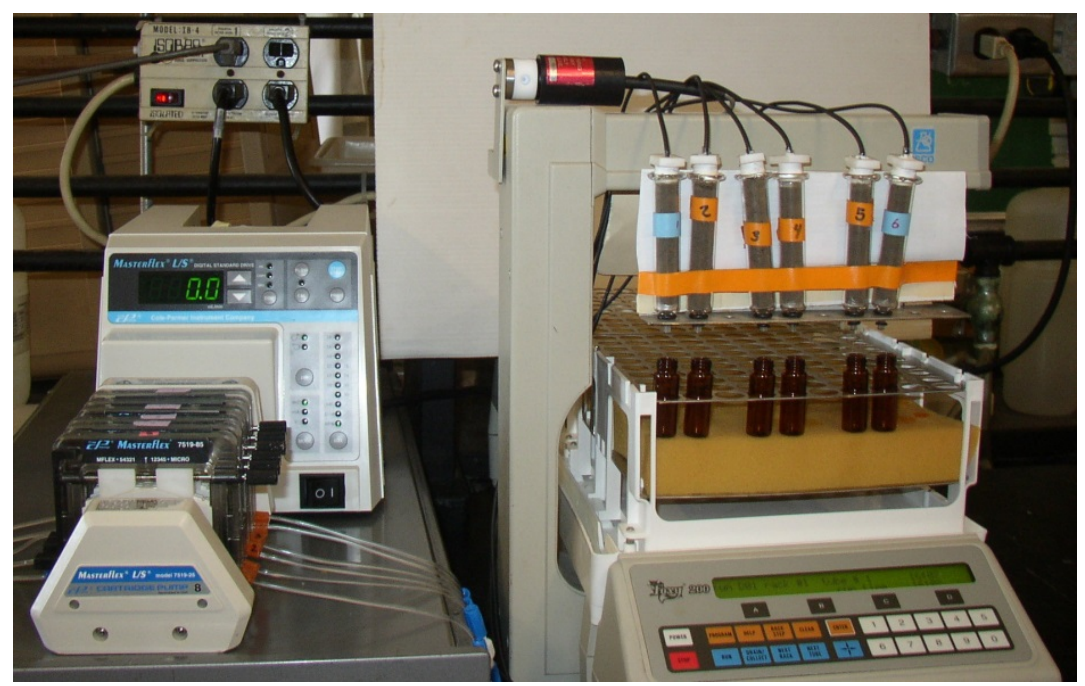

\subsection{Results}

\subsubsection{Soil sample collection and characterization}

We used eleven different soils for this study. Ten of these soils were collected at military training ranges, and Catlin silt loam was collected on the University of Illinois at Urbana-Champaign university farm.

The Catlin and two additional soils, Plymouth sandy loam and Sassafras loam, were previously used to study soil interactions and transport of propellant constituents; studies of high explosives had also used Catlin and Plymouth soils. New soils were sent to us by the Army National GuardEnvironmental Directorate from training sites affiliated with the U.S. Army National Guard (Table 8). Facilities sampled included AZ National Guard Florence Military Reservation; Camp Butner, Army National Guard Training Center, NC; Camp Swift Army National Guard Training Center, TX; Camp Guernsey, a Premier J oint Training Center in Guernsey, WY, serving both a U.S. Air Force and National Guard. Additionally, samples came from two locations in Montana: Fort William Henry Harrison, the Montana National Guard training facility, where small arms, heavy machine gun, and pyrotechnics are fired, and Limestone Hills Training Area, where artillery and grenade training and open burn/ open detonation is done. These soils belong to the same series but contain different amount of organic carbon, twice as much for Fort Harrison compared to Limestone Hills. Samples were also collected on the small arms range at Arnold Air Force Base, Tullahoma, TN. 
Soils that were collected earlier are identified hereafter using their series name while new soils are identified using the location where they were collected. Table 8 lists the soil series name and classification for all soils.

Table 8. Soils used to study interaction of IMs with the soils. Series names and classifications are according to U.S. Taxonomy and location where collected.

\begin{tabular}{|c|c|c|}
\hline Soil name & Soil classification & Location \\
\hline Catlin & $\begin{array}{l}\text { Catlin silt loam, mixed, mesic, superactive } \\
\text { Oxyaquic Argiudoll }\end{array}$ & Urbana, IL \\
\hline Fort Harrison & $\begin{array}{l}\text { Musselshell sandy loam, carbonatic, frigid Aridic } \\
\text { Calciustepts }\end{array}$ & $\begin{array}{l}\text { Fort William Henry } \\
\text { Harrison, MT }\end{array}$ \\
\hline Arnold AFB & $\begin{array}{l}\text { Captina silt loam, siliceous, mesic, typic } \\
\text { Fragiudults }\end{array}$ & $\begin{array}{l}\text { Arnold Air Force Base } \\
\text { (AFB), TN }\end{array}$ \\
\hline Plymouth & $\begin{array}{l}\text { Plymouth loamy sand, mesic, coated Typic } \\
\text { Quartzipsamment }\end{array}$ & $\begin{array}{l}\text { Massachusetts Military } \\
\text { Reservation, MA }\end{array}$ \\
\hline Camp Butner & $\begin{array}{l}\text { Helena sandy loam, mixed, semiactive, thermic } \\
\text { Aquic Hapludults }\end{array}$ & Camp Butner, NC \\
\hline Limestone Hills & $\begin{array}{l}\text { Musselshell sandy loam, carbonatic, frigid Aridic } \\
\text { Calciustepts }\end{array}$ & Limestone Hills, MT \\
\hline Sassafras & Sassafras loam, siliceous, mesic Typic Hapudult & $\begin{array}{l}\text { Aberdeen Proving } \\
\text { Ground, MD }\end{array}$ \\
\hline Camp Gruber & $\begin{array}{l}\text { Verdigris silt loam, mixed, superactive, thermic } \\
\text { Cumulic Hapludolls }\end{array}$ & Camp Gruber, OK \\
\hline Camp Guernsey & $\begin{array}{l}\text { Keeline-Turnercrest loam, mixed, superactive, } \\
\text { calcareous, mesic Ustic Torriorthents }\end{array}$ & Camp Guernsey, WY \\
\hline Florence MR & $\begin{array}{l}\text { Cherioni loam, mixed, superactive, hyperthermic, } \\
\text { shallow Typic Haplodurids }\end{array}$ & $\begin{array}{l}\text { Florence Military } \\
\text { Reservation (MR), AZ }\end{array}$ \\
\hline Camp Swift & $\begin{array}{l}\text { Bergstrom sandy clay loam, mixed, superactive, } \\
\text { thermic Cumulic Haplustolls }\end{array}$ & Camp Swift, TX \\
\hline
\end{tabular}

Selected soils represent a range of climate and conditions on military ranges where live-fire training is occurring (Figure 26). They encompass multiple soil orders, including Entisols, Inceptisols, Mollisols, Aridisols, and Ultisols, with different moisture regimes, from very dry to wet. Table 9 summarizes the physical and chemical properties for the 11 soils selected for this study. All studied soils had mixed particle size, ranging from fine (clay loam) to coarse (loamy sand). 
Figure 26. Map of the continental Unites States indicating location of soil collection.

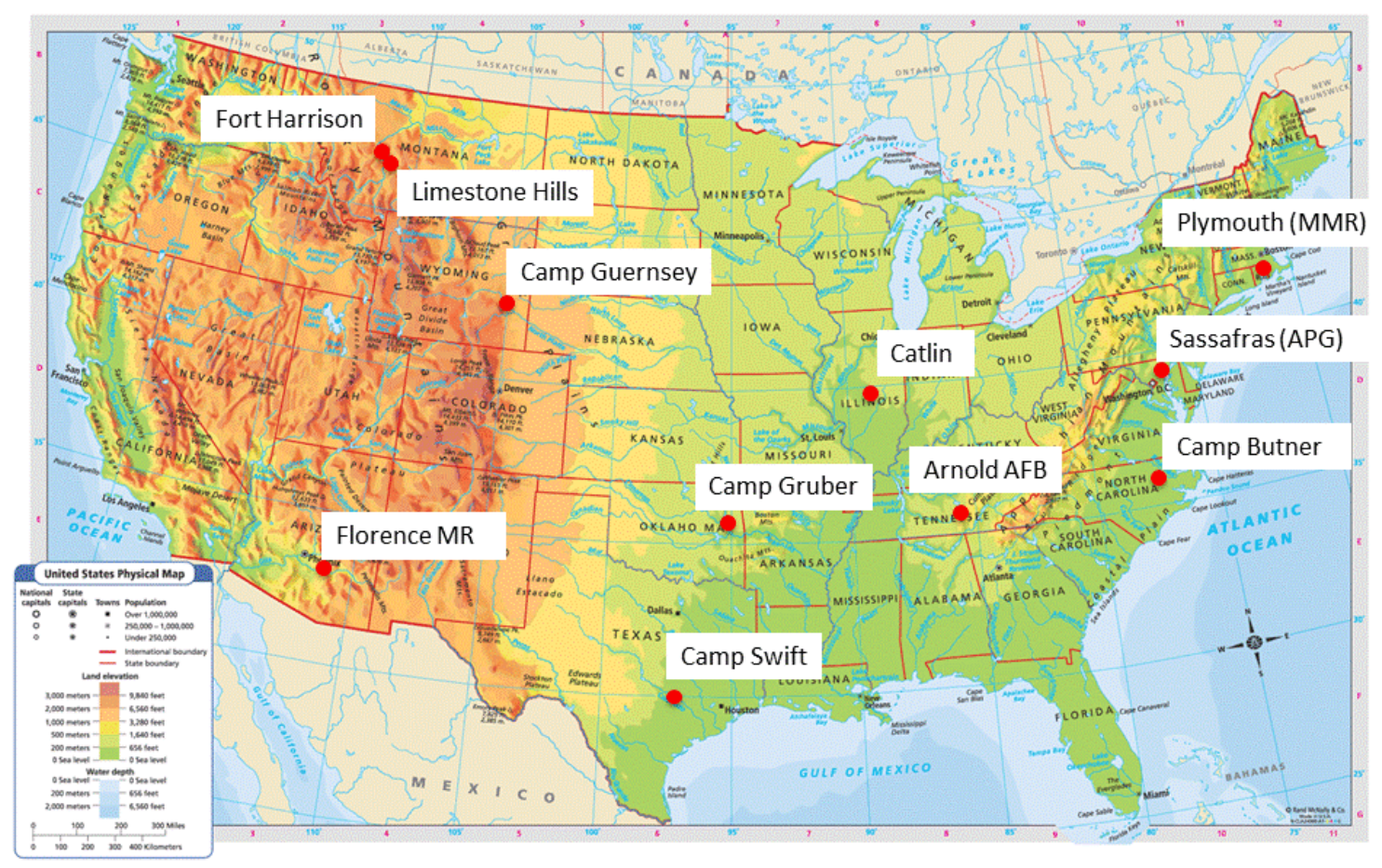

Table 9. Measured physical and chemical properties of soils used in adsorption and transport studies with NTO, DNAN, and IM formulations.

\begin{tabular}{|l|l|r|r|r|r|r|r|r|}
\hline \multicolumn{1}{|c|}{ Soil } & \multicolumn{1}{|c|}{ Texture } & $\begin{array}{c}\text { Clay } \\
(\%)\end{array}$ & \multicolumn{1}{c|}{$\begin{array}{c}\text { Silt } \\
(\%)\end{array}$} & $\begin{array}{c}\text { Sand } \\
(\%)\end{array}$ & \multicolumn{1}{c|}{$\mathrm{pH}^{*}$} & $\begin{array}{c}\mathrm{EC}^{\dagger} \\
\left(\mu \mathrm{cm}^{-1}\right)\end{array}$ & $\begin{array}{c}\text { SSA } \\
\left(\mathrm{m}^{2} \mathbf{g}^{-1}\right)\end{array}$ & $\begin{array}{c}\text { OC\$ } \\
(\%)\end{array}$ \\
\hline Catlin & silt loam & 25.6 & 65.5 & 8.9 & 7.31 & 492 & 6.4 & 5.28 \\
\hline Fort Harrison & sandy loam & 8.7 & 36.5 & 54.9 & 6.67 & 449 & 7.4 & 3.88 \\
\hline Arnold AFB & silt loam & 11.4 & 65.5 & 23.1 & 6.66 & 131 & 7.8 & 2.68 \\
\hline Plymouth & loamy sand & 4.4 & 20.4 & 75.2 & 4.23 & 206 & 1.7 & 2.45 \\
\hline Camp Butner & sandy loam & 7.7 & 25.9 & 66.4 & 6.69 & 219 & 4.8 & 2.42 \\
\hline Limestone Hills & sandy loam & 11.2 & 35.7 & 53.1 & 7.54 & 539 & 10.5 & 1.99 \\
\hline Sassafras & loam & 16.4 & 42.3 & 41.4 & 4.40 & 212 & 7.17 & 1.30 \\
\hline Camp Gruber & clay loam & 32.3 & 44.9 & 22.8 & 5.39 & 74 & 38.3 & 0.83 \\
\hline Camp Guernsey & loam & 4.1 & 12.5 & 83.4 & 8.21 & 477 & 3.9 & 0.77 \\
\hline Florence MR & loam & 26.8 & 33.5 & 39.7 & 8.00 & 417 & 33.0 & 0.45 \\
\hline Camp Swift & sandy clay & 23.7 & 20.8 & 55.6 & 7.83 & 203 & 15.1 & 0.34 \\
& loam & & & & & & & \\
\hline
\end{tabular}

${ }^{*}$ In 1:1 soil:water

$\dagger \mathrm{EC}=$ Electrical conductivity

$\ddagger$ SSA = specific surface area

$\S \mathrm{OC}=$ organic carbon 


\subsubsection{NTO batch adsorption studies}

We designed our preliminary experiments to establish the effect of a background electrolyte, $0.005 \mathrm{~mol} \mathrm{~L}^{-1} \mathrm{CaCl}_{2}$ solution, on sorption of NTO in soils and to determine the soil:solution ratio that would result in $10 \%-$ $30 \%$ retention of NTO on the soil. An adsorption of $10 \%-30 \%$ results in the smallest relative error in adsorption experiments (Roy et al. 1992; McDonald and Evangelou 1997), and a 1:4 soil:solution ratio came closest to the target solute removal for NTO. NTO is negatively charged under the environmental $\mathrm{pH}$ range; and therefore, electrolytes can influence NTO sorption. However, preliminary experiments indicated that there was no effect of background electrolytes on sorption at the concentration used.

Kinetic experiments indicated that the majority of soils reached equilibrium with NTO in solution at $24 \mathrm{hr}$ with the exception of Catlin and Plymouth soils, which reached equilibrium at $8 \mathrm{hr}$, and Limestone Hills soil, which required $48 \mathrm{hr}$. Kinetic experiments also allowed us to determine transformation rate constants for NTO in soils (Figure 27; Table 10). Control NTO solution (blank) did not indicate a decrease in concentration over $120 \mathrm{hr}$ kinetic sorption experiments. When soils were present, the majority of soils had a faster decrease in solution concentrations over the first $24 \mathrm{hr}$ followed by a slower decrease. We detected no organic products of NTO transformation. Figure 27 presents results for the kinetic experiment using Plymouth soil, including a plot of concentrations normalized to input over time and the natural logarithm of this value plotted as a function of time. The slope of the linear regression between time and natural logarithm of the concentration is used to determine the rate of the first-order transformation reaction. Estimates ranged between $0.0004 \mathrm{hr}^{-1}$ in Camp Guernsey and $0.0221 \mathrm{hr}^{-1}$ in Catlin. Rate estimates were highly significant, 99\%, and different from zero for all soils, except for Limestone Hills where it was only significant at the $95 \%$ level.

Measured transformation rates were lower for soils with less OC, such as Camp Guernsey, Florence MR, and Camp Swift. There was a positive relationship between the percent OC present in the soil and the measured transformation rate constant $\mathrm{k}$ (probability, $\mathrm{P}=0.02$ ), however, $\mathrm{R}^{2}$ was low at 0.46 (Figure 28). Other soil parameters, such as clay content, $\mathrm{pH}$, and SSA, poorly correlated with $\mathrm{k}$. The measured NTO transformation rate in Plymouth soil, $0.0043 \mathrm{hr}^{-1}$, was about three times slower than one observed previously for RDX (0.013 hr-1) (Dontsova et al. 2006). 
Figure 27. Plot of (a) NTO solution concentrations normalized by input over time in the presence of Plymouth soil and (b) the same concentrations plotted as the natural logarithm of concentrations in order to determine the transformation rate constant of NTO. Error bars equal one standard error of the mean ( $n=9$ with 3 independent replicate test tubes and 3 repeat HPLC measurements for each sample).
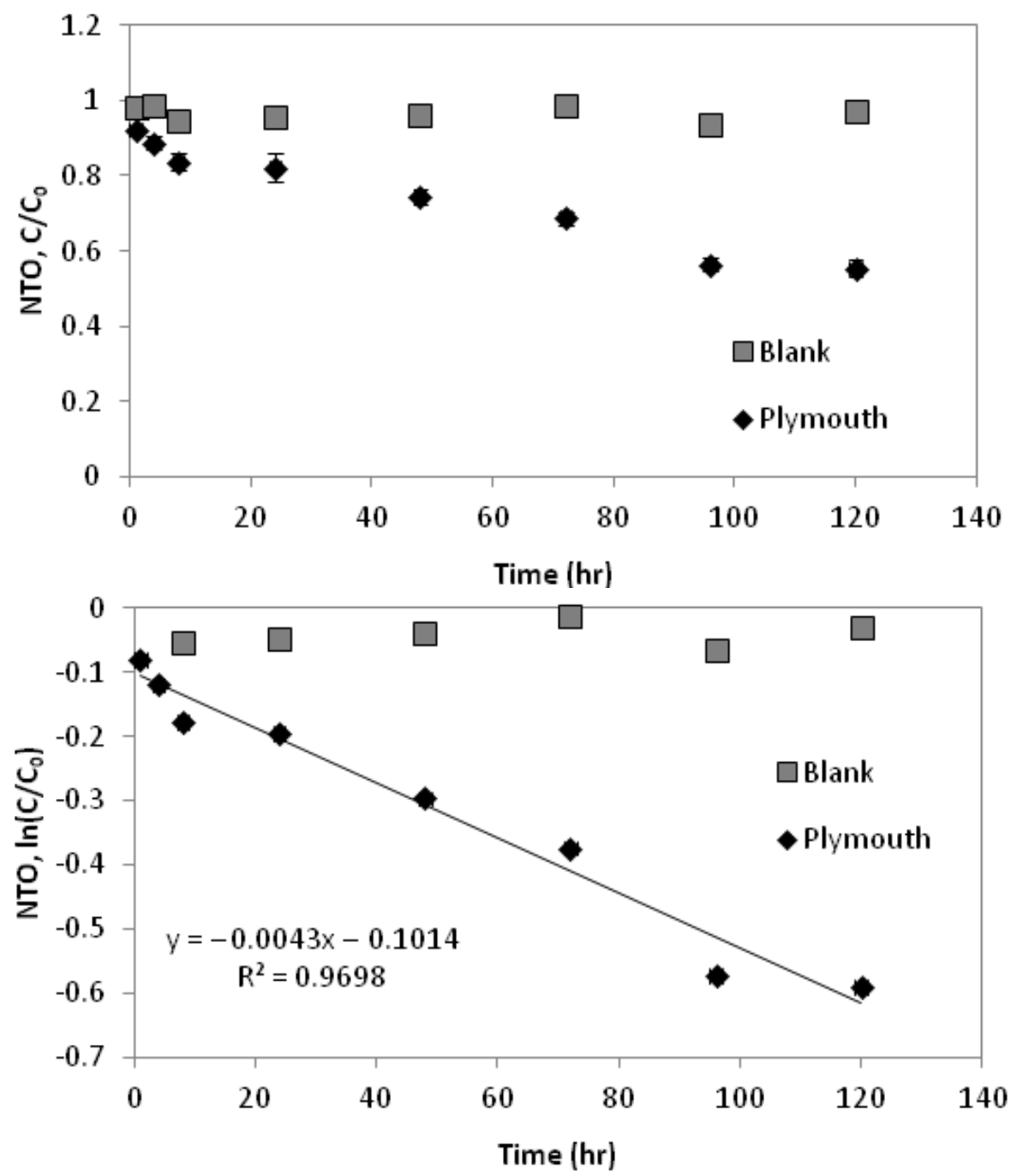

NTO adsorbed very weakly to the studied soils as can be expected for a negatively charged compound in a matrix that also possesses a net negative charge. All determined adsorption coefficient $\left(K_{d}\right)$ values were less than $1 \mathrm{~cm}^{3} \mathrm{~g}^{-1}$ with the largest observed $K_{d}$ equal to $0.51 \mathrm{~cm}^{3} \mathrm{~g}^{-1}$ in Camp Gruber soil. For most soils, both Freundlich and linear adsorption isotherm described the observed adsorption well; but Freundlich usually resulted in a slightly better fit (Figures 29-32; Table 10). The Freundlich parameter $\mathrm{n}$ was not similar among the soils, with the majority smaller than one, but was above one for the two soils with the highest OC content, Catlin and Fort Harrison. Fits to both linear and Freundlich isotherms were 
worse for soils that had very little adsorption (Camp Guernsey, Florence $\mathrm{MR}$, and Camp Swift had $\mathrm{K}_{\mathrm{d}}$ less than $0.1 \mathrm{~cm}^{3} \mathrm{~g}^{-1}$ and Camp Butner 0.12 $\mathrm{cm}^{3} \mathrm{~g}^{-1}$ ) due to a larger percentage of error for these measurements.

Table 10. Fate and transport parameters for NTO in studied soils: Freundlich adsorption parameters, $K_{f}$ and $n$, soil adsorption coefficient, $K_{d}$, adsorption coefficient normalized to fraction of organic carbon in soils, Koc; transformation rate constant, $k$, and $R^{2}$ values for the fits.

\begin{tabular}{|c|c|c|c|c|c|c|c|c|}
\hline Soil & $K_{f}$ & $n$ & $R^{2}$ & $\begin{array}{c}K_{d} \\
\left(\mathrm{~cm}^{3}\right. \\
\left.\mathrm{g}^{-1}\right)\end{array}$ & $R^{2}$ & $\begin{array}{c}K_{o c} \\
\left(\mathrm{~cm}^{3} \mathrm{~g}^{-1}\right)\end{array}$ & $\begin{array}{c}k \\
\left(\mathrm{~h}^{-1}\right)\end{array}$ & $R_{2}$ \\
\hline Catlin & 0.21 & 1.03 & 0.94 & 0.21 & 0.92 & 3.98 & 0.0221 & 0.84 \\
\hline Fort Harrison & 0.27 & 1.07 & 0.98 & 0.35 & 0.95 & 9.02 & 0.0021 & 0.98 \\
\hline Arnold AFB & 0.58 & 0.86 & 0.98 & 0.34 & 0.94 & 12.69 & 0.0044 & 0.94 \\
\hline Plymouth & 0.82 & 0.89 & 0.99 & 0.50 & 0.96 & 20.41 & 0.0043 & 0.97 \\
\hline Camp Butner & 0.74 & 0.54 & 0.77 & 0.12 & 0.72 & 4.96 & 0.0021 & 0.98 \\
\hline Limestone Hills & 0.33 & 0.88 & 0.92 & 0.21 & 0.92 & 10.55 & 0.0123 & 0.60 \\
\hline Sassafras & 0.90 & 0.86 & 0.99 & 0.48 & 0.96 & 36.92 & 0.0080 & 0.99 \\
\hline Camp Gruber & 0.54 & 0.99 & 1.00 & 0.51 & 0.99 & 61.45 & 0.0025 & 0.98 \\
\hline Camp Guernsey & 0.06 & 0.48 & 0.18 & 0.02 & 0.21 & 2.60 & 0.0004 & 0.75 \\
\hline Florence MR & 0.09 & 0.77 & 0.55 & 0.06 & 0.57 & 13.33 & 0.0005 & 0.91 \\
\hline Camp Swift & 0.10 & 0.84 & 0.92 & 0.04 & 0.59 & 11.76 & 0.0009 & 0.93 \\
\hline
\end{tabular}

Figure 28. Correlation between measured NTO transformation rate constants $(k)$ and soil OC. $\mathrm{P}=0.02250$.

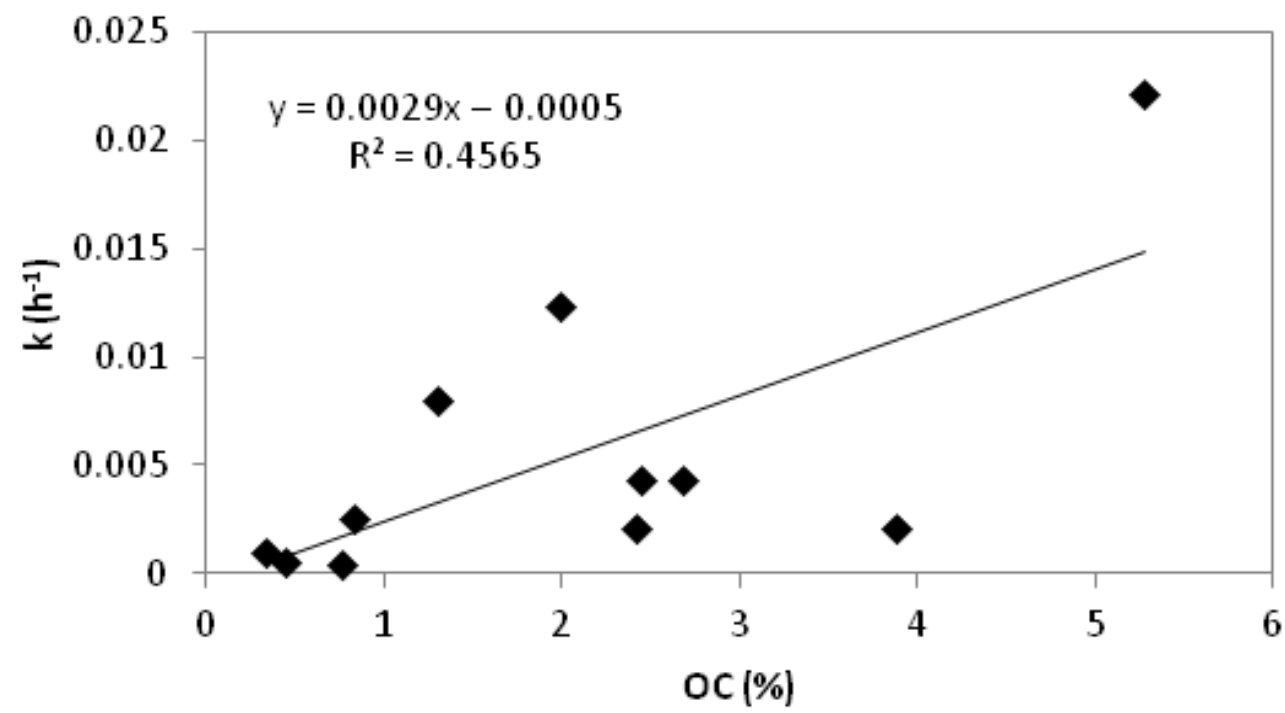


Figure 29. Adsorption isotherms calculated for NTO in Catlin, Fort Harrison, and Limestone Hills soils. The red dashed line indicates the linear adsorption isotherm fit to the measured adsorption data (equation is in red) while the solid black line is a fit of the Freundlich isotherm with the equation presented in black.
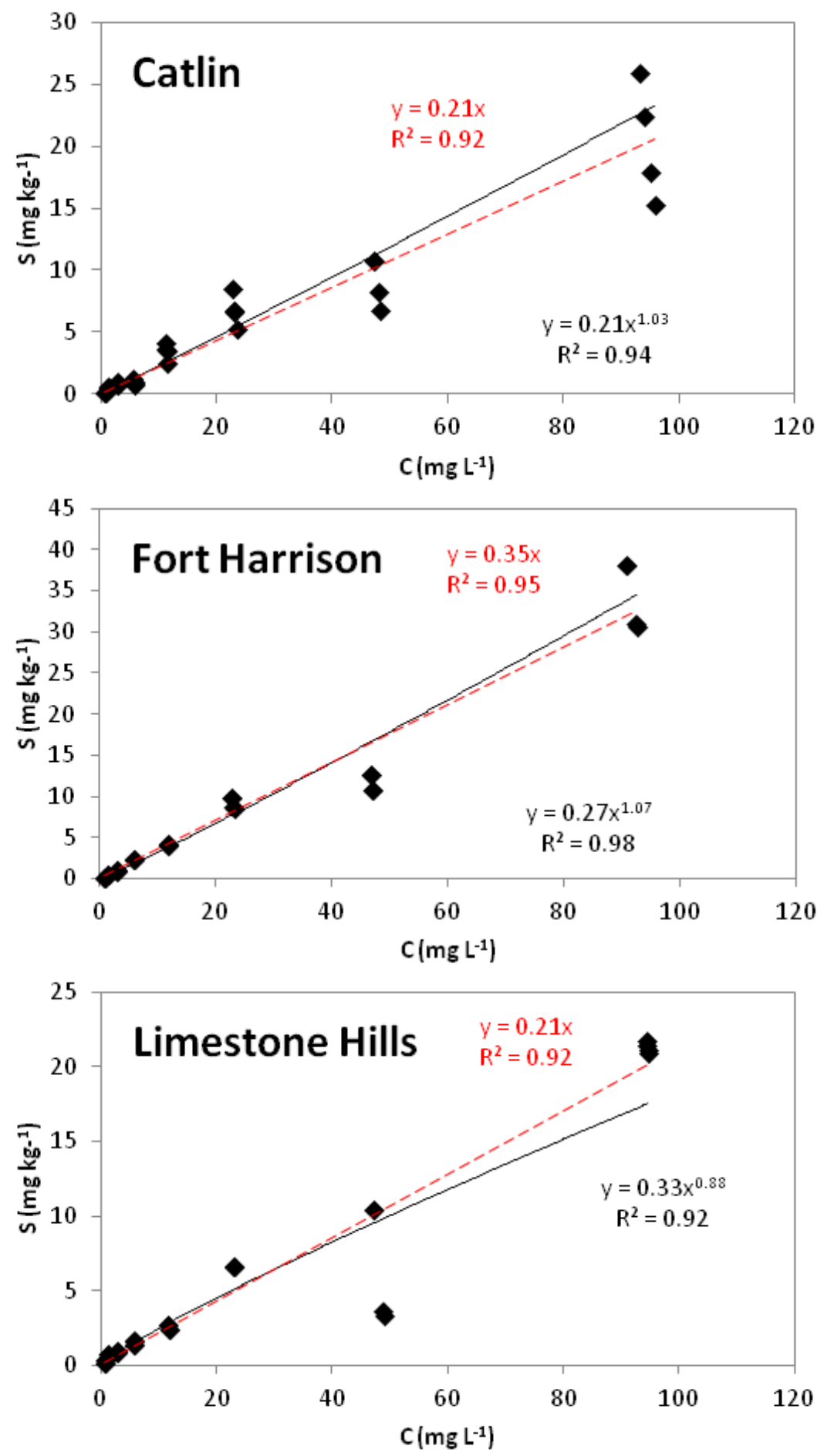
Figure 30. Adsorption isotherms calculated for NTO in Sassafras, Plymouth, and Camp Gruber soils. The red dashed line indicates the linear adsorption isotherm fit to the measured adsorption data (equation is in red) while the solid black line is a fit of the Freundlich isotherm with the equation presented in black.
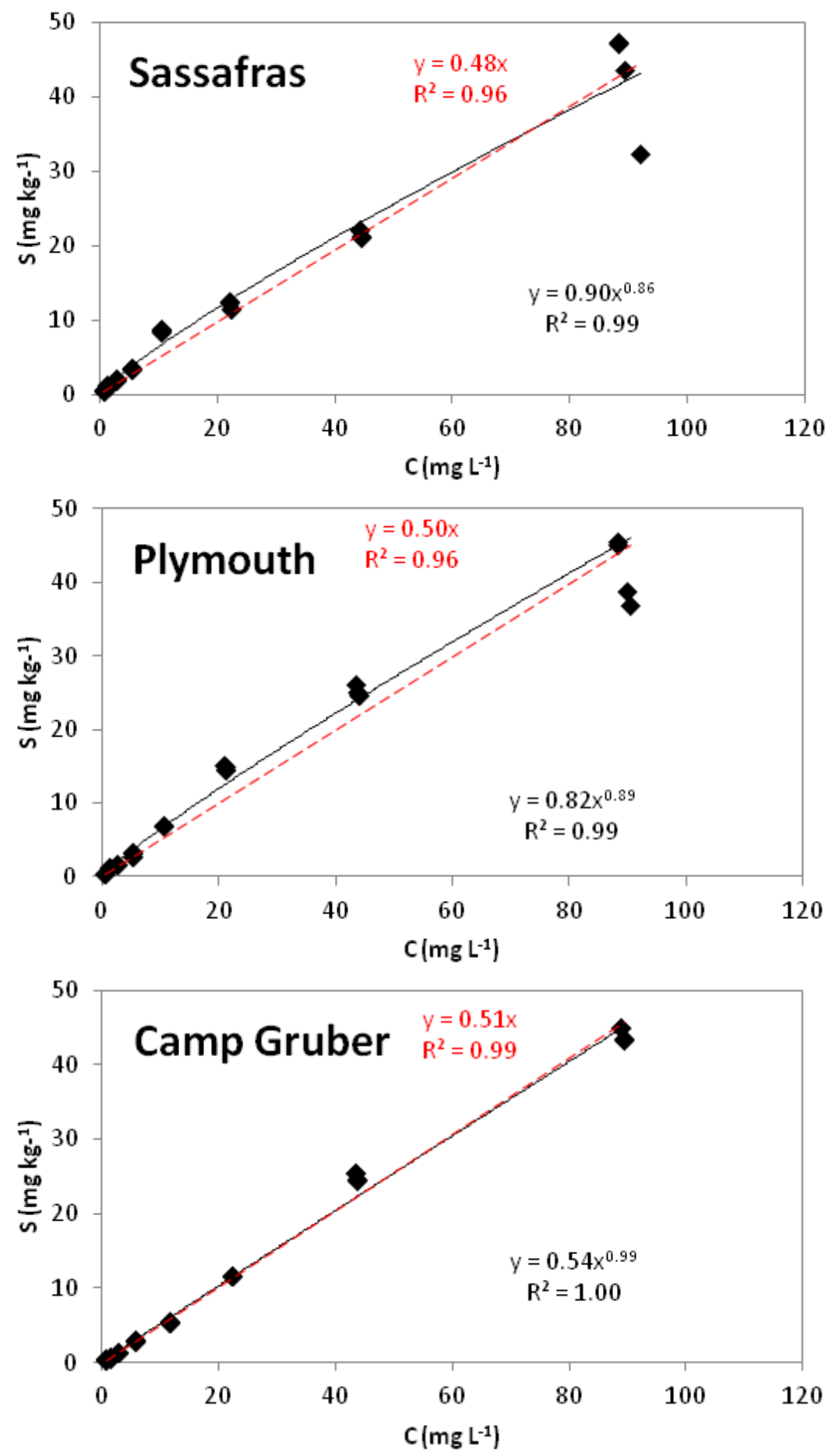
Figure 31. Adsorption isotherms calculated for NTO in Camp Butner, Florence MR, and Camp Guernsey soils. The red dashed line indicates the linear adsorption isotherm fit to the measured adsorption data (equation is in red) while the solid black line is a fit of the Freundlich isotherm with the equation presented in black.
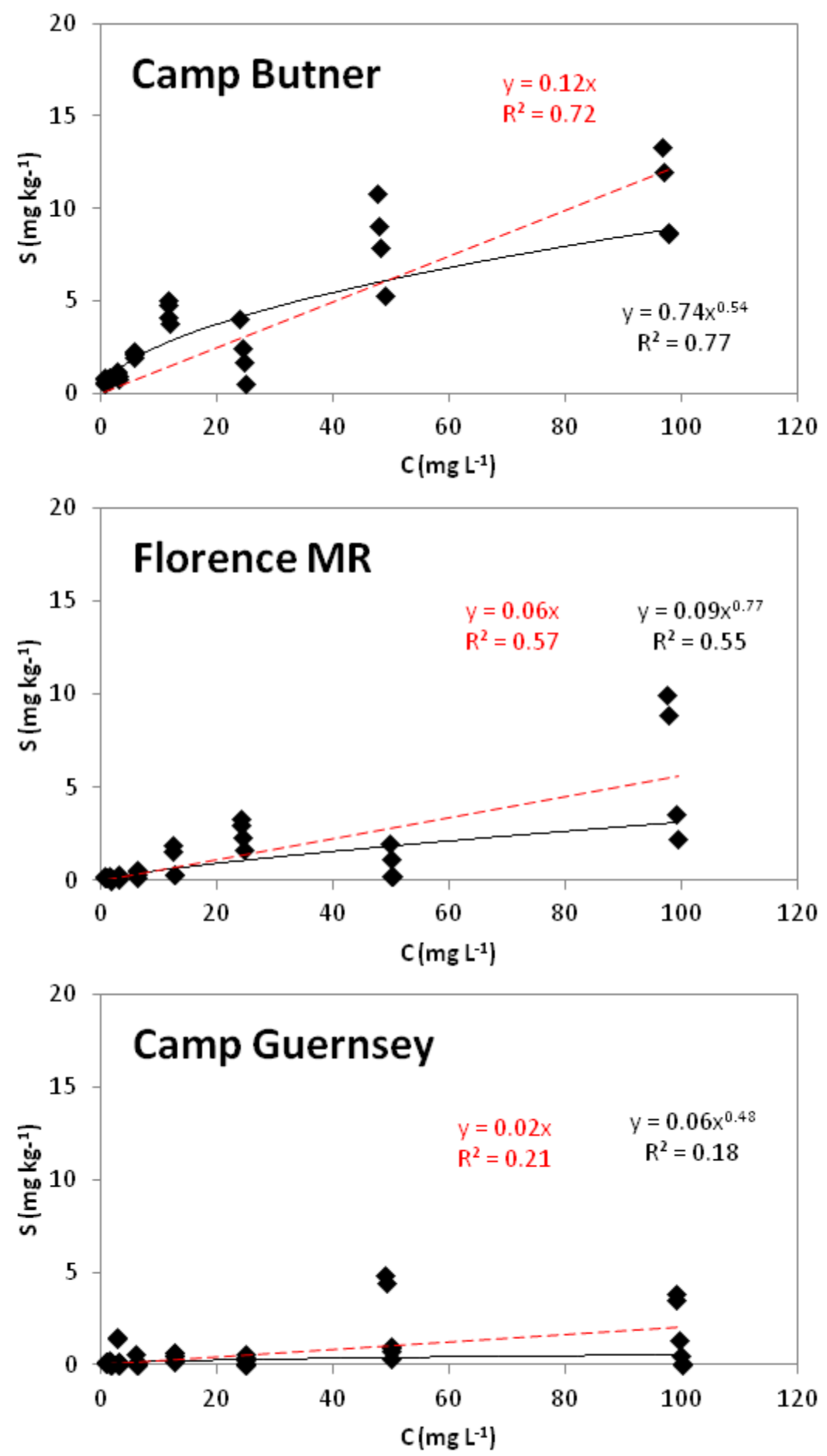
Figure 32. Adsorption isotherms calculated for NTO in Arnold AFB and Camp Swift soils. The red dashed line indicates the linear adsorption isotherm fit to the measured adsorption data (equation is in red) while the solid black line is a fit of the Freundlich isotherm with the equation presented in black.
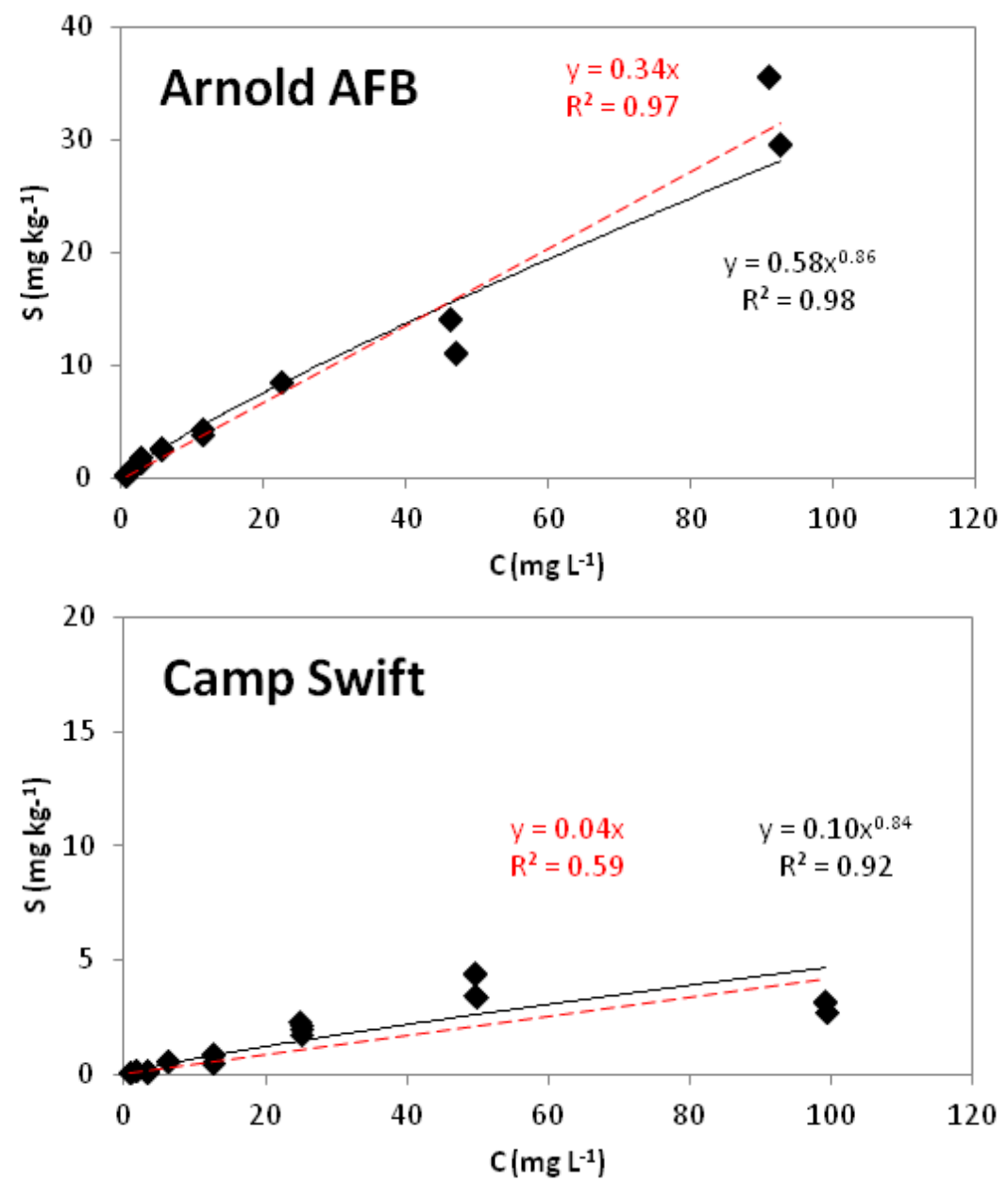

Soil $\mathrm{pH}$ was the strongest indicator of NTO affinity for soil surfaces (Figure 33). There was a highly significant negative relationship between linear adsorption coefficients and soil $\mathrm{pH}$. We observed no relationship between $\mathrm{K}_{\mathrm{d}}$ values and $\mathrm{OM}$, clay, or SSA. The $\mathrm{K}_{\mathrm{f}}$ values had similar if slightly weaker $\left(\mathrm{R}^{2}=0.7818, \mathrm{P}=0.00030\right)$ negative linear relationships with $\mathrm{pH}$. 
Figure 33. Measured NTO adsorption coefficients $\left(K_{d}\right)$ plotted against soil $\mathrm{pH} . \mathrm{P}=0.00011$.

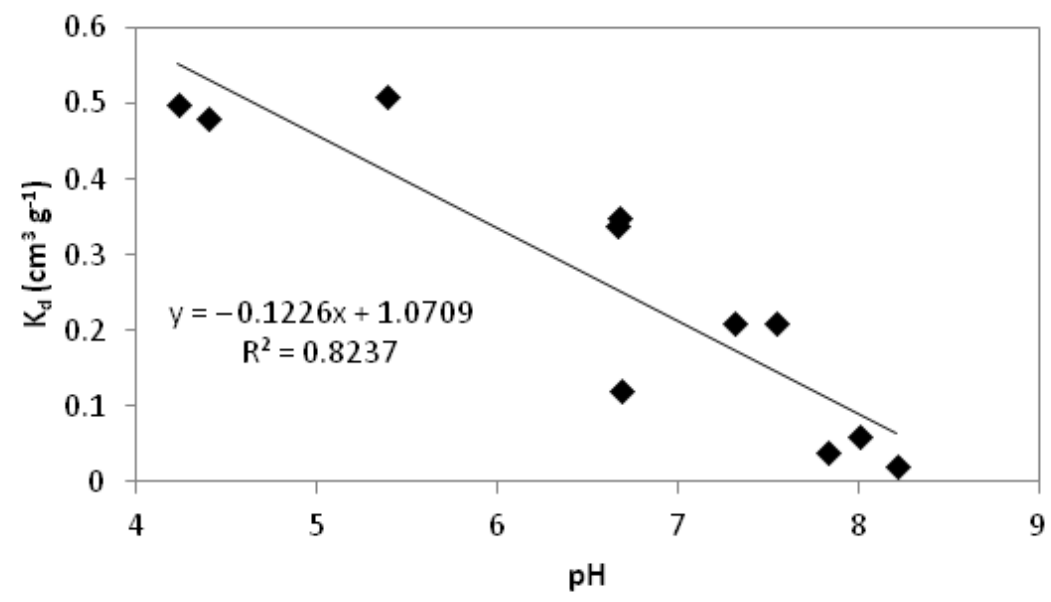

For Catlin soil that has a high $\mathrm{pH}$ and a high OC content, NTO was absorbed about 10 times less than RDX, 0.21 versus $2.03 \mathrm{~cm}^{3} \mathrm{~g}^{-1}$ (Dontsova et al. 2009a), while for Plymouth soil (low pH, less OC) NTO was more strongly adsorbed than RDX, 0.82 versus $0.65 \mathrm{~cm}^{3} \mathrm{~g}^{-1}$ (Dontsova et al. 2006).

\subsubsection{DNAN batch adsorption studies}

We found that the best soil:solution ratio for the majority of DNAN adsorption experiments was 1:60. We observed no effect of the background electrolyte solution ( $0.005 \mathrm{~mol} \mathrm{~L}^{-1} \mathrm{CaCl}_{2}$ ) on adsorption. Kinetic adsorption studies conducted to determine the best equilibration time for obtaining isotherms and to determine the transformation rate of DNAN in soils indicated that the concentration of DNAN in the control (blank-no soil) samples did not change over $120 \mathrm{hr}$ (Figure 34). For samples with soil, however, there was a sharp drop in DNAN concentrations in the first hours followed by a steady decrease in concentration. This change followed first-order kinetics.

Transformation rate constants, $\mathrm{k}$, ranged between 0.0006 and $0.0073 \mathrm{hr}^{-1}$ for Camp Swift and Camp Gruber soils, resectively. Although $\mathrm{k}$ appears to increase with $\mathrm{OC}$, the trend is not statistically significant $(\mathrm{P}=0.78101)$. Our study found that other soil parameters, such as $\mathrm{pH}$, clay content, and SSA, are not correlated with $\mathrm{k}$. No known products of DNAN tranformation were measured in the solutions. All soils reached equilibrium at $24 \mathrm{hr}$, except Sassafras loam, which reached equilibrium with DNAN at $48 \mathrm{hr}$. 
Figure 34. Plot of (a) normalized DNAN solution concentrations in contact with Camp Guernsey soil and (b) the same concentrations plotted as the natural logarithm whose slope gives the transformation rate, $k$, of NTO.
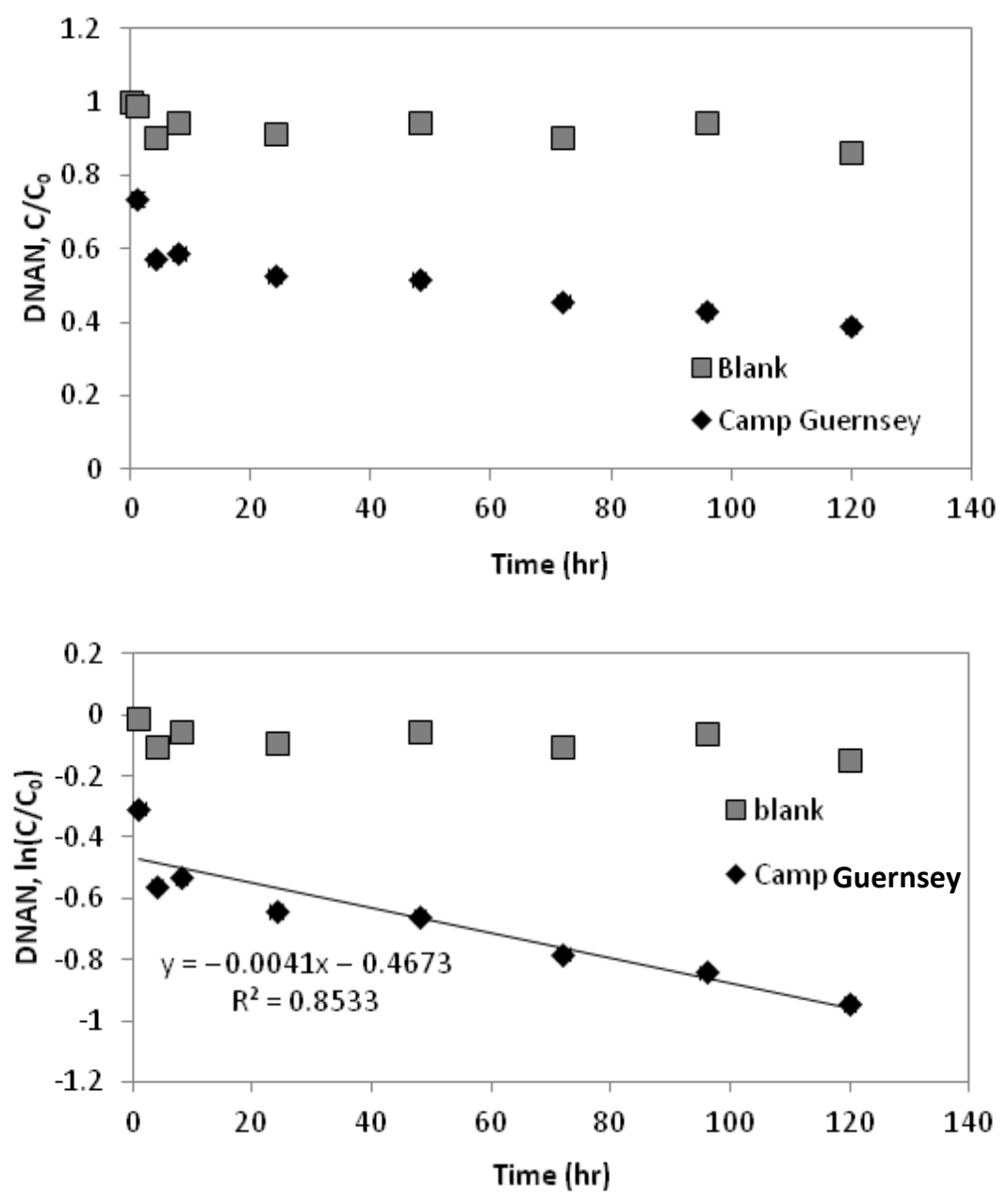

DNAN transformation was significantly slower than observed for TNT. For Plymouth soil, the TNT transformation rate constant was $0.21 \mathrm{hr}^{-1}$ (Dontsova et al. 2006) while the DNAN constant was $0.0070 \mathrm{hr}^{-1}$.

For all studied soils, the Freundlich isotherm resulted in a highly significant fit to the data. Isotherms were similar in shape (Figures 35- 38). Parameter $\mathrm{n}$, an exponent in the Freundlich equation, was close for different soils, estimates averaging 0.76 with a range between 0.56 and 0.97 . However, $95 \%$ confidence intervals of $n$ estimates overlapped for the majority of the soils, indicating that they were not significantly different from each other at 95\%. For three soils, Sassafras, Camp Guernsey and Camp Swift, $n$ was not significantly different from 1 (resulting in a linear isotherm). 
Figure 35. Adsorption isotherms calculated for DNAN in Arnold AFB, Camp

Swift, and Sassafras soils. The red dashed line indicates the linear adsorption isotherm fit to the measured adsorption data (equation is in red)

while the solid black line is a fit of the Freundlich isotherm with the equation presented in black.
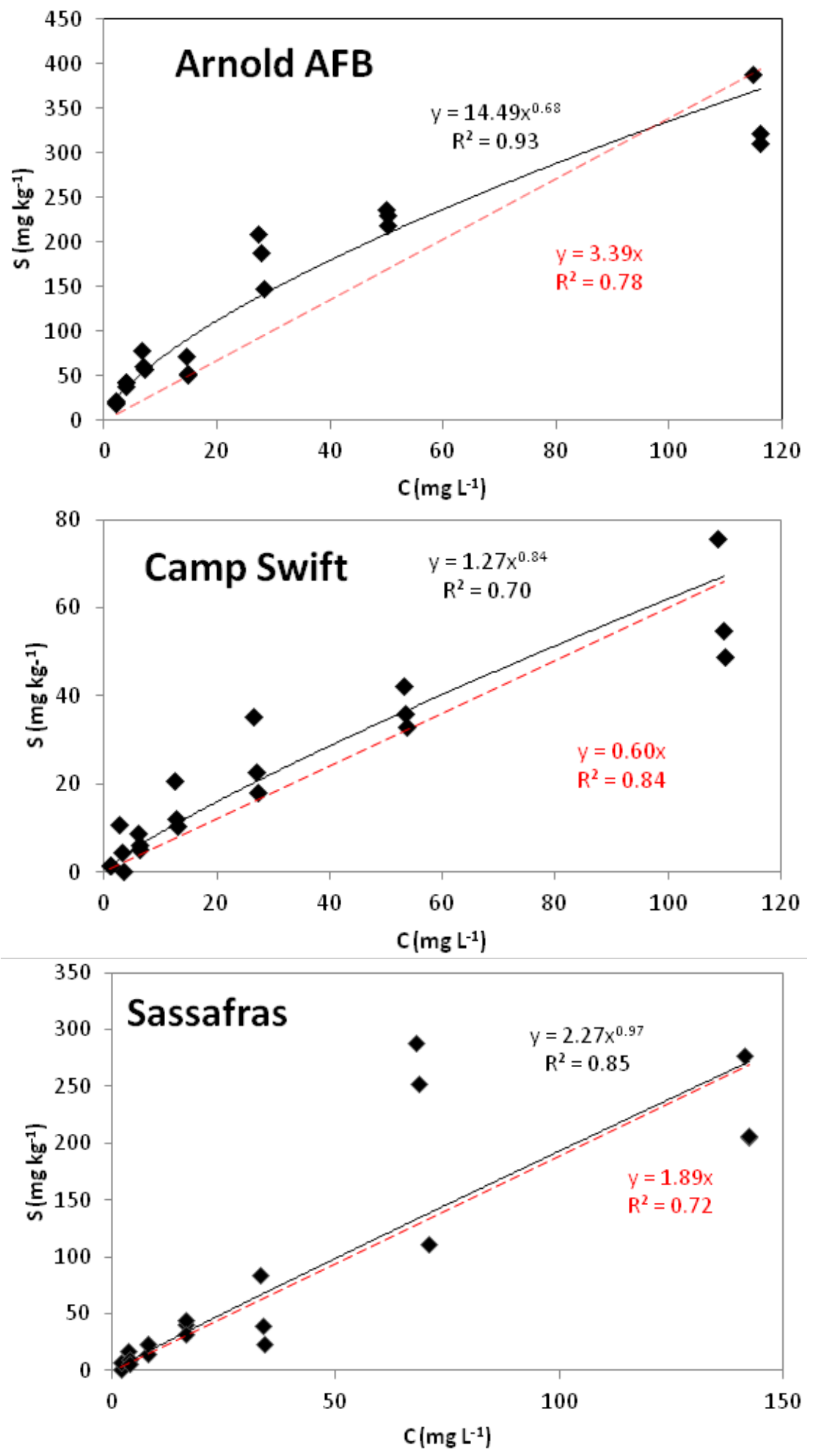
Figure 36. Adsorption isotherms calculated for DNAN in Catlin, Florence

MR, and Camp Gruber soils. The red dashed line indicates the linear adsorption isotherm fit to the measured adsorption data (equation is in red) while the solid black line is a fit of the Freundlich isotherm with the equation presented in black.
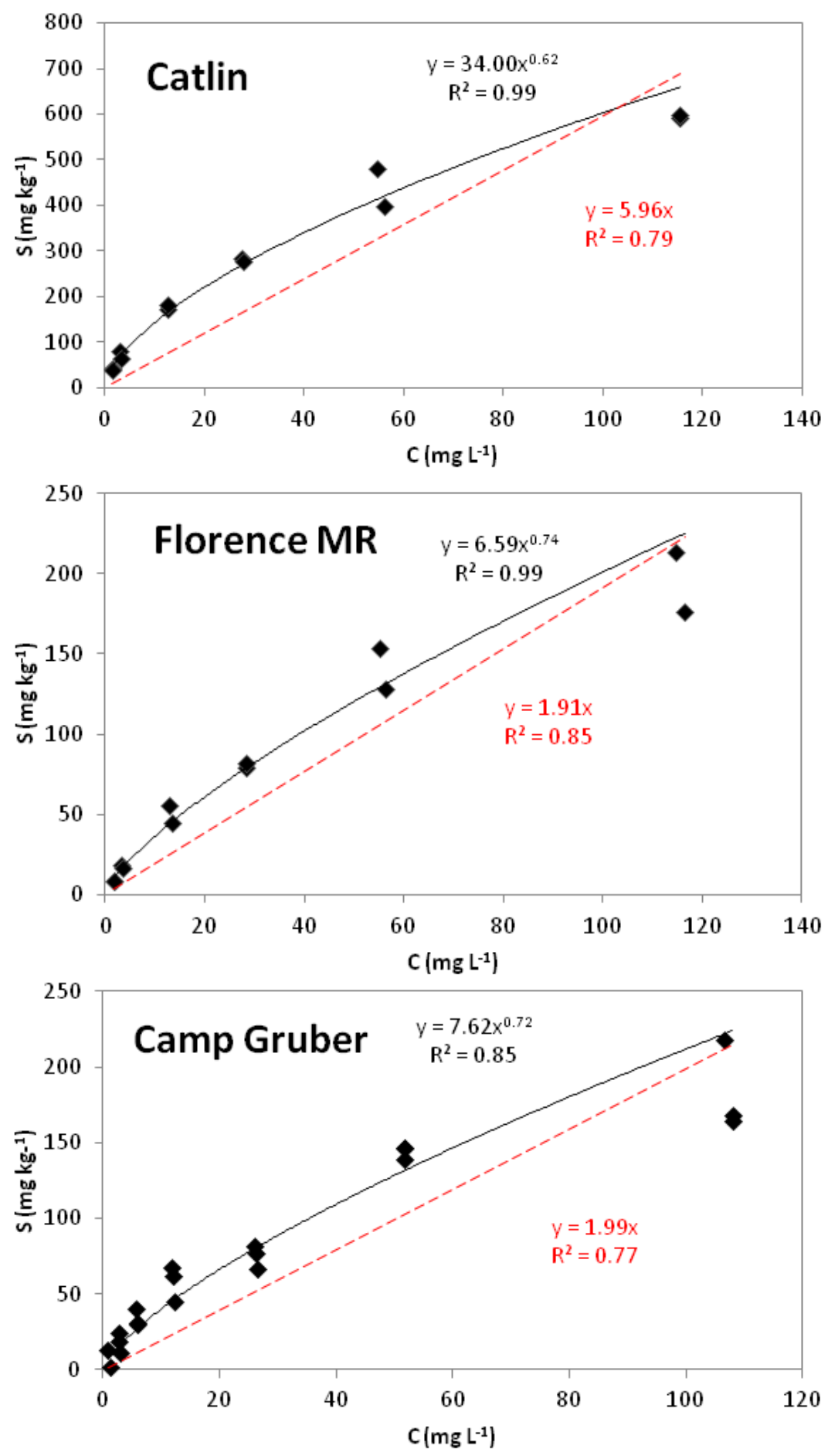
Figure 37. Adsorption isotherms calculated for DNAN in Camp Guernsey, Camp Butner, and Plymouth soils. The red dashed line indicates the linear adsorption isotherm fit to the measured adsorption data (equation is in red) while the solid black line is a fit of the Freundlich isotherm with the equation presented in black.
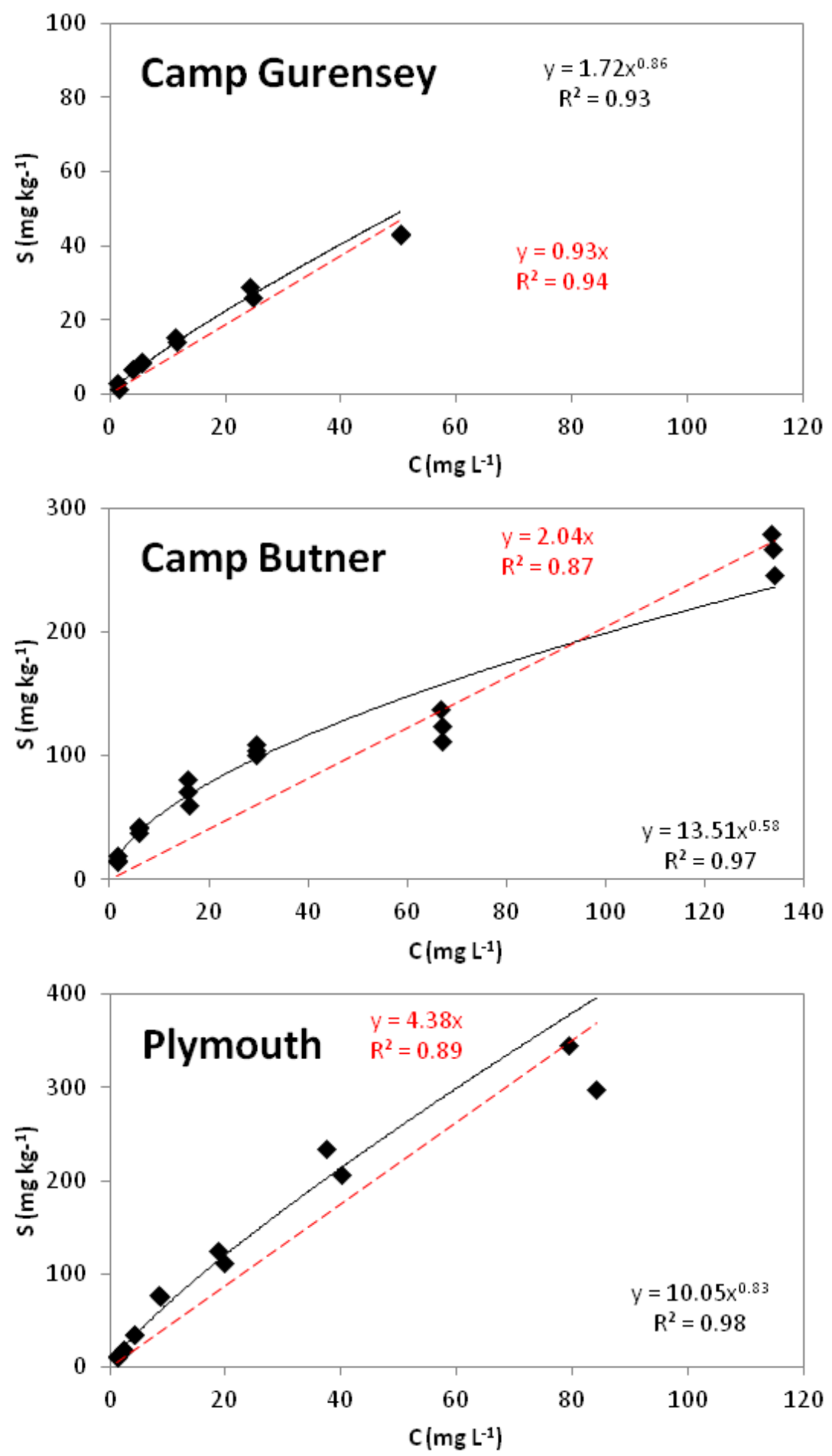
Figure 38. Adsorption isotherms calculated for DNAN in Limestone Hills and Fort Harrison soils. The red dashed line indicates the linear adsorption isotherm fit to the measured adsorption data (equation is in red) while the solid black line is a fit of the Freundlich isotherm with the equation presented in black.
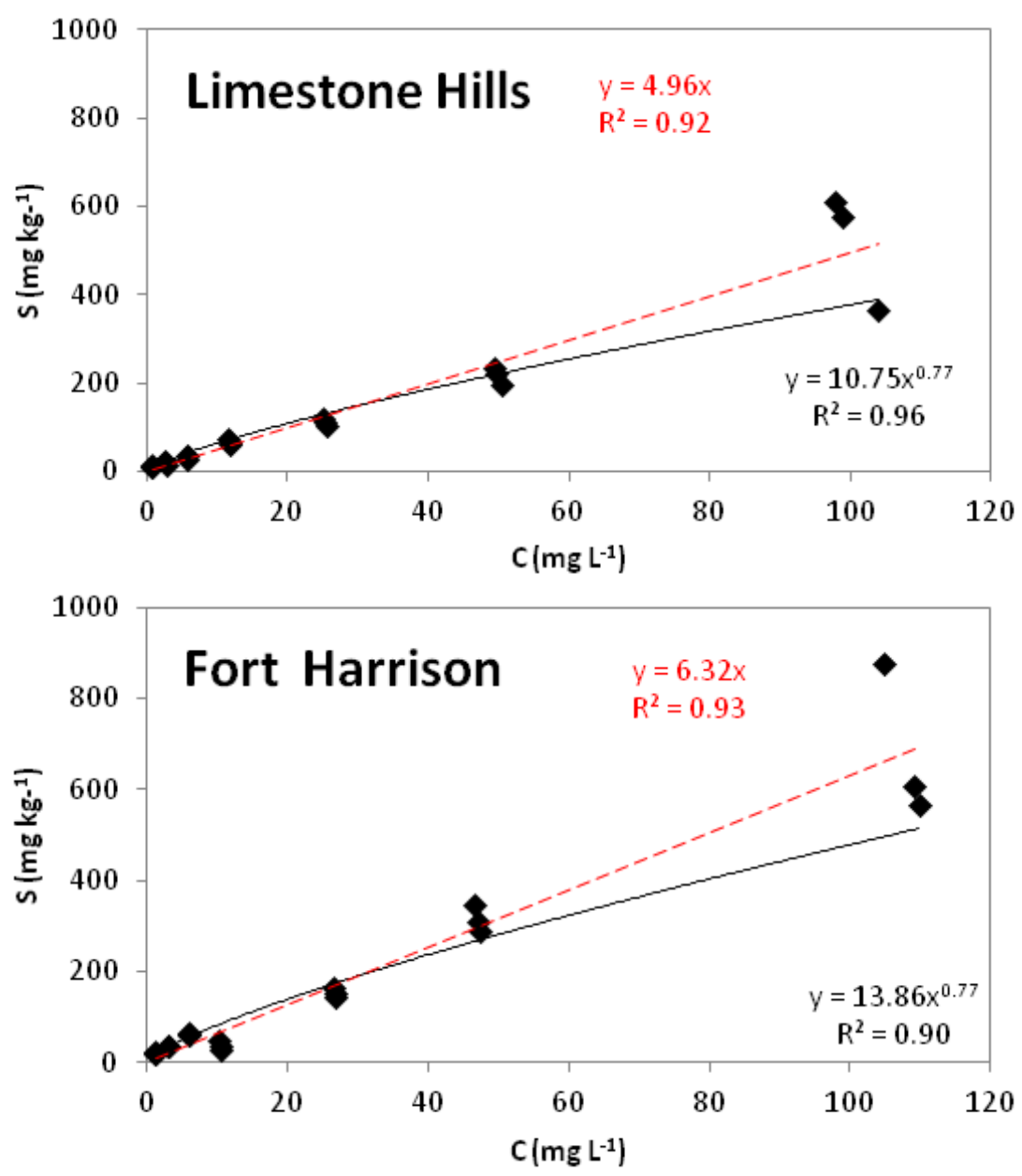

Linear regressions were also highly significant with slopes ( $K_{d}$ values) significantly different from zero. While, for the majority of soils, a Freundlich isotherm described DNAN adsorption the best, for four soils, Fort Harrison, Sassafras, Camp Guernsey, and Camp Swift, a linear isotherm resulted in a better fit to the data (Table 11). Normalizing estimated $K_{d}$ values to OC content in the soil $\left(\mathrm{K}_{\mathrm{OC}}\right)$ resulted in a decrease in the percent standard deviation of the estimates (from $61 \%$ to $49 \%$ ). 
Table 11. Fate and transport parameters for DNAN in studies soils: Freundlich adsorption parameters, $K_{f}$ and $n$, linear adsorption coefficient, $K_{d}$, adsorption coefficient normalized to fraction of organic carbon in soils, $K o c$; transformation rate constant, $k$, and $R^{2}$ values for the fits.

\begin{tabular}{|l|r|r|r|r|r|r|r|r|}
\hline \multicolumn{1}{|c|}{ Soils } & \multicolumn{1}{c|}{$K_{f}$} & \multicolumn{1}{c|}{$n$} & \multicolumn{1}{c|}{$R^{2}$} & $\begin{array}{r}K_{d} \\
\left(\mathrm{~cm}^{3} \mathrm{~g}^{-1}\right)\end{array}$ & \multicolumn{1}{c|}{$R^{2}$} & $\begin{array}{c}K_{o c} \\
\left(\mathrm{~cm}^{3} \mathrm{~g}^{-1}\right)\end{array}$ & \begin{tabular}{c}
\multicolumn{1}{c|}{$\boldsymbol{K}$} \\
$\left(\mathrm{h}^{-1}\right)$
\end{tabular} & \multicolumn{1}{c|}{$\boldsymbol{R}^{\mathbf{2}}$} \\
\hline Catlin & 34.00 & 0.62 & 0.98 & 5.95 & 0.92 & 112.7 & 0.0047 & 0.88 \\
\hline Fort Harrison & 13.86 & 0.77 & 0.9 & 6.32 & 0.93 & 162.9 & 0.0010 & 0.45 \\
\hline Arnold AFB & 14.4 & 0.68 & 0.93 & 3.39 & 0.78 & 126.5 & 0.0022 & 0.92 \\
\hline Plymouth & 10.05 & 0.83 & 0.98 & 4.38 & 0.94 & 178.6 & 0.0070 & 0.51 \\
\hline Camp Butner & 15.35 & 0.56 & 0.91 & 2.05 & 0.92 & 84.8 & 0.0018 & 0.67 \\
\hline Limestone Hills & 10.75 & 0.77 & 0.96 & 4.96 & 0.92 & 250 & 0.0019 & 0.78 \\
\hline Sassafras & 2.26 & 0.97 & 0.85 & 1.89 & 0.72 & 228.3 & 0.0013 & 0.54 \\
\hline Camp Gruber & 7.62 & 0.72 & 0.84 & 1.99 & 0.91 & 240.6 & 0.0073 & 0.29 \\
\hline Camp Guernsey & 1.72 & 0.85 & 0.93 & 0.93 & 0.97 & 121.4 & 0.0041 & 0.85 \\
\hline Florence MR & 6.59 & 0.74 & 0.98 & 1.91 & 0.94 & 424.4 & 0.0007 & 0.35 \\
\hline Camp Swift & 1.27 & 0.84 & 0.7 & 0.6 & 0.84 & 175.5 & 0.0006 & 0.96 \\
\hline
\end{tabular}

Determined DNAN adsorption coefficients, both $\mathrm{K}_{\mathrm{d}}$ and $\mathrm{K}_{\mathrm{f}}$, resulted in highly significant positive relationships with percent OC in the soil (Figure 39). No other measured soil properties, such as clay content, pH, or SSA, correlated with $\mathrm{K}_{\mathrm{d}}$ or $\mathrm{K}_{\mathrm{f}}$ values.

Comparing DNAN with TNT adsorption coefficients for Catlin and Plymouth soils showed higher adsorption of DNAN in Plymouth soil $\left(\mathrm{K}_{\mathrm{d}}\right.$ of 4.38 $\mathrm{cm}^{3} \mathrm{~g}^{-1}$ for DNAN and 0.63 to $1.6 \mathrm{~cm}^{3} \mathrm{~g}^{-1}$ for TNT, depending on the method used) (Dontsova et al. 2006) but less adsorption of DNAN in Catlin soil. Although the Freundlich parameters were similar for two compounds in the Catlin soil $\left(\mathrm{K}_{\mathrm{f}}=34.00, \mathrm{n}=0.62\right.$ for DNAN and $\mathrm{K}_{\mathrm{f}}=32.67, \mathrm{n}$ $=0.60$ for TNT), the $K_{d}$ values were higher for TNT (17.9 vs. 5.95) (Dontsova et al. 2009a). 
Figure 39. Correlation between (a) measured DNAN linear $\left(K_{d}\right)$ and $(b)$ Freundlich $(K)$ adsorption coefficients and soil percent OC. $\mathrm{P}=0.00061$ for $K_{d}$ and 0.00011 for $K_{f}$, both highly significant relationships.
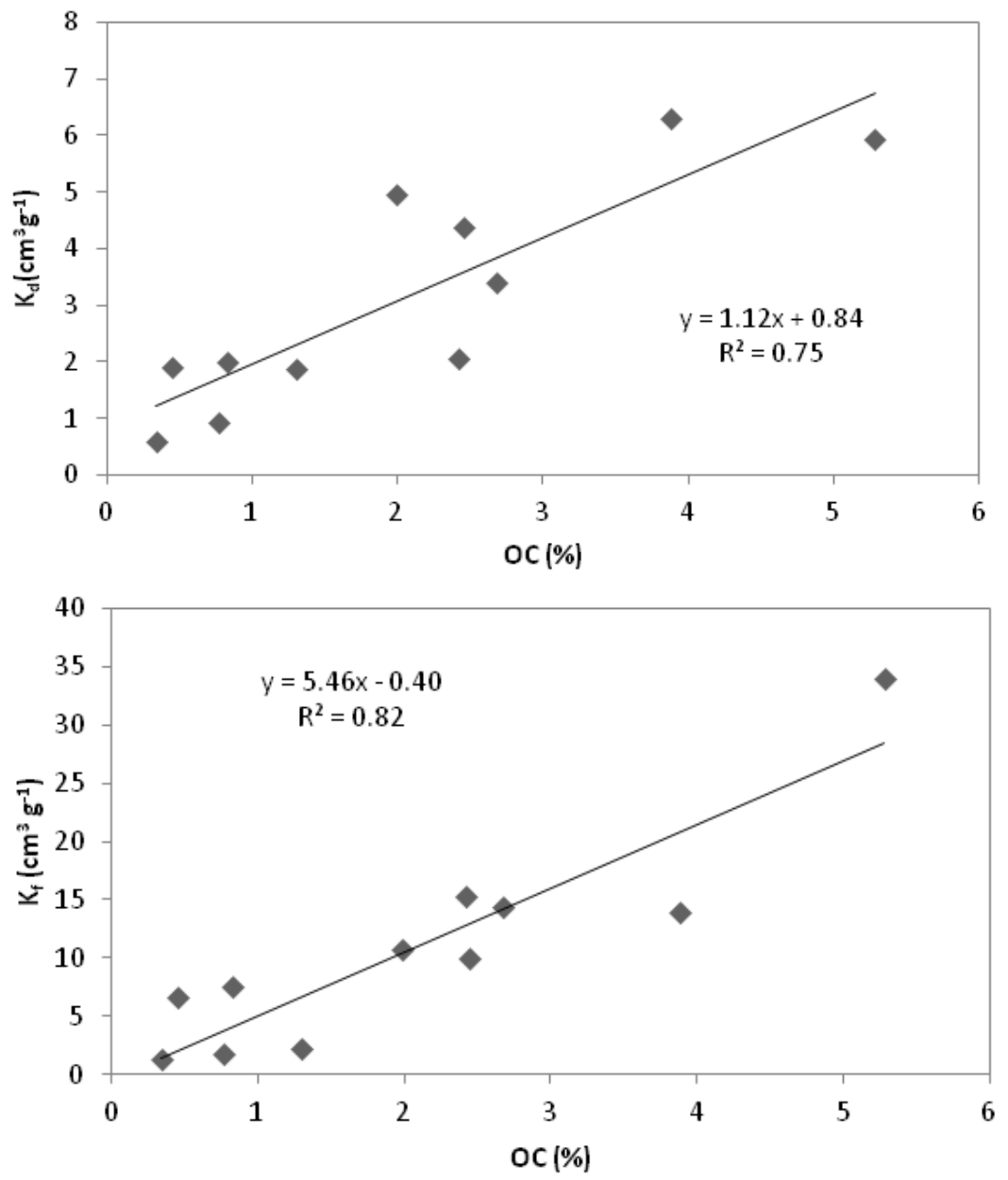

\subsubsection{NTO transport studies}

For a subset of the eight soils used in the batch experiments, we conducted column experiments using NTO. All experiments were conducted in duplicate and replicated well. Figures 40 through 43 show breakthrough curves for Camp Swift, Camp Guernsey, Sassafras, and Arnold AFB soils. The majority of the soils behaved as expected based on the batch studies. For example, the early breakthrough curves and steady-state concentrations seen in Camp Swift soil (Figure 40) were consistent with low $\mathrm{K}_{\mathrm{d}}$ and k estimated in batch studies (Table 10), while Sassafras breakthrough curves (Figure 42) were consistent with higher $K_{d}$ and $k$ measured in batch studies. 
Figure 40. Breakthrough curves for NTO in Camp Swift soil. The dashed grey vertical line indicates timing of $24 \mathrm{hr}$ flow interruption. The tan vertical line indicates the time when the solution was changed back to a saturating solution to observe the desorption phase of the isotherm.
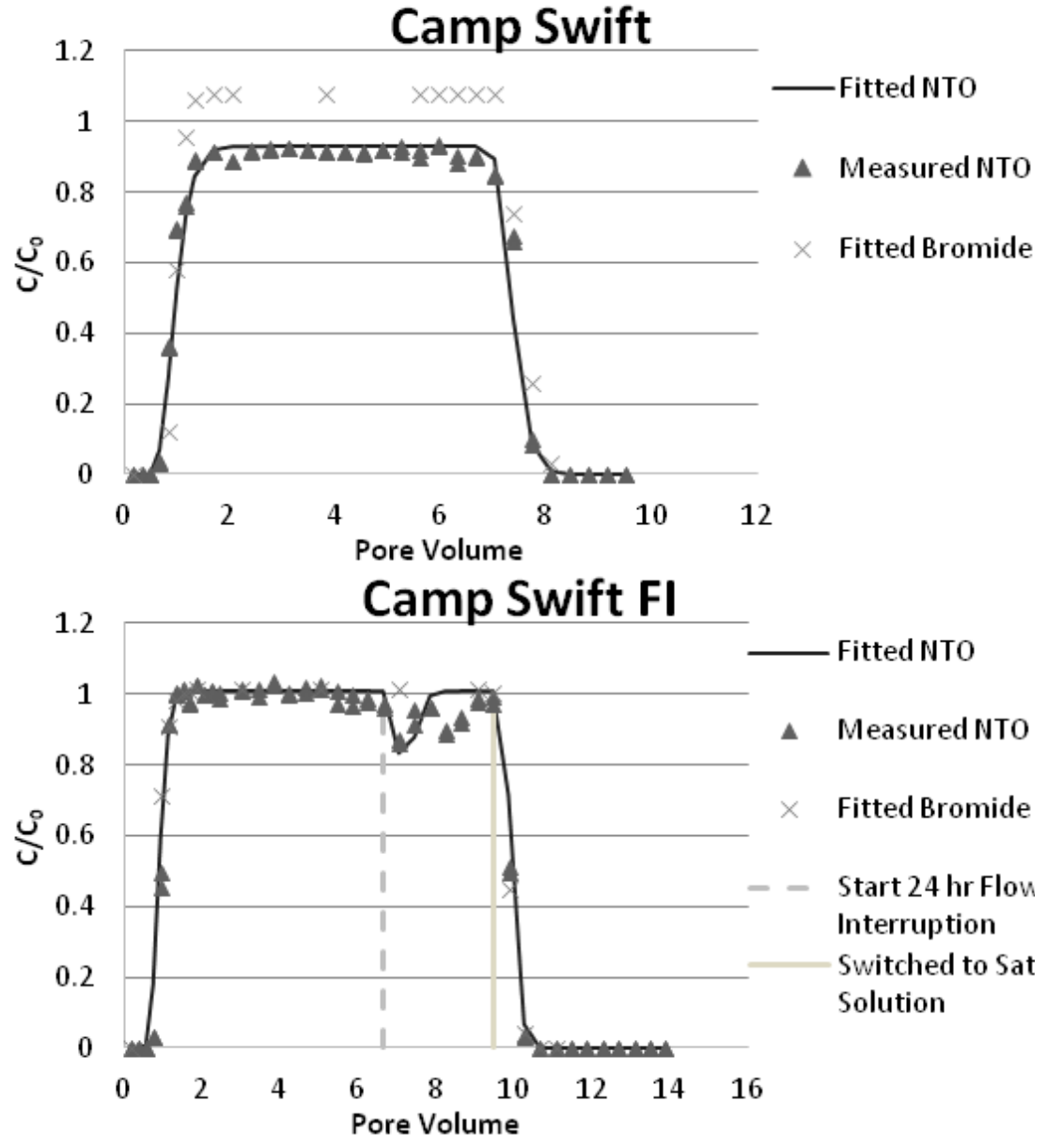
Figure 41. Breakthrough curves for NTO in Camp Guernsey soil. The dashed grey vertical line indicates timing of $24 \mathrm{hr}$ flow interruption. The tan vertical line indicates the time when the solution was changed back to a saturating solution to observe the desorption phase of the isotherm.
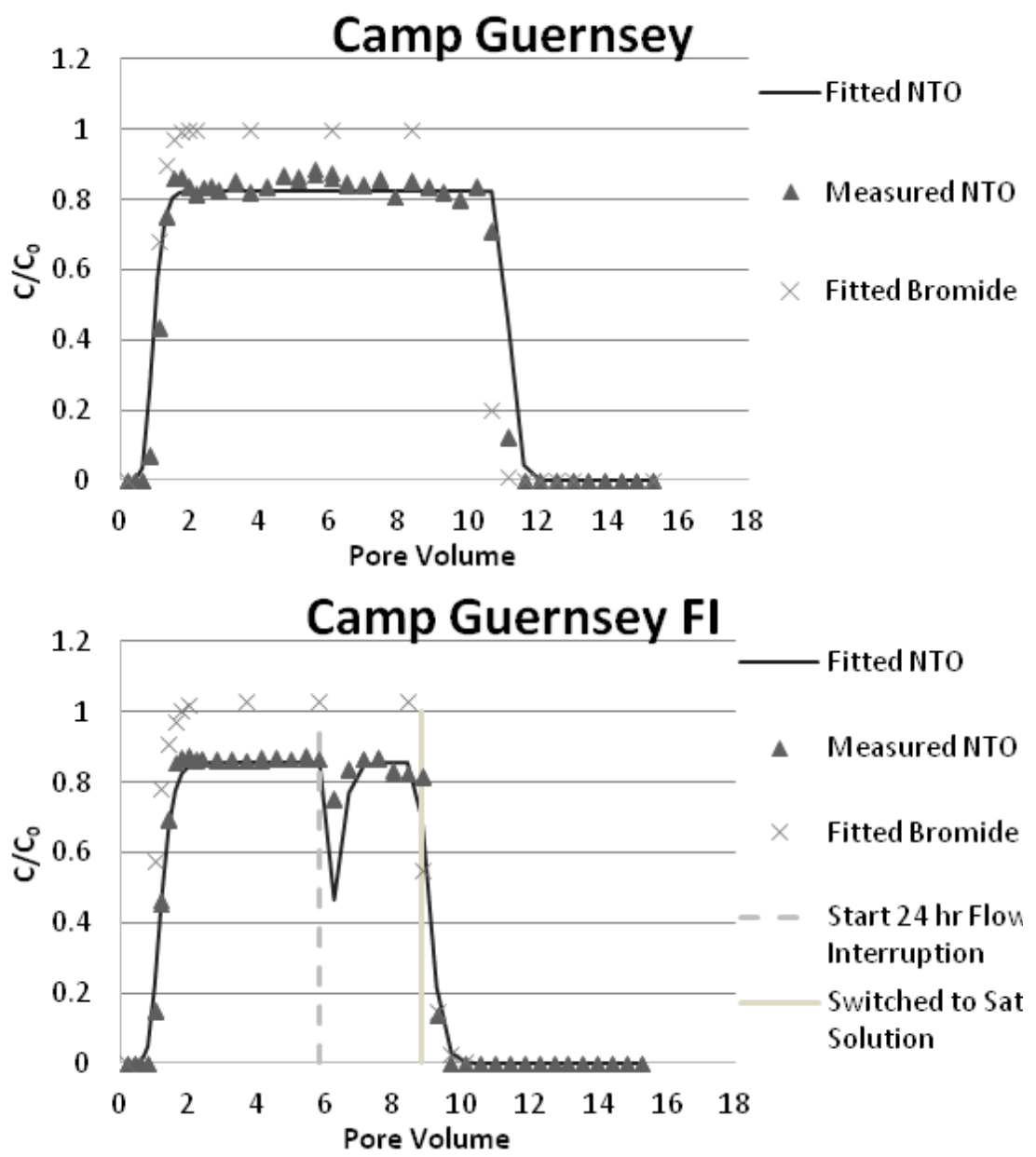
Figure 42. Breakthrough curves for NTO in Sassafras soil. The dashed grey vertical line indicates timing of $24 \mathrm{hr}$ flow interruption. The tan vertical line indicates the time when the solution was changed back to a saturating solution to observe the desorption phase of the isotherm.
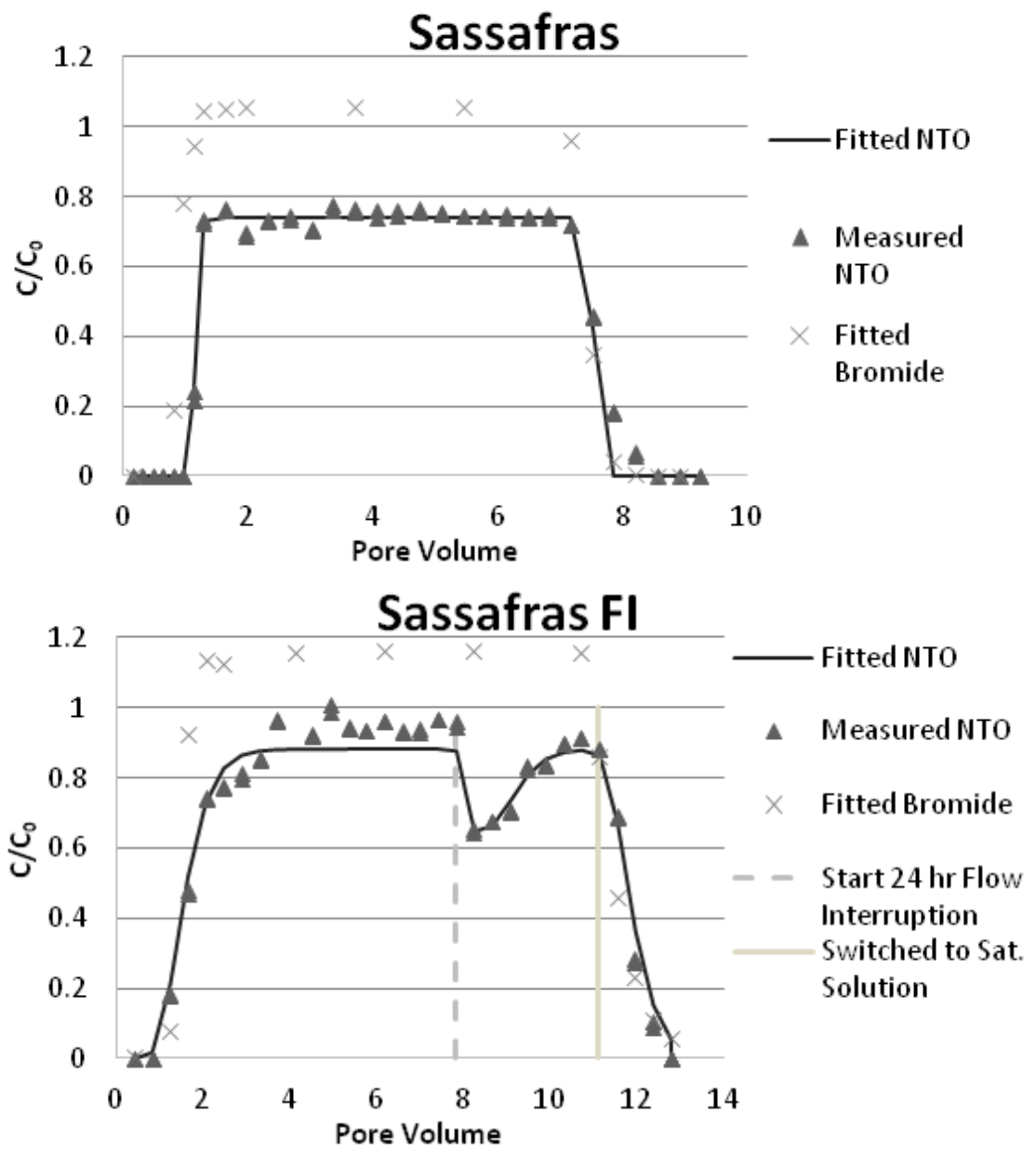
However, several soils, Arnold AFB (Figure 43), Catlin, and Limestone Hills had breakthrough curves that showed increases in transformation rate with time and no recovery after the interrupted flow. Both trends indicate that the mechanism of transformation may be changing as the column becomes increasingly anaerobic with time.

Figure 43. Breakthrough curves for NTO in Arnold AFB soil. The dashed grey vertical line indicates timing of $24 \mathrm{hr}$ flow interruption. The tan vertical line indicates the time when the solution was changed back to a saturating solution to observe the desorption phase of the isotherm.
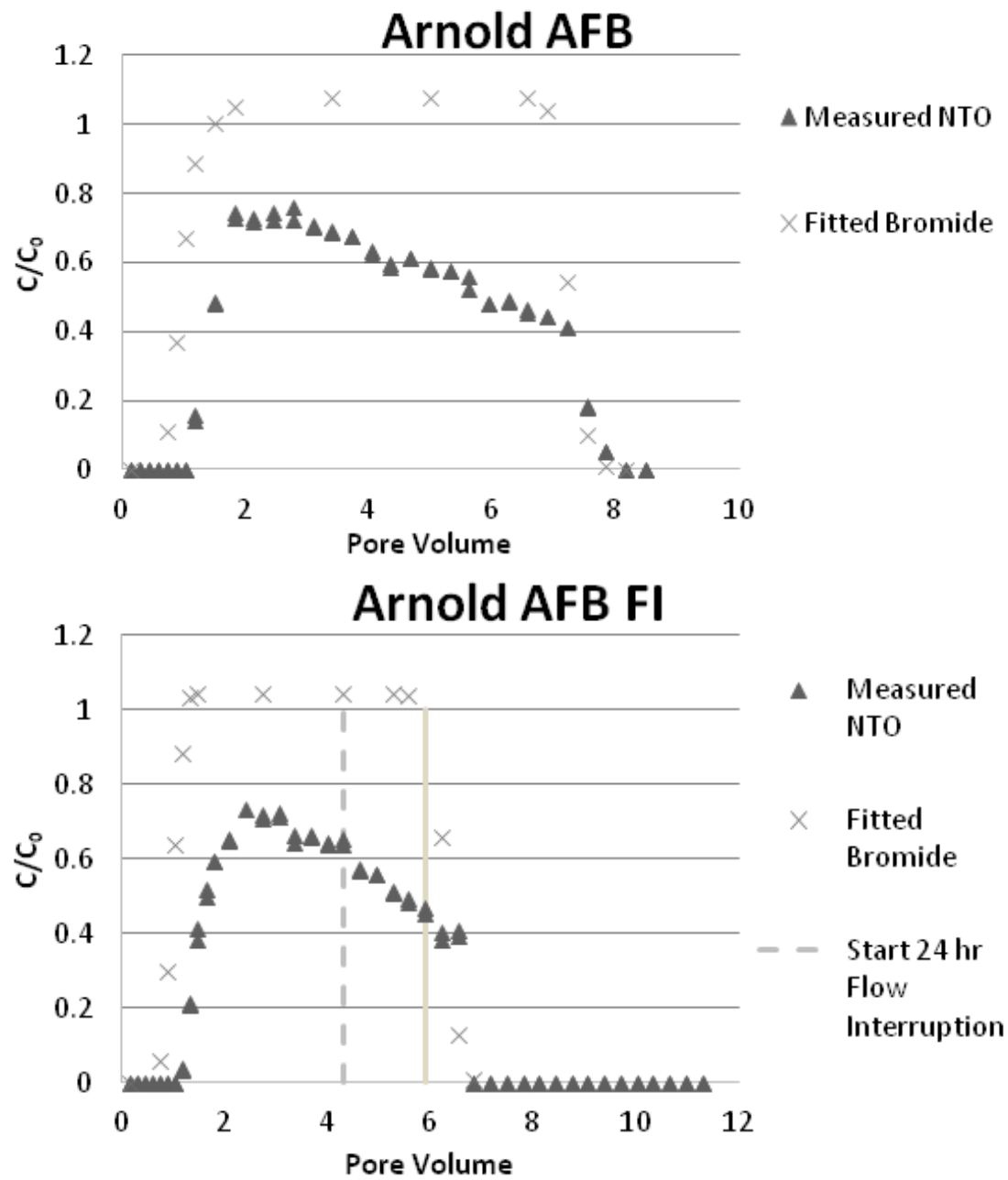

Table 12 presents transport parameters determined from batch and column experiments. As Table 12 shows, a first-order transformation rate constant, $\mathrm{k}$, and values estimated using batch and column experiments were significantly different from each other for all soils that were used in both types of experiments. Their 95\% confidence intervals did not overlap. 
Table 12. Estimates of fate and transport parameters; linear adsorption coefficients, $K_{d, \text { and }}$ transformation rate constants, $k$, for NTO determined from batch and column experiments.

$\mathrm{CE}=95 \%$ confidence interval.

\begin{tabular}{|c|c|c|c|c|c|c|c|c|c|c|c|c|}
\hline \multirow[b]{3}{*}{ Soil } & \multicolumn{6}{|c|}{ Batch estimates } & \multicolumn{6}{|c|}{ Column estimates } \\
\hline & \multicolumn{3}{|c|}{$\mathrm{K}_{\mathrm{d}}\left(\mathrm{cm}^{3} \mathrm{~g}^{-1}\right)$} & \multicolumn{3}{|c|}{$k\left(h^{-1}\right)$} & \multicolumn{3}{|c|}{$\mathrm{K}_{\mathrm{d}}\left(\mathrm{cm}^{3} \mathrm{~g}^{-1}\right)$} & \multicolumn{3}{|c|}{$k\left(h^{-1}\right)$} \\
\hline & Estimate & $\begin{array}{l}\text { Lower } \\
\text { CE }\end{array}$ & $\begin{array}{c}\text { Upper } \\
\text { CE }\end{array}$ & Estimate & $\begin{array}{l}\text { Lower } \\
\text { CE }\end{array}$ & $\begin{array}{l}\text { Upper } \\
\text { CE }\end{array}$ & Estimate & $\begin{array}{l}\text { Lower } \\
\text { CE }\end{array}$ & $\begin{array}{c}\text { Upper } \\
\text { CE }\end{array}$ & Estimate & $\begin{array}{c}\text { Lower } \\
\text { CE }\end{array}$ & $\begin{array}{l}\text { Upper } \\
\text { CE }\end{array}$ \\
\hline Catlin & 0.21 & 0.18 & 0.24 & 0.0221 & 0.0113 & 0.0330 & 0.46 & 0.37 & 0.56 & NA & NA & NA \\
\hline Arnold AFB & 0.34 & 0.29 & 0.38 & 0.0044 & 0.0032 & 0.0055 & 0.83 & 0.36 & 1.29 & NA & NA & NA \\
\hline Camp Butner & 0.10 & 0.07 & 0.14 & 0.0021 & 0.0018 & 0.0024 & 0.42 & 0.20 & 0.64 & 0.185 & 0.114 & 0.255 \\
\hline $\begin{array}{l}\text { Limestone } \\
\text { Hills }\end{array}$ & 0.21 & 0.17 & 0.26 & 0.0123 & 0.0023 & 0.0223 & 0.21 & 0.13 & 0.30 & NA & $\mathrm{NA}$ & NA \\
\hline Sassafras & 0.46 & 0.43 & 0.50 & 0.0080 & 0.0076 & 0.0085 & 0.86 & 0.47 & 1.24 & 0.107 & 0.091 & 0.123 \\
\hline $\begin{array}{l}\text { Camp } \\
\text { Guernsey }\end{array}$ & 0.02 & 0.01 & 0.04 & 0.0004 & 0.0002 & 0.0006 & 0.17 & 0.13 & 0.20 & 0.066 & 0.060 & 0.072 \\
\hline Florence MR & 0.05 & 0.03 & 0.08 & 0.0005 & 0.0004 & 0.0007 & 0.21 & 0.13 & 0.28 & 0.069 & 0.067 & 0.072 \\
\hline Camp Swift & 0.04 & 0.01 & 0.06 & 0.0009 & 0.0007 & 0.0012 & 0.05 & -0.01 & 0.12 & 0.003 & -0.001 & 0.007 \\
\hline
\end{tabular}

Correlation between batch and column NTO linear adsorption coefficients $\left(\mathrm{K}_{\mathrm{d}}\right)$ was better (Figure 44) $(\mathrm{P}=0.00185$, or highly significant). However, the slope of the regression was higher than 1, indicating that there was a trend towards higher $K_{d}$ estimates in the column experiments. Confidence interval overlapped for only two soils, Limestone Hills and Camp Swift, indicating that estimates were different at 95\%.

Figure 44. Correlation between batch and column NTO linear adsorption coefficients $\left(K_{d}\right)$. $\mathrm{P}=0.00185$ for $K_{d}$, which is highly significant.

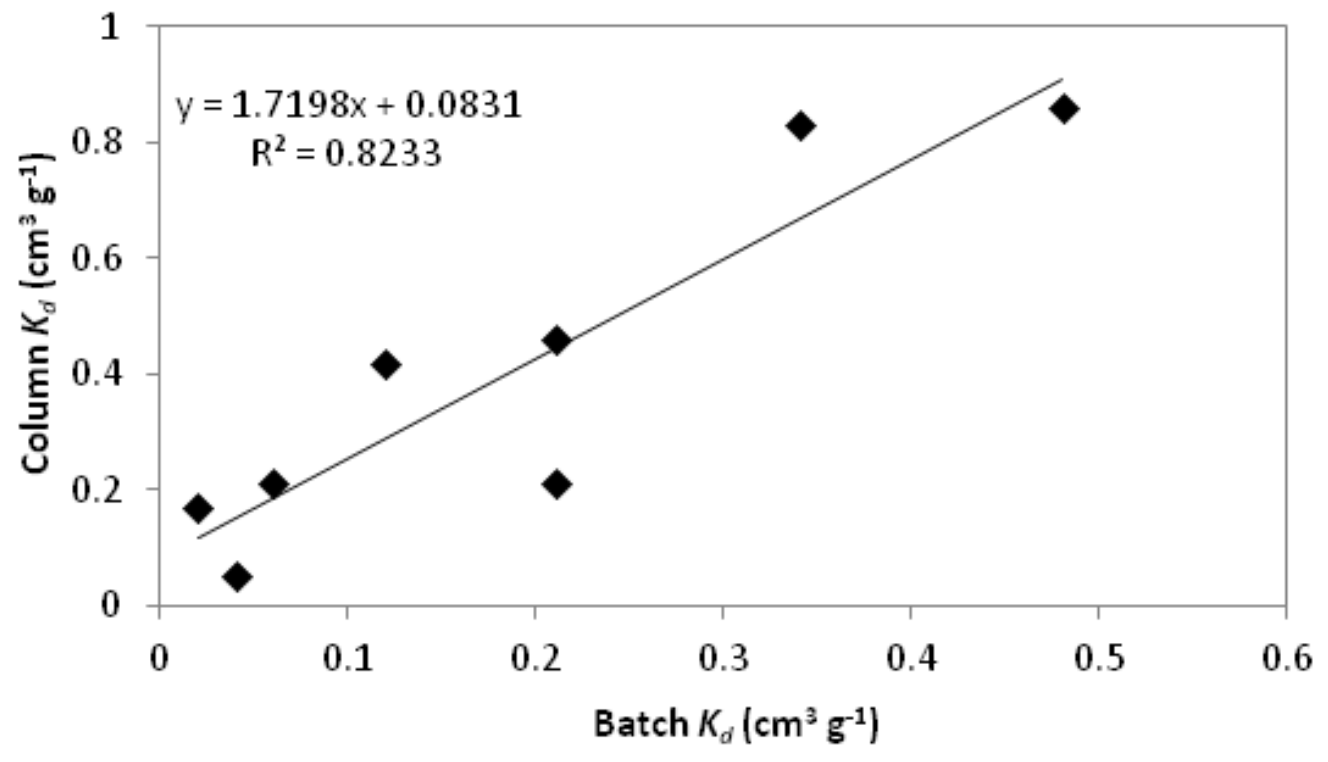




\subsubsection{IMX101 dissolution and transport}

We performed IMX101 dissolution and transport studies using Camp Swift and Camp Guernsey soils that were used earlier in the batch experiments with NTO and DNAN. Both soils were also part of the NTO column studies, and we selected them for the dissolution and transport studies because of their low OM content as DNAN adsorption was expected to be the timelimiting step in the experiment. Soils most significantly differed in their particle size. Camp Guernsey is a sandy soil while Camp Swift has high clay content (Table 9).

IMX101 contains 43.5\% DNAN, 36.8\% NQ, and 19.7\% NTO. Breakthrough curves were observed for all IMX components. In addition, we detected MENA, a DNAN transformation product, in the column outflow. MENA concentrations in the effluent were more than 100 times smaller than measured DNAN concentrations. We did not observe MENA in any of the previous experiments with DNAN conducted in this study. However, the presence of MENA is consistent with the much higher concentrations of parent compound, DNAN, observed in IMX101 column effluents.

Behavior of IM constituents NTO, DNAN, and NQ was consistent with previously known (for NQ) and determined (for NTO and DNAN) fate and transport parameters and agreed with patterns of IMX dissolution observed in drip studies (Figures 45 and 46). NTO was the first compound to break through. NTO also had the highest observed outflow concentrations for all experiments despite representing only about $20 \%$ of the total IMX101 mass. High observed concentrations agree with the high solubility of NTO and with observations in drip studies. Early NTO breakthrough is consistent with low measured adsorption coefficients for both studied soils (0.02 and $0.04 \mathrm{~cm}^{3} \mathrm{~g}^{-1}$ for Camp Guernsey and Camp Swift, respectively). Nitroguanidine, similarly to NTO is a relatively inert compound in soil environments. Nitroguanidine $K_{d}$ values for Sassafras, Plymouth, and Catlin soils were $0.60,0.44$, and $0.24 \mathrm{~cm}^{3} \mathrm{~g}^{-1}$ (Taylor et al., 2012) compared to $0.48,0.50$, and $0.21 \mathrm{~cm}^{3} \mathrm{~g}^{-1}$ measured for NTO in the same soils. Breakthrough curves, however, indicated a slightly later arrival of NQ compared to NTO and a delay in the desorption phase, indicating that NQ has a higher affinity for studied soils than does NTO. Peak concentrations of NQ were lower than those of NTO despite being present at higher concentrations in solid IMX101. The solubility of NTO $\left(16,642 \mathrm{mg} \mathrm{L}^{-1}\right)$ is about four times higher than that of NQ (3800 $\left.\mathrm{mg} \mathrm{L}^{-1}\right)$. 
Figure 45. Breakthrough curves for NTO; NQ; DNAN; and DNAN transformation product, MENA, from IMX101 particle dissolution in Camp Swift soil: (a) undetonated particles and (b) residues of low order detonation. The flow rate is $0.01 \mathrm{~mL} \mathrm{~min}^{-1}$. The black vertical line marks the time when the IMX101 particles were removed from the soil surface to observe desorption phase of the breakthrough.

(a) Camp Swift, detonated IMX 101, column 1

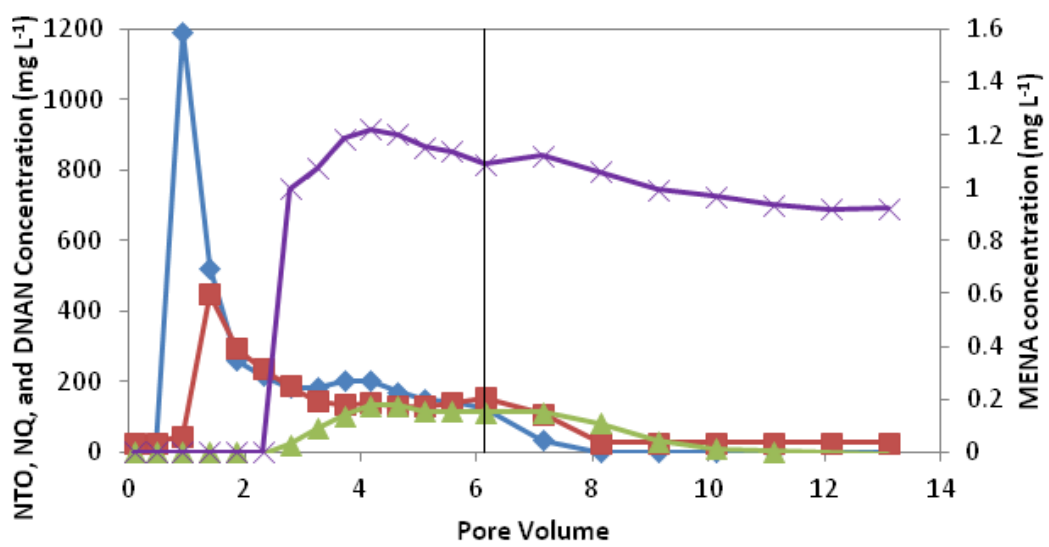

(b)

Camp Swift, undetonated IMX 101, column 3

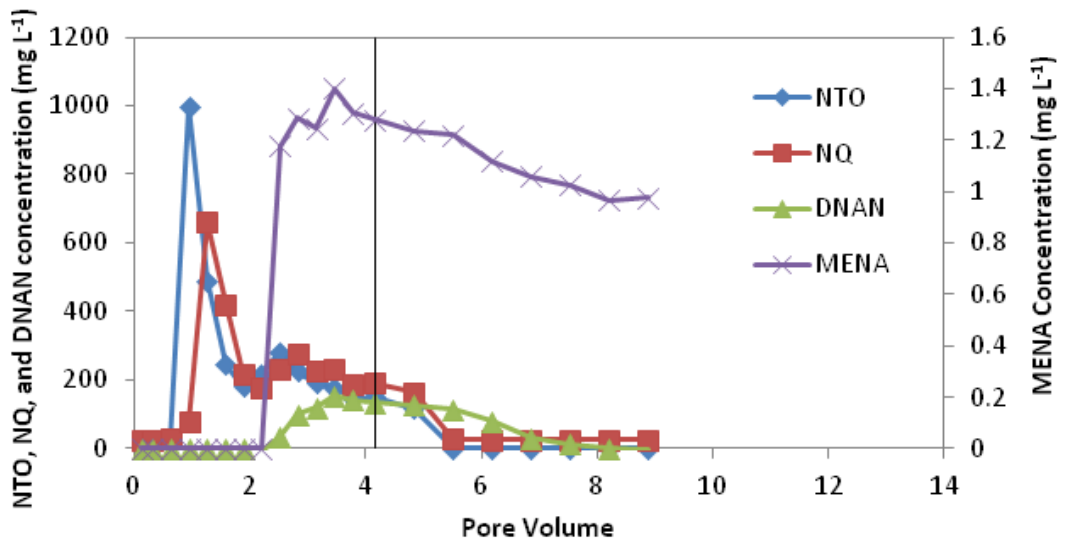


Figure 46. Breakthrough curves for NTO; NQ; DNAN; and DNAN transformation product, MENA, from undetonated IMX101 particle dissolution in Camp Guernsey soil. Flow rate is $0.01 \mathrm{~mL} \mathrm{~min}^{-1}$. The black vertical line marks the time when the IMX101 particle was removed from the soil surface to observe the desorption phase of the breakthrough.

Camp Guernsey, undetonated IMX 101, column 7

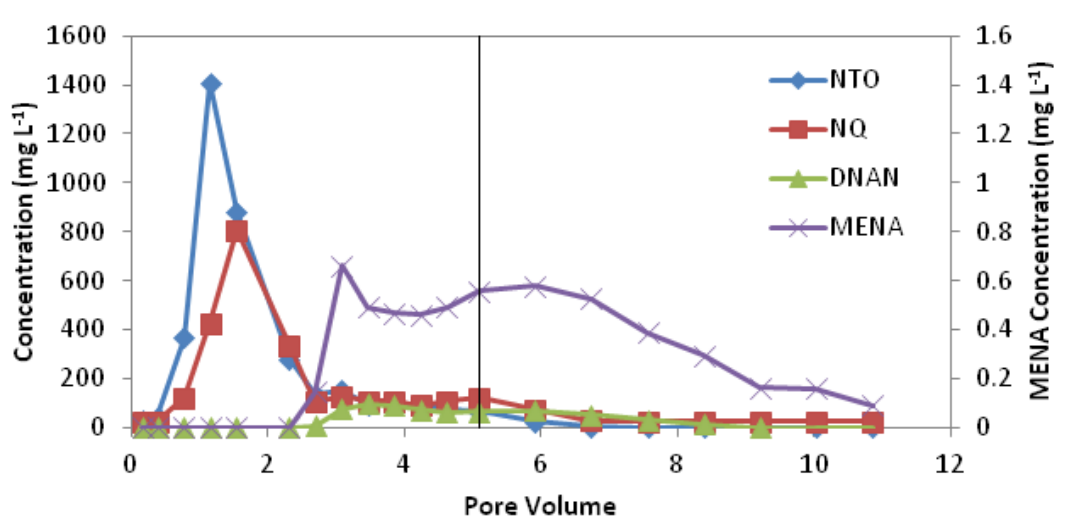

DNAN was the last IM constituent to breakthrough. It also had corresponding delay in the desorption phase of the experiment. In addition, DNAN exhibited the lowest solution concentrations among IM constituents present in IMX101. MENA appeared in the outflow at about the same time as DNAN for Camp Swift soil. However, in Camp Guernsey soil it appeared earlier. If we assume that transformation of DNAN to MENA is occuring in solution, we can conclude that MENA is adsorbing to soils less than DNAN. There was also a difference between adsorption of MENA in Camp Swift and Camp Guernsey soils. MENA concentrations remained high at the end of the experiment in Camp Swift but started to descrease in Camp Guernsey soil. MENA's lower affinity to Camp Guernsey soil as compared to DNAN exhibited itself in a higher sensitivity of MENA to changes in IMX101 dissolution, such as greater roughness of the breakthrough curve and sharper peaks.

We added approximately the same amount of IMX101( $0.05 \mathrm{~g})$ to each column. IMX101 in columns 3, 4, 7, and 8 for the set of experiments performed at $0.01 \mathrm{~mL} \mathrm{~min}^{-1}$ was undetonated while particles used in columns $1,2,5$, and 6 came from low-order detonations. Differences in column outflow between undetonated IMX particles and residues of low-order IMX101 detonations (Figure 45) were within particle-to-particle variability for undetonated IMX101. 


\section{Summary}

The solubility of the individual constituents of IM formulations differ by orders of magnitude, producing porous particles as they dissolve, which increases both the surface areas and the dissolution rates of the particles. The drip dissolution tests and the $\mu \mathrm{CT}$ data show that the components in these explosive formulations dissolve sequentially and in the order predicted by their solubility. The good mass balances measured for the drip tests indicate that the dissolved formulations do not significantly photo- or bio-transform under laboratory conditions. We think that phototransformation is occurring on our outdoor samples because (1) the IM pieces changed color, (2) an unknown peak occurs in the HPLC where polar substances elute, and (3) this peak is largest in samples collected during the summer months when sun irradiance is highest. We expect the dissolved contaminant mixtures and the low $\mathrm{pH}$ of water with NTO to affect microbial communities in ways that are currently unknown. The solubility of most IM constituents is higher than that of TNT and RDX, increasing the likelihood that they will reach groundwater.

We showed that both NTO and DNAN phototransform when in solution. In pure water, DNAN phototransformed more than NTO. However, rates were sensitive to solution chemistry. NTO phototransformation was enhanced in acidic and basic solutions. NTO phototransformation rates also increased three times in the presence of $\mathrm{OM}$, which is common in soil solutions and in surface waters. DNAN was not affected by OM. We found the phototransformation of NTO to be relatively insensitive to temperature between $35^{\circ} \mathrm{C}$ to $70^{\circ} \mathrm{C}$. On the other hand, phototransformation rates of DNAN increased with increasing temperature.

Both NTO and DNAN experienced adsorption and transformation in soils. NTO was weakly adsorbed, with adsorption coefficients lower than those measured for RDX, the explosive compound it is replacing. DNAN, on the other hand, was more strongly adsorbed than TNT. NTO adsorption was not influenced by $\mathrm{OC}$ in the soil but was strongly affected by soil $\mathrm{pH}$. Adsorption decreased as soil $\mathrm{pH}$ increased, probably due to changes in protonation (and therefore charge) of both the soil surfaces and NTO. DNAN adsorption positively correlated with OC while NTO transformation rates increased with increases in soil OC. Column studies supported batch- 
determined parameters but indicated that an increase in transformation rates may be happening in the soil if the soil becomes slightly anaerobic. NTO had little affinity for soils, making it more mobile in the environment than DNAN; and as it is also more soluble, we can expect it to reach ground water faster. DNAN is easily phototransformed and adsorbed in the soil, making it less mobile in the environment. 


\section{References}

ASTM International. 2005. Standard Test Method for Conducting Aqueous Direct Photolysis Tests. ASTM E 896-92. West Conshohocken, PA: ASTM International.

ASTM International. 2008. Standard Test Method for Determining a Sorption Constant $\left(\mathrm{K}_{\mathrm{oc}}\right)$ for an Organic Chemical in Soil and Sediments. ASTM E 1195-01. West Conshohocken, PA: ASTM International.

Boddu, V. M., K. Abburi, S. W. Maloney, and R. Damavarapu. 2008. Thermophysical properties of an insensitive munitions compound, 2,4-dinitroanisole. J ournal of Chemical and Engineering Data 53:1120- 1125.

Boparai, H. K., S. D. Comfort, T. Satapanajaru, J . E. Szecsody, P. R. Grossl, P. J . Shea. 2010. Abiotic transformation of high explosives by freshly precipitated iron minerals in aqueous Feıı solutions. Chemosphere 79 (8): 865- 872.

Bordeleau, G., R. Martel, G. Ampleman, and S. Thiboutot. 2013. Photolysis of RDX and nitroglycerin in the context of military training ranges. Chemosphere 93:14- 19.

Brannon, J. M., and J . C. Pennington. 2002. Environmental Fate and Transport Process Descriptors for Explosives. ERDC/ EL TR-02-10. Vicksburg, MS: U.S. Army Engineer Research and Development Center,.

Brunauer, S., P. H. Emmett, and E. Teller. 1938. Adsorption of gases in multimolecular layers. J ournal of the American Chemical Society 60:309-319.

Brusseau, M. L., Q. Hu, and R. Srivastava. 1997. Using flow interruption to identify factors causing nonideal contaminant transport. J ournal of Contaminant Hydrology 24:205- 219.

Brusseau, M. L., P. S. C. Rao, R. E. J essup, and J . M. Davidson. 1989. Flow Interruption: A method for investigating sorption nonequilibrium. J ournal of Contaminant Hydrology 4:223-240.

Chakka, S., V. M. Boddu, S. W. Maloney, R. Damavarapu. 2010. Prediction of Physicochemical Properties of Energetic Materials via EPI Suite. In Energetic Materials, ed. V. Boddu and P. Redner, 77-92. New York, NY: CRC Press.

Chipen, G. I., R. P. Bokalder, and V. Y. Grinstein. 1966. 1,2,4-Triazol-3-one and its nitro and amino derivatives. Chemistry of Heterocyclic Compounds 2:110- 116.

Coppola, E. N. 2007. Treatment technologies for perchlorate. Presented at the Global Demil Symposium, 17 May. http://www.dtic.mil/ndia/2007global_demil/SessionIVA/ 0850Coppola.pdf.

Davies, P. J ., and A. Provatas. 2006. Characterisation of 2,4-Dinitroanisole: An Ingredient for Use in Low Sensitivity Melt Cast Formulations. Weapons Systems Division, Defence Science and Technology Organisation. 
Dodard, S. G., M. Sarrazin, J . Hawari, L. Paquet, G. Ampleman, S. Thiboutot, and G. I. Sunahara. 2013. Ecotoxicological assessment of a high energetic and insensitive munitions compound: 2,4-Dinitroanisole (DNAN). J ournal of Hazardous Materials 262:143- 150.

Dontsova, K. M., S. L. Yost, J . Šimůnek, J . C. Pennington, and C. W. Williford. 2006. Dissolution and Transport of TNT, RDX, and Composition B in Saturated Soil Columns. J ournal of Environmental Quality 35:2043-2054.

Dontsova, K. M., J . C. Pennington, S. Yost, and C. Hayes. 2007. Transport of nitroglycerin, nitroguanidine and diphenylamine in soils. In Characterization and Fate of Gun and Rocket Propellant Residues on Testing and Training Ranges: Interim Report 1. ERDC TR-07-1. Vicksburg, MS: U.S. Army Engineer Research and Development Center,.

Dontsova, K. M., M. Chappell, J . Šimůnek, and J . C. Pennington. 2008. Dissolution and transport of nitroglycerin, nitroguanidine and ethyl centralite from M9 and M30 propellants in soils. In Characterization and Fate of Gun and Rocket Propellant Residues on Testing and Training Ranges: Final Report. ERDC TR-08-1. Vicksburg, MS: U.S. Army Engineer Research and Development Center.

Dontsova, K. M., C. Hayes, J . C. Pennington, and B. Porter. 2009a. Sorption of high explosives to water-dispersible clay: Influence of organic carbon, aluminosilicate clay, and extractable iron. J ournal of Environmental Quality 38:1458- 1465.

Dontsova, K. M., C. Hayes, J . Šimůnek, J . C. Pennington, and C. W. Williford. 2009b. Dissolution and transport of 2,4-DNT and 2,6-DNT from M1 propellant in soil. Chemosphere 77:597-603.

Dumitras-Hutanu, C. A., A. Pui, S. J urcoane, E. Rusu, and G. Drochioiu. 2009. Biological effect and the toxicity mechanisms of some dinitrophenyl ethers. Romanian Biotechnological Letters 14:4893-4899.

Fung, V., D. Price, E. LeClaire, J . Morris, N. Tucker, and A. Carrillo. 2010. Further development and optimization of IM ingredients at Holston Army Ammunition Plant. In 2010 Insensitive Munitions and Energetic Materials Technology Symposium, 11- 14 October, Munich, Germany.

Haderlein, S. B., K. W. Weissmahr, and R. P. Schwarzenbach. 1996. Specific adsorption of nitroaromatic explosives and pesticides to clay minerals. Environmental Science and Technology 30:612-622.

Hawari, J ., R. Saad, N. Perreault, Z. Radovic, A. Halasz, L. Paquet, D. Manno, G. I. Sunahara, S. Dodard, M. Sarrazin, S. Thiboutot, G. Ampleman, S. Brochu, E. Diaz, A. Gagnon, and A. Marois. 2011. Environmental fate and ecological impact of NTO, DNAN, NQ, FOX-7, and FOX-12 considered as substitutes in the formulations of less sensitive composite explosives. Biotechnology Research Institute of the National Research Council. 
Hawari, J ., N. Perreault, A. Halasz, L. Paquet, Z. Radovic, D. Manno, G. I. Sunahara, S. Dodard, M. Sarrazin, S. Thiboutot, G. Ampleman, S. Brochu, E. Diaz, A. Gagnon, and A. Marois. 2012. Environmental Fate and Ecological Impact of NTO, DNAN, NQ, FOX-7, and FOX-12 Considered as Substitutes in the Formulations of Less Sensitive Composite Explosives. Ottawa, Onario: National Research Council Canada.

Kofman, T. P., M. S. Pevzner, L. N. Zhukova, T. A. Kravchenko, and G. M. Frolova. 1980. Methylation of 3-Nitro-1,2,4-Triazol-5-one. Zhurnal organicheskoi khimii 16:420- 425 .

Le Campion, L., M. T. Adeline, and J . Ouazzani. 1997. Separation of NTO related 1,2,4triazole-3-one derivatives by a high performance liquid chromatography and capillary electrophoresis. Propellants, Explosives, Pyrotechnics 22:233- 237.

Le Campion, L., M. Delaforge, J . P. Noel, and J . Ouazzani. 1998. Metabolism of C-14labelled 5-nitro-1,2,4-triazol-3-one (NTO): comparison between rat liver microsomes and bacterial metabolic pathways. J ournal of Molecular Catalysis BEnzymatic 5:395- 402.

Le Campion, L., C. Giannotti, and J . Ouazzani. 1999a. Photocatalytic degradation of 5nitro-1,2,4-triazol-3-one NTO in aqueous suspention of TiO2. Comparison with Fenton oxidation. Chemosphere 38:1561- 1570.

Le Campion, L., A. Vandais, and J . Ouazzani. 1999b. Microbial remediation of NTO in aqueous industrial wastes. Fems Microbiology Letters 176:197- 203.

Lever, J . H., S. Taylor, L. Perovich, K. Bjella, and B. Packer. 2005. Dissolution of Composition B detonation residuals. Environmental Science and Technology 39:8803- 8811.

London, J . O., and D. M. Smith. 1985. A toxicological study of NTO. Los Alamos National Laboratory Report.

McDonald, L. M., J r., and V. P. Evangelou. 1997. Optimal solid-to-solution ratios for organic chemical sorption experiments. Soil Science Society of America J ournal 61:1655- 1659.

O’Sullivan, D. W., J . R. Denzel, and D. J . Luning Prak. 2011. Photolysis of 2,4,6Trinitrotoluene in Seawater: Effect of Salinity and Nitrate Concentration. In Environmental Chemistry of Explosives and Propellant Compounds in Soils and Marine Systems: Distributed Source Characterization and Remedial Technologies, 157- 169. American Chemical Society.

Olivares, C., J . Liang, L. Abrell, R. Sierra-Alvarez, and J . A. Field. 2013. Pathways of reductive 2,4-dinitroanisole (DNAN) biotransformation in sludge. Biotechnology and Bioengineering 110:1595- 1604.

Pelletier, P., D. Lavigne, I. Laroche, F. Cantin, L. Phillips, and V. Fung. 2010. Additional properties studies of DNAN based melt-pour explosive formulations. In 2010 Insensitive Munitions and Energetic Materials Technology Symposium, 11- 14 October, Munich, Germany. 
Perreault, N. N., D. Manno, A. Halasz, S. Thiboutot, G. Ampleman, and J . Hawari. 2011. Aerobic biotransformation of 2,4-dinitroanisole in soil and soil Bacillus sp. Biodegradation 23 (2): 287- 295.

Prak, D.L., and D. O’Sullivan. 2012. Photochemical Transformation of Munitions Constituents in Marine Waters. United States Naval Academy.

Rao, B., W. Wang, Q. Cai, T. Anderson, and B. Gu. 2013. Photochemical transformation of the insensitive munitions compound 2,4-dinitroanisole. Science of the Total Environment 443:692-699.

Rasband, W. S. 2012. ImageJ . Bethesda, MD: U. S. National Institutes of Health. http://imagej.nih.gov/ij/.

Rhoades, J . D., N. A. Manteghi, P. J . Shouse, and W. J . Alves. 1989. Estimating Soil Salinity from Saturated Soil-Paste Electrical Conductivity. Soil Science Society of America J ournal 53:428-433.

Roy, W. R., I. G. Krapac, S. F. J . Chou, and R. A. Griffin. 1992. Batch-Type Procedures for Estimating Soil Adsorption of Chemicals. Cincinnati, OH: U.S. Environmental Protection Agency, Risk Reduction Engineering Laboratory.

Rutkowski, J ., R. Cirincione, and C. Patel. 2010. Common low-cost insensitive munitions explosive to replace TNT and Comp B. In 2010 Insensitive Munitions and Energetic Materials Technology Symposium, 11- 14 October, Munich, Germany.

Smith, M. W., and M. D. Cliff. 1999. NTO based explosive formulations: A technology Review. Aeronautical and Maritime Research Laboratory, Weapons Systems Division.

Sokkalingam, N., J . J . Potoff, V. M. Boddu, and S. W. Maloney. 2008. Prediction of Environmental Impact of High-Energy Materials with Atomistic Computer Simulations. Detroit, MI: Wayne State University, Department of Chemical Engineering. http://www.dtic.mil/cgi-bin/GetTRDoc?Location=U2\&doc=GetTRDoc.pdf\&AD $=$ ADA503638.

Spear, R. J ., C. N. Louey, and M. G. Wolfson. 1989. A Preliminary Assessment of 3-Nitro1,2,4-Triazol-5-One (NTO) as an Insensitive High Explosive. Maribyrnong, Australia: Defense Science and Technology Organization Materials Research Laboratory.

Taylor, S., J . H. Lever, J . Fadden, N. Perron, and B. Packer. 2009a. Outdoor weathering and dissolution of TNT and Tritonal. Chemosphere 77:1338- 1345.

Taylor, S., J . H. Lever, J . Fadden, N. Perron, and B. Packer. 2009b. Simulated rainfalldriven dissolution of TNT, Tritonal, Comp B and Octol particles. Chemosphere 75:1074- 1081.

Taylor, S., J . H. Lever, M. E. Walsh, J . Fadden, N. Perron, S. Bigl, R. Spanggord, M. Curnow, and B. Packer. 2010. Dissolution rate, weathering mechanics and friability of TNT, Comp B, Tritonal, and Octol. ERDC/ CRREL TR-10-2. Hanover, NH: U.S. Army Engineer Research and Development Center 
Taylor, S., K. Dontsova, S. Bigl, C. Richardson, J . Lever, J . Pitt, J .P Bradley, M. Walsh, and J. Šimůnek. 2012. Dissolution Rate of Propellant Energetics from Nitrocellulose Matrices. ERDC/ CRREL TR-12-9. Hanover, NH: U.S. Army Engineer Research and Development Center.

Taylor S., D. B. Ringelberg, K. Dontsova, C. Daghlian, M. E. Walsh, and M. R. Walsh 2013. Insights into the dissolution and the three-dimensional structure of insensitive munitions formulations. Chemosphere 93:1782- 1788.

Taylor, S., E. Park, K. Bullion, and K. Dontsova. 2015. Dissolution of three insensitive munitions formulations. Chemosphere 119:342- 348.

Thorn, K. A., and K. R. Kennedy. 2002. ${ }^{15} \mathrm{~N}$ NMR investigation of the covalent binding of reduced TNT amines to soil humic acid, model compounds, and lignocellulose. Environmental Science and Technology 36:3878-3796.

Thorn, K. A., J . C. Pennington, and C. A. Hayes. 2001. Transformation of TNT in an aerobic compost: Structure and reactivity effects in the covalent binding of aromatic amines to organic matter. Abstracts of Papers of the American Chemical Society 41:628-632.

Thorn, K. A., J . C. Pennington, K. R. Kennedy, L. G. Cox, C. A. Hayes, and B. E. Porter. 2008. N-15 NMR study of the immobilization of 2,4- and 2,6-dinitrotoluene in aerobic compost. Environmental Science and Technology 42:2542- 2550.

Tucker, W. A., G. J. Murphy, and E. D. Arenberg. 2002. Adsorption of RDX to soil with low organic carbon: Laboratory results, field observations, remedial implications. Soil and Sediment Contamination 11:809-826.

U.S. Environmental Protection Agency (U.S. EPA). 1998. Indirect Photolysis Screening Test. In Fate, Transport and Transformation Test Guidelines. OPPTS 835.5270 (EPA-712-C-98-099). Washington, DC: U.S. Environmental Protection Agency.

U.S. Environmental Protection Agency (U.S. EPA). 2006. Nitroaromatics, nitramines and nitrate esters by high performance liquid chromatography. Method 8330B. In Test Methods for Evaluating Solid Waste, Physical/ Chemical Methods. EPA SW846. Washington, DC: U.S. Environmental Protection Agency. http://www.epa.gov/osw/hazard/testmethods/pdfs/8330b.pdf.

U.S. Environmental Protection Agency (U.S. EPA). 2010. Standard Operating Procedure for Analysis of Total Organic Carbon in Sediments (Dry Combustion, IR Detection), Revision 04. In Sampling and Analytical Procedures for GLNPO's Open Lake Water Quality Survey of the Great Lakes. EPA 905-R-05-001. Chicago, IL: U.S. Environmental Protection Agency, Great Lakes National Program Office

Uchimiya, M., L. Gorb, O. Isayev, M. M. Qasim, and J. Leszczynski. 2010. One-electron standard reduction potentials of nitroaromatic and cyclic nitramine explosives. Environmental Pollution 158:3048-3053.

Walsh, M. R., M. E. Walsh, S. Taylor, C. A. Ramsey, D. B. Ringelberg, J . E Zufelt, S. Thiboutot, G. Ampleman, and E. Diaz. 2013. Characterization of PAX-21 insensitive munitions detonation residues. Propellants, Energetics, Pyrotechnics 38:399- 409. 
Walsh, M. R., M. E. Walsh, C. A. Ramsey, S. Thiboutot, G. Ampleman, E. Diaz, J. E. Zufelt. 2014. Energetic Residues from the Detonation of IMX-104 Insensitive Munitions. Propellants, Energetics, Pyrotechnics 39:243- 250.

Wilson, A. 2007. Explosive Ingredients and Compositions for the IM M795 Artillery Ammunition. Presented at the 2007 Insensitive Munitions and Energetic Materials Technology Symposium, 15-18 October, Miami, FL. http://www.dtic.mil/ndia/2007im_em/BBriefs/7Wilson.pdf.

Yoon, J . M., D. J . Oliver, and J . V. Shanks. 2005. Plant Transformation Pathways of Energetic Materials (RDX, TNT, DNTs). In Agricultural Biotechnology: Beyond Food and Energy to Health and the Environment, ed. A. Eaglesham, R. Bessin, R. Trigiano, R. W. T. Hardy, 103- 116. National Agricultural Biotechnology Council Report 17. Ithaca, New York: National Agricultural Biotechnology Council. 


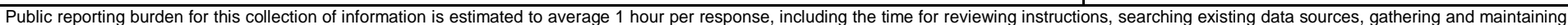

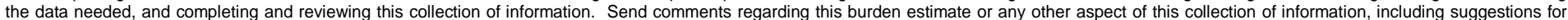

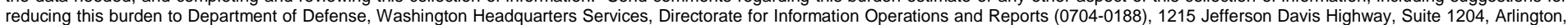

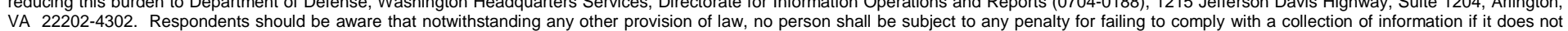
display a currently valid OMB control number. PLEASE DO NOT RETURN YOUR FORM TO THE ABOVE ADDRESS.

\begin{tabular}{l|l}
$\begin{array}{l}\text { 1. REPORT DATE (DD-MM-YYYY) } \\
09-29-2013\end{array}$ & $\begin{array}{l}\text { 2. REPORT TYPE } \\
\text { Technical Report/Final }\end{array}$ \\
\hline
\end{tabular}

4. TITLE AND SUBTITLE

Dissolution of NTO, DNAN, and Insensitive Munitions Formulations and Their Fates in Soils

3. DATES COVERED (From - To)

5a. CONTRACT NUMBER

5b. GRANT NUMBER

5c. PROGRAM ELEMENT NUMBER

5d. PROJECT NUMBER

5e. TASK NUMBER

5f. WORK UNIT NUMBER

8. PERFORMING ORGANIZATION REPORT NUMBER

ERDC/CRREL TR-14-23

Cold Regions Research and Engineering Laboratory (CRREL)

U.S. Army Engineer Research and Development Center (ERDC)

72 Lyme Road

Hanover, NH 03755-1290

9. SPONSORING I MONITORING AGENCY NAME(S) AND ADDRESS(ES)

Strategic Environmental Research and Development Program (SERDP)

Alexandria, VA 22350-3605

Katerina Dontsova, Susan Taylor, Rose Pesce-Rodriguez, Mark Brusseau, Jennifer Arthur, Noah Mark, Marianne Walsh, James Lever, and Jiri Šimůnek

7. PERFORMING ORGANIZATION NAME(S) AND ADDRESS(ES)

12. DISTRIBUTION / AVAILABILITY STATEMENT

Approved for public release; distribution is unlimited.

\section{SUPPLEMENTARY NOTES}

Strategic Environmental Research and Development Program (SERDP)

\section{ABSTRACT}

New explosive compounds that are less sensitive to shock and high temperatures are being tested as replacements for TNT and RDX. Two of these explosives, DNAN (2,4-dinitroanisole) and NTO (3-nitro-1,2,4-triazol-5-one), have good detonation characteristics and are the main ingredients in a suite of insensitive munitions (IM) explosives that are being, or soon will be, fielded. Both compounds, however, are more soluble than either TNT or RDX; and research has shown that both have some human and environmental toxicity. Data on their fate is needed to determine if DNAN and NTO have the potential to reach groundwater and be transported off base, an outcome that could create future contamination problems on military training ranges and trigger regulatory action. In this study, we measured how quickly DNAN and NTO dissolve in IM formulations and how solutions of these IM explosives interact with different types of soils. Because both dissolution and solution-soil interactions are determined by a suite of parameters, we are using a multifaceted approach to studying these processes. Given a mass of IM compounds scattered on the range, our work will help determine the dissolved IM masses, their subsequent transport and fate, and their likelihood of reaching groundwater.

\section{SUBJECT TERMS}

Dissolution

DNAN (2,4-dinitroanisole)

16. SECURITY CLASSIFICATION OF:

\section{a. REPORT}

Unclassified b. ABSTRACT

Unclassified
IM Solution-soil interactions

Insensitive Munitions (IM)

SERDP

SERDP

c. THIS PAGE

Unclassified

\begin{tabular}{|c|c|}
\hline $\begin{array}{l}\text { 17. LIMITATION } \\
\text { OF ABSTRACT }\end{array}$ & $\begin{array}{l}\text { 18. NUMBER } \\
\text { OF PAGES }\end{array}$ \\
\hline SAR & 92 \\
\hline
\end{tabular}

19a. NAME OF RESPONSIBLE PERSON

19b. TELEPHONE NUMBER (include area code) 